\title{
An expanded toolkit for Drosophila gene tagging using synthesized homology donor constructs for CRISPR mediated homologous recombination
}

Oguz Kanca ${ }^{1,2}$, Jonathan Zirin ${ }^{3}$, Yanhui Hu${ }^{3}$, Burak Tepe ${ }^{1,2}$, Debdeep Dutta ${ }^{1,2}$, WenWen Lin ${ }^{1,2}$, Liwen Ma ${ }^{1,2}$, Ming Ge $e^{1,2}$, Zhongyuan Zuo ${ }^{1,2}$, Lu-Ping Liu ${ }^{3}$, Robert W. Levis $^{4}$, Norbert Perrimon ${ }^{3,6}$, Hugo J. Bellen ${ }^{1,5}$

1) Department of Molecular and Human Genetics, Baylor College of Medicine, Houston, TX

2) Duncan Neurological Research Institute, Texas Children Hospital, Houston, TX

3) Department of Genetics Harvard Medical School, Boston, MA

4) Department of Embryology and Howard Hughes Medical Institute, Carnegie Institution for Science, Baltimore, MD

5) Department of Neuroscience, Baylor College of Medicine, Houston, TX

6) Howard Hughes Medical Institute, Harvard Medical School, Boston, MA

\section{Abstract}

Previously, we described a large collection of Drosophila strains that each carry an artificial exon containing a T2AGAL4 cassette inserted in an intron of a target gene based on CRISPR-mediated homologous recombination (Lee et al., 2018). These alleles permit numerous applications and have proven to be very useful. Initially, the homologous recombination-based donor constructs had long homology arms $(>500$ bps) to promote precise integration of large constructs ( $>5 \mathrm{~kb})$. Recently, we showed that in vivo linearization of the donor constructs enables insertion of large artificial exons in introns using short homology arms (100-200 bps) (Kanca et al., 2019a). Shorter homology arms make it feasible to commercially synthesize homology donors and minimize the cloning steps for donor construct generation. Unfortunately, about $50 \%$ of Drosophila genes lack suitable coding introns for integration of artificial exons. Here, we report the development of new set of constructs that allow the replacement of the coding region of genes that lack suitable introns with a KozakGAL4 cassette, generating a knock-out/knock-in allele that expresses GAL4 similarly as the targeted gene. We also developed custom vector backbones to 
further facilitate and improve transgenesis. Synthesis of homology donor constructs in custom plasmid backbones that contain the target gene sgRNA obviates the need to inject a separate sgRNA plasmid and significantly increases the transgenesis efficiency. These upgrades will enable the targeting of nearly every fly gene, regardless of exon-intron structure, with a $70-80 \%$ success rate.

\section{Introduction}

The Drosophila Gene Disruption Project (GDP) aims to generate versatile genetic tools for most genes to facilitate the study of gene function in vivo and to create fly stocks for the community. The CRISPR mediated integration cassette (CRIMIC) approach is a recent addition to the GDP to target fly genes. The CRIMIC strategy is based on integrating a Swappable Integration Cassette (SIC) containing an artificial exon encoding attP-FRT-Splice Acceptor (SA)-T2AGAL4-polyA-3XP3EGFP-polyA$F R T$-attP (T2AGAL4). The SIC is integrated in an intron between two coding exons (coding intron) by CRISPR mediated homologous recombination (Lee et al., 2018; Gnerer et al., 2015; Diao et al., 2015). The viral T2A sequence leads to the truncation of the nascent target gene polypeptide and re-initiation of translation of the downstream GAL4 as an independent protein. This cassette typically creates a strong loss of function allele of the targeted gene and expresses the yeast GAL4 transcription factor in a similar spatial and temporal pattern as the protein encoded by the targeted gene (Lee et al., 2018). These alleles can be used to: 1) determine the gene expression pattern; 2) study the effect of loss of function of the gene product; 3) replace the SIC through Recombinase Mediated Cassette Exchange (RMCE) (Bateman et al., 2006; Venken et al., 2011) with an artificial coding exon that encodes a fluorescent protein to assess protein subcellular localization (Venken et al., 2011) and identify interacting proteins ; 4) express UAS-cDNAs of the targeted gene and its variants to assess rescue of the mutant phenotype and conduct structure/function studies (Wangler et al., 2017); 5) excise the insert with UASFlippase to revert the phenotype (Lee et al., 2018). 
The introduction of an artificial exon is only feasible for genes that contain a suitable large coding intron, typically $100 \mathrm{nt}$ or more. This requirement makes nearly half of the genes inaccessible to strategies based on the use of artificial exons (Supplementary table 1; Figure 1A). In addition, the genes that do not have a suitable intron are typically smaller in size than genes that contain a suitable intron, and usually have fewer previously isolated, publicly available alleles than larger genes with a suitable intron.

Here, we describe the development of a knock-out/knock-in strategy to replace the coding sequence of genes with a Kozak sequence-GAL4 polyA-FRT-3XP3GFPpolyA-FRT (KozakGAL4) cassette to target genes that lack introns that are suitable for artificial exon knock-ins. The targeted gene is cut by Cas9 using two sgRNAs, one targeting the 5' untranslated region (UTR) and the other the 3' UTR. We targeted $\sim 100$ genes with this strategy and show that about $80 \%$ of the integrated KozakGAL4 cassettes lead to UAS-mCherry expression in the $3^{\text {rd }}$ instar larval brain, a ratio that is similar to what was observed for the T2AGAL4 strategy (Lee et al., 2018).

We also improved the design of the homology donor constructs that can be used for integration of either KozakGAL4 or T2AGAL4 cassettes. The use of short homology arms allows commercial DNA synthesis of the entire gene-specific portion of the donor plasmid, a cheaper and more efficient option than PCR-amplification and cloning of each homology arm (Kanca et al., 2019a). To further extend this approach, we developed a method in which the DNA sequences directing the transcription of the target gene-specific $\operatorname{sgRNA}(\mathrm{s})$ are synthesized together in the same segment as the homology arms. The design allows gene specific sgRNA to be synthesized together with the homology arms, eliminating the need to clone and inject a separate vector for the sgRNAs. We tested our new designs on $\sim 200$ genes and show that the upgrades result in a transgenesis efficacy of $\sim 80 \%$. The strategies that we introduce here allow targeting of nearly every gene in the fly genome, further streamline the generation of homology donor DNAs, increase efficiency as compared to previous strategies, and improve the rate of precise genome editing. 


\section{Results and Discussion}

\section{The KozakGAL4 cassette}

Integration of an artificial exon is only feasible for genes that have coding introns large enough to identify an sgRNA target site that is located at a sufficient distance from the preceding splice donor and the following splice acceptor site. Based on our experience, an intron should be larger than $100 \mathrm{nt}$ to be suitable for integration of an artificial exon. An analysis of the Drosophila genome shows that 5787 out of 13,931 protein-coding genes have a sufficiently large coding intron that is shared among all the annotated splicing isoforms of the gene. However, 8144 genes lack such introns, making them inaccessible for the T2AGAL4 and other artificial exon-based strategies. Genes with a suitable intron typically have larger coding sequences (2051 nt vs $1321 \mathrm{nt}$ ) and have a larger number of previously isolated mutant alleles based on FlyBase data (10.4 vs 3.4) than genes without a suitable intron(s) (Figure 1A, Supplementary table 1; Larkin et al., 2020). To integrate a GAL4 cassette that can be used in a similar manner as the T2AGAL4 insertions, we developed the KozakGAL4 knock-in/knock-out strategy. Kozak sequence is an optimal translation initiation site in eukaryotic mRNAs, and it is identified as (C/A) AA (C/A) AUG in Drosophila (Cavener, 1987; Kozak, 1986). We use CAAA as a Kozak sequence upstream of the start codon of GAL4. To replace the coding region of genes, we typically identify sgRNA target sites in the 5' UTR and 3' UTR (Figure 1B). To retain possible gene expression regulation by the 5'UTR, we select the upstream sgRNA target site that is closest to the start codon and that is not predicted to have off-target activity based on CRISPR Optimal Target Finder (Gratz et al., 2014). The location of the downstream sgRNA target site in the 3'UTR is less stringent since the endogenous 3'UTR is not included in the final transcript due to the polyA signal in the KozakGAL4 cassette. The median 5' and 3' UTR lengths for Drosophila genes are 214 and 224 bps respectively which are typically large enough to identify putative sgRNA targets (Chen et al., 2011; Jan et al., 2011). In our experience the 5'UTR typically contains multiple sgRNA targets whereas 3'UTRs contain few candidate sgRNA target sites due to their $\mathrm{A} / \mathrm{T}$ rich nature. In cases where a suitable sgRNA target site cannot be found in the 3'UTR, we target a site within the coding region, close to the stop codon, to minimize the coding region of the gene that remains. In cases where a suitable sgRNA site cannot be found within the 5'UTR region, the 
search is expanded to the promoter region and the sequence between the gRNA cut site and transcription start site is added to the homology region. In such cases, a single nucleotide substitution to eliminate the PAM sequence is introduced in the homology donor construct, preventing cutting of the homology donor.

We also developed alternative strategies to target genes without suitable introns and for which no proper sgRNA site could be identified within the 5'UTR or promoter. We generated the SA-KozakGAL4-polyA-3XP3EGFP-polyA cassette that can be introduced in an intron within the 5'UTR (Figure 1 Supplementary Figure1A). Alternatively, for genes with small coding introns, we make two cuts: one within a coding intron just upstream of the SA of an exon; the second in the 3'UTR. The excised sequence is then replaced with a T2AGAL4 cassette (Figure 1 Supplementary Figure 1B).

\section{KozakGAL4 alleles drive expression of UAS-transgenes}

There are two main approaches to generate alleles that express GAL4 that are not based on the T2AGAL4-based strategies. The first one is based on an insertion of minimal promoter-GAL4 coding sequences in a transposon backbone. The strategy is called enhancer trapping and was based originally on GFP and LacZ rather than GAL4 (O'Kane and Gehring, 1987; Bellen et al., 1989). Upon mobilization of the transposon, lines are established where the GAL4 expression pattern is of interest (Brand and Perrimon, 1993; Lukacsovich et al., 2001; Hacker et al., 2003; Gohl et al., 2011). Given that they are inserted in the genome by transposable elements they are not always optimally placed to report the full expression pattern of the gene (Spradling et al., 1995; Mayer et al., 2013) but they have been used extensively as many reflect the expression pattern of a nearby gene (Wilson et al., 1989). Many however are not mutagenic (Spradling et al., 1999). The second strategy to generate alleles that may express GAL4 in the expression pattern of a gene is to clone a 500 bps-5kb region upstream of the promoter of the gene upstream of the GAL4 coding sequences and inserting the transgene in the genome. There are large collections of these enhancer-GAL4 alleles and most aim to report the expression of enhancer fragments rather than reporting the expression pattern of the gene from which they are derived (Jenett et al., 2012; Manning et al., 2012; Pfeiffer et al., 
2008). Hence, the available approaches can now be complemented with the KozakGAL4 approach that should incorporate all or most upstream regulatory information and generate a null allele of the targeted gene. The latter greatly facilitates rescue experiments using UAS-cDNA transgenes.

To assess the KozakGAL4 strategy, we targeted 109 genes to date and successfully replaced the coding region of 82 genes with the KozakGAL4 cassette (Supplemental Table 2). We crossed 57 of these with UAS-CD8mCherry transgenic flies to determine the GAL4 expression of the targeted gene in the brain of wandering third instar larvae. Our previous findings, using T2AGAL4 alleles have shown that $\sim 80 \%$ of all T2AGAL4 alleles lead to specific expression in third instar larval brains (Lee et al., 2018). Similarly, with KozakGAL4 alleles, we detected specific GAL4 expression for about $80 \%(46 / 57)$ of the genes (Figure 2A). Although KozakGAL4 targeted genes are typically small, limiting the possible regulatory information in the coding region, it is possible that excision of coding and some UTR sequences may remove part of the regulatory input. We therefore tested whether a few KozakGAL4 alleles drive the expression of the UAS-CD8mCherry in a similar pattern as the targeted gene. We selected a KozakGAL4 allele that drives expression of the reporter in a restricted group of cells in the $3^{\text {rd }}$ instar larval brain and analyzed the single cell RNA sequencing (scRNAseq) data for the $3^{\text {rd }}$ instar larval brain to determine cell clusters that express the targeted gene (Ravenscroft et al., 2020). We then used the same scRNAseq dataset to determine other genes expressed in overlapping clusters and that we previously targeted with T2AGAL4. KozakGAL4 driven UAS-CD8mCherry reporter is expressed in a very similar expression pattern compared to T2AGAL4 driven reporter expression of the genes that we identified through scRNAseq. The other genes expressed in the overlapping cluster according to scRNAseq, such as serp, verm and emp suggest that this cluster corresponds to tracheal cells, which is in line with the observed expression pattern through imaging (Figure 2B, Figure 2 Supplementary Figure 1B; Luschnig et al., 2006; Lee et al., 2018). Comparison of the expression patterns of the other tested KozakGAL4 targeted genes and T2AGAL4 targeted genes that are expressed in overlapping cell groups showed overlapping expression patterns based on imaging as well (Figure 2 Supplementary Figure 1A). Hence, the use of scRNAseq data can provide an independent means of verification of accuracy of the observed reporter expression patterns. 
Next, we determined if UAS-human or fly cDNAs could rescue the phenotype associated with 11 gene deletions caused by the KozakGAL4 knock-ins. For pngl, Wdr37, Tom70, CG8320, CG16787 and IntS11 a UAS-fly or human cDNA rescued the KozakGAL4 induced phenotypes, suggesting that the KozakGAL4 is expressed where the targeted gene product is required for the gene function. For pex2, pex16, fitm, PIG-A and CG34293 the expression of orthologous human cDNA did not rescue the associated phenotypes.

In summary, KozakGAL4 offers a new means to disrupt gene function while expressing the GAL4 in the expression domain of the targeted genes. This approach allows us to tag the remainder of the genes that do not contain a suitable coding intron for the T2AGAL4 strategy which corresponds to $58 \%$ of all the genes.

\section{New vector backbones for synthesis of homology donor constructs that are also templates for sgRNA expression}

We previously showed that linearizing the homology donor constructs in vivo allows for integration of large constructs in the genome through CRISPR-mediated homologous recombination even using short homology arms (Kanca et al., 2019a).

This approach makes inexpensive commercial synthesis of homology donor intermediates feasible. The intermediate vectors can be used for a single step directional cloning of the SIC in the homology donor intermediate vector. This greatly facilitates the generation of homology donor vectors which previously required fourway ligations with large homology arms. Moreover, this eliminates cloning failures ( 20-30\%) and troubleshooting associated constructs with large homology arms (Kanca et al., 2019a). The resulting new homology donor vectors were previously injected together with two vectors that express two sgRNAs (pCFD3, Port et al., 2014) in embryos that express Cas9 in their germline. The first sgRNA targets the homology donor vector backbone to linearize the homology donor and does not have a target in the Drosophila genome (sgRNA1, Garcia-Marques et al., 2019). The second sgRNA vector expresses the sgRNA to target the gene and introduce the double strand DNA break that serves as a substrate for homologous recombination. In Kanca et al. (2019a) we demonstrated that injection of these constructs resulted in transgenesis efficiencies of about $60 \%$. 
We developed new approaches to increase the transgenesis efficiency of the custom DNA backbones, decrease the workload, and to simplify the generation of homology donor constructs. The first custom vector backbone that we tested has the U6$3:: s g R N A 1$ sequence in the vector backbone and sgRNA1 targets, on either side of the EcoRV site where the synthesized fragments are directionally integrated (vector backbone named pUC57_Kan_gw_OK, design named int200, Figure 3A). With this design, the homology donor vector intermediates that are commercially synthesized contain the sgRNA1 coding sequence, obviating the need to co-inject one of the sgRNA vectors. Having the sgRNA1 coding region in the backbone also helps with in vivo linearization of the homology donor since the homology donor construct and the sgRNA1 are delivered together in a single vector. The int200 design also removes the sgRNA1 target sites from the synthesized region as they are present in the vector. This allows increasing the homology arm length to 200 bps without increasing the cost of synthesis.

We tested this int200 design for 397 genes with T2AGAL4 cassettes and 36 genes with KozakGAL4 cassette. For each construct, we injected 400-600 embryos that express Cas 9 in the germline. For inserting the KozakGAL4 cassette, the two gene specific sgRNAs were cloned into pCFD5 (Port and Bullock, 2016). For both T2AGAL4 and KozakGAL4 insertions the int200 homology donor plasmid was coinjected with the plasmid that encodes the target specific sgRNA (pCFD3 for the former and pCFD5 for the latter case). We successfully integrated T2AGAL4 cassette in 252 genes ( $65 \%$ success rate) and replaced the coding region of 22 genes with KozakGAL4 ( $61 \%$ success rate) (Figure $3 B$ ). PCR verification of the inserts was performed by using gene specific PCR primers outside the homology region pointing towards the insert and a construct specific PCR primer. For $88 \%$ of the T2AGAL4 inserts we obtained PCR verification on both sides of the insert and for the remaining $12 \%$ we obtained $\mathrm{PCR}$ products on one side of the construct. For $91 \%$ of KozakGAL4 inserts we obtained amplicons on both sides of the insert. For the inserts with a single PCR verification, we sequenced the amplicon to ensure the insert is in the proper locus. Hence, the overall transgenesis success rate of the int200 method is about 65\% (Figure 3B). This is very similar to the injection success rate of homologous recombination using large $(0.5-1 \mathrm{~kb})$ homology arms (1165 
insertions in 1784 targeted genes, Lee et al., 2018) but leads to very significant reductions in labor and cost. Additionally, int200 facilitates the cloning of homology donor constructs and eliminates cloning failures which reduce the overall successful targeting rate using large homology arms to $~ 50 \%$ (successful cloning of $80 \%$ constructs that are injected with $65 \%$ transgenesis success rate). In summary, the int200 method provides a $\sim 30 \%$ gain in overall efficiency (from $50 \%$ to $65 \%$ ).

To further optimize the custom vector backbone we repositioned the U6$3::$ sgRNA1and added a partial tRNA construct directly upstream of the EcoRV site that is used to insert the synthesized fragments (Figure 4A). The partial tRNA allows adding the gene-specific sgRNA sequence (vector named pUC57_Kan_gw_OK2 and design named gRNA_int200 for T2AGAL4 constructs and named 2XgRNA_int200 for KozakGAL4 constructs). Hence, two or three sgRNA can be produced from the single injected plasmid. One of the sgRNA1 target sites is added to the synthesized fragment before the start of the homology arm and the other sgRNA1 target site is added to the backbone just downstream of the EcoRV site where the synthesized fragment is directionally inserted. This design obviates the need to clone a separate sgRNA vector to target the genomic locus. It also ensures simultaneous delivery of all the components of the homologous recombination reaction as they are delivered on a single plasmid. We have targeted 127 genes with gRNA_int200_T2AGAL4 donor plasmids (Supplemental Table 2) and successfully inserted the T2AGAL4 cassette in 95 genes ( 75\% success rate, Figure 4B). We also tested whether genes for which the tagging failed using the int200 strategy (Figure 3) could be targeted with the gRNA_int200_T2AGAL4 using the same genespecific sgRNA and homology arms. For 3 out of 4 genes tested, use of the gRNA_int200 strategy resulted in successful integration of T2AGAL4 cassette. These data show that incorporating all the sgRNAs in the donor vector improves the transgenesis efficiency.

For the KozakGAL4 constructs, we inserted a second tRNA sequence after the first synthesized sgRNA and added the second gene specific sgRNA sequence to the synthesis reaction. We targeted 72 genes with 2XgRNA_int200_KozakGAL4 cassette and successfully inserted KozakGAL4 in 59 genes ( 82\% success rate, Figure 4B; Supplemental Table 2). We tested whether genes for which the tagging 
failed using the int200_KozakGAL4 strategy (Figure 3) could be properly targeted with the 2XgRNA_int200_KozakGAL4 using the same gene specific sgRNAs and homology arms sequences and again observed that for 3 out of 4 tested genes, the 2XgRNA_int200_KozakGAL4 strategy was successful. In summary, the gRNA_int200 design increases transgenesis rate and streamlines the creation of T2AGAL4 and KozakGAL4 constructs by obviating the need to generate a separate sgRNA expression plasmid and ensuring co-delivery of all components for homologous recombination. In summary, the gRNA_int200 allows a 78\% transgenesis success rate, or an additional $20 \%$ when compared to the int200 approach (increase from $65 \%$ to $78 \%$ ).

\section{Use of 2XgRNA_int200 intermediate vectors for GFP tagging}

We have previously shown that integrating a SA-linker-EGFP-FIAsH-Strepll$3 x T E V$ cs-3xFLAG-linker-SD (SA-GFP-SD) in coding introns of genes is an efficient approach to tag proteins with GFP (Venken et al., 2011; Nagarkar-Jaiswal et al., 2015a; 2015b; 2017; Li-Kroeger et al., 2018). We typically generate these alleles through Recombinase Mediated Cassette Exchange of existing MiMIC SICs and we have shown that they are functional in $72 \%$ of tested genes (Nagarkar-Jaiswal et al., 2015a). A major factor that affects the functionality of the GFP protein trap is the insertion position. In cases where the artificial exon encoding for protein trap is inserted in a coding intron that bisects a predicted functional protein domain, the resulting protein trap is often not functional. Hence, another efficient approach to tag proteins encoded by genes that have no intron, small introns or no suitable MiMICS in any preselected position in the protein structure is highly desirable.

We tested the use of synthesized homology donor intermediate vectors to replace the coding sequence of genes without suitable coding introns with the gene coding sequence fused to GFP at different locations. We selected the $W d r 37$ gene as it has a small intron (Kanca et al., 2019b). We amplified Wdr37 sequences from the genome by PCR and used NEB HiFi DNA assembly to generate homology donor constructs where a sfGFP tag is integrated at the $\mathrm{N}$ terminus, $\mathrm{C}$ terminus or internally (Figure 5, Figure 5 Supplementary figure 1,2,3 for schematics of HiFi assembly). The 3XP3 DsRed flanked by PiggyBac transposase inverted repeats is integrated after the 3'UTR and serves as the transformation marker that can be 
excised precisely using the PiggyBac transposase (flyCRISPR.molbio.wisc.edu; Bier et al., 2018) (Figure 5A). The assembled sequences are subcloned in the synthesized homology donor intermediate. Injection of the homology donor plasmids in embryos expressing Cas9 in their germline resulted in positive transgenics in each case. Western blot of the resulting protein trap alleles using anti-GFP antibody detected bands at the expected length for the tagged protein in each case. However, the internally tagged allele is less abundant, underlining that the placement of sfGFP tag can affect protein stability (Figure 5B). Hence, the strategy to replace the whole coding region with a GFP tagged coding region allows tagging almost any gene in any position in the coding sequence.

In summary, we developed a KozakGAL4 strategy to target the genes that do not have a suitable intron and a set of novel custom vector backbones to facilitate homology donor construct production and increase transgenesis rate. The methods we developed are versatile and can be modified to generate GAL4 gene traps or GFP protein fusions of the targeted genes. Finally, the methodology we describe should be easy to implement in any other model organisms to facilitate generation of gene trap and protein trap alleles.

\section{Acknowledgements}

We thank the Bloomington Drosophila Stock Center and FlyBase for fly stocks acquisition and distribution and the Developmental Studies Hybridoma Bank for antibodies. We thank Stephanie Mohr for critical reading of the manuscript. We thank Yuchun He, Ying Fang, Minhua Huang, Zhihua Wang, Yaping Yu, Junyan Fang, Ruifang Zhang, and Lily Wang for generating and maintaining MiMIC/CRIMIC T2A-GAL4 fly stocks. Confocal microscopy was performed in the BCM IDDRC Neurovisualization Core, supported by the NICHD (U54HD083092). HJB received support from NIH R01GM067858, R24 OD031447, R24OD022005, U54NS093793 HJB and the Huffington Foundation. OK receive support from NIH R24 OD031447. JZ, YHu, and NP receive support from NIGMS (GM067761 and GM084947). NP is an investigator of the Howard Hughes Medical Institute. RWL was supported by the Carnegie Institution for Science. 


\section{Materials and Methods}

\section{Generation of homology donor constructs}

Templates for ordering the int200 and gRNA_int200 constructs can be found in Supplementary Materials and Methods. Homology donor intermediate vectors were ordered for production from Genewiz ("ValueGene” option) in pUC57 Kan_gw_OK (for int200 strategy) or pUC57 Kan_gw_OK2 (for gRNA_int200 strategy) vector backbone at $4 \mu \mathrm{g}$ production scale. The lyophilized vectors were resuspended in 53 $\mu \mathrm{l}$ of $\mathrm{ddH}_{2} \mathrm{O}$. 1 ul was used for Golden Gate assembly with $290 \mathrm{ng}$ of pM37 vector of reading frame phase corresponding to the targeted intron (for T2GAL4, Lee et al., 2018) or 265 ng of pM37_KozakGAL4 vector (for KozakGAL4). The Golden Gate Assembly reaction was set in 200 $\mu$ l PCR tubes (ThermoScientific AB2000) with 2.5 $\mu$ l 10X T4 DNA ligase buffer (NEB B0202S), $0.5 \mu$ l T4 DNA ligase (NEB M0202L), 1 $\mu$ restriction enzyme (Bbsl_HF or Bsal_HFv2 NEB R3559L and R3733L respectively), $1 \mu \mathrm{l}$ of SIC (pM37_T2AGAL4 or pM37_KozakGAL4 at $290 \mathrm{ng} / \mu \mathrm{l}$ or $265 \mathrm{ng} / \mu \mathrm{l}$ respectively), $19 \mu \mathrm{l}$ of $\mathrm{dH}_{2} \mathrm{O}$ and $1 \mu \mathrm{l}$ of homology donor construct. For cloning multiple constructs in parallel, master mixes were prepared including all the components except for the homology donor intermediate vector. The reactions were incubated in a Thermocycler (cycle 30 times between $37^{\circ} \mathrm{C} 5$ minutes, $16^{\circ} \mathrm{C} 5$ minutes, then $65^{\circ} \mathrm{C} 20$ minutes, $8^{\circ} \mathrm{C}$ hold). An additional digestion step was done to

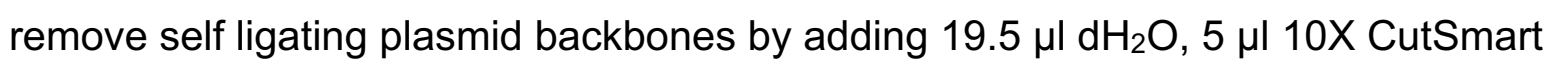
buffer (NEB B7204S) and $0.5 \mu \mathrm{l}$ Bbsl or Bsal_HFv2 (the enzyme used for the cloning reaction). The reaction product was transformed in $\mathrm{DH} 5 \alpha$ competent cells and plated on Kanamycin+ LB plates.

\section{Fly injections}

Int200-T2AGAL4 and int200-KozakGAL4 constructs were injected at $250 \mathrm{ng} / \mu \mathrm{l}$ along with $100 \mathrm{ng} / \mathrm{\mu l}$ gene specific gRNA(s) cloned in pCFD3 or pCFD5 respectively (Port et al. 2014; Port and Bullock, 2016). Injections were performed as described in (Lee et al., 2018). 400-600 embryos from $y^{1} w^{*}$; iso18; attP2 $(y+)\{$ nos-Cas $9(v+)\}$ for genes on the $2^{\text {nd }}$ or $4^{\text {th }}$ chromosome and $y^{1} w^{*}$ iso $6 ;$; attP2 $(y+)$ nnos-Cas $\left.9(v+)\right\}$ for genes on the $\mathrm{X}$ chromosome and $y^{1} w^{*}$; $\operatorname{attP40}\left(y^{+}\right)\{$nos-Cas9 $(v+)\}$; iso5 (Kondo and Ueda, 2013) for genes on the $3^{\text {rd }}$ chromosome per genotype were injected. Whole genome 
sequencing BAM files of isogenized lines can be found at:

https://zenodo.org/record/1341241. Resulting G0 males and females were crossed individually to $y^{1} w^{*}$ flies as single fly crosses for 3XP3-EGFP detection. Positive lines were balanced, and stocks were established. Up to 5 independent lines were generated per construct per gene. The list of generated alleles can be found on Supplementary table 2 . The sequences of homology arms and sgRNA(s) as well as the results of PCR validation and imaging on third instar larval brain are available at http://flypush.imgen.bcm.tmc.edu/pscreen/crimic/crimic.php. The stocks are deposited in the Bloomington Drosophila Stock Center (BDSC) on a regular basis. The stocks are available from the Bellen lab until they are deposited and established in the BDSC.

\section{PCR validation}

PCR primers that flank the integration site were designed for each targeted gene. These primers were used in combination with insert-specific primers that bind 5 ' of the inserted cassette in reverse orientation and 3' of the insert in forward orientation (pointing outwards from the insert cassette, Primer sequences can be found in the supplementary material). 200-800 nt amplicons were amplified from genomic DNA from individual insertion lines through single fly PCR (Gloor et al., 1993) using OneTaq PCR master mix (NEB \#M0271L). PCR conditions were $95^{\circ} \mathrm{C}$ for 30 seconds, $95^{\circ} \mathrm{C} 30$ seconds, $58^{\circ} \mathrm{C} 30$ seconds, $68^{\circ} \mathrm{C} 1$ minute for 34 cycles and $68^{\circ} \mathrm{C}$ 5 minutes.

\section{Confocal imaging of transgenic larval brains}

Dissection and imaging were performed following the protocols in (Lee et al., 2018). In brief, fluorescence-positive $3^{\text {rd }}$ instar larvae were collected in 1x PBS solution and then cut in half and inverted to expose the brain. Brains were transferred into $1.5 \mathrm{~mL}$ centrifuge tubes and fixed in 4\% PFA in 1xPBS buffer for 20 minutes. Brains were then washed for 10 minutes three times in $0.2 \%$ PBST. Finally, samples were mounted on glass slides with $8 \mu \mathrm{L}$ of VectaShield (VectorLabs \#H-1000) and imaged at 20x zoom with a Nikon W1 dual laser spinning-disc confocal microscope. 


\section{Analysis of single cell sequencing data}

To identify genes with expression profiles that overlap with expression of genes replaced with KozakGAL4 sequences, we queried the data from third instar larval CNS scRNAseq data described in Ravenscroft et al. (2020). The data (http://scope.aertslab.org/\#/Larval Brain/*/welcome) were imported into Seurat (version 4.0.1). Cells expressing the selected genes, for which the KozakGAL4 allele was generated (e.g CG3770, CG10939, CG10947 and CG15093), were identified using WhichCells function and genes enriched in these cells were identified using FindMarkers with default parameters. A list of the top 10 genes that were minimally expressed outside the expression domain of genes with KozakGAL4 alleles was generated. We then selected genes from the list for which T2AGAL4 were generated and compared the expression profiles using available images.

\section{Western blots}

Flies were homogenized using Cell Lysis Buffer $(25 \mathrm{mM}$ Tris- $\mathrm{HCl}$ pH 7.5, $100 \mathrm{mM}$ $\mathrm{NaCl}, 1 \mathrm{mM}$ EDTA, 1\% Triton-X 100, $1 \mathrm{X}$ liquid protease inhibitor (Gen DEPOT), $0.1 \mathrm{M}$ DTT). The supernatant was collected after centrifugation at 13, $000 \mathrm{rpm}$ for $10 \mathrm{~min}$ at $4^{\circ} \mathrm{C}$ (Eppendorf 5424R with rotor Eppendorf FA-45-24-11). The supernatant was mixed with Laemmli Buffer containing $\beta$-mercaptoethanol and heated at $95^{\circ} \mathrm{C}$ for $10 \mathrm{~min}$. Subsequently, the samples were loaded in 4-20\% gradient polyacrylamide gels (Bio-Rad Mini-PROTEAN ${ }^{\circledR}$ TGX $^{\mathrm{TM}}$ ). Following electrophoresis, proteins were transferred onto a polyvinylidene difluoride membrane (Immobilon, Sigma). The membrane was blocked using skimmed milk and treated with the primary antibody for overnight. The following antibodies were used in the present study: rabbit anti-GFP (1:1000) (Thermo Fisher Scientific, \#A-11122), mouse anti-Actin (1:5000) (EMD Millipore, \#MAB1501). Horseradish peroxidase-conjugated secondary antibody was used to detect the respective primary antibody. Blots were imaged on a Bio-Rad ChemiDocMP.

\section{Cloning of Wdr37-KIGFP constructs}

The fragments that position the GFP tag to the selected sites were PCR amplified from genomic DNA, sfGFP was amplified from pBS_SA_sfGFP_SD (Kanca et al., 2019a) and scarless DSred from pScarlessHD-DsRed (pScarlessHD-DsRed was a gift from Kate O'Connor-Giles (Addgene plasmid \# 64703 ; 
http://n2t.net/addgene:64703 ; RRID:Addgene_64703). The fragments were used together with homology donor intermediate for Wdr37 gene used for generating KozakGAL4 allele (CR70111) cut with Bbsl-HF to assemble NEB-HiFi DNA assembly following manufacturer's instructions. Schematics of HiFi assembly can be found in Figure 5 Supplementary figures 1, 2 and 3.

\section{Figure Legends}

Figure 1. KozakGAL4 strategy can be used to generate GAL4 gene trap alleles for approximately $\mathbf{5 0 \%}$ of Drosophila genes. (A) Analysis of Drosophila genome indicates that about half of the Drosophila genes do not have a suitably large coding intron for insertion of a T2AGAL4 cassette. These genes are on average shorter and have fewer genetic reagents compared to the genes that have a suitably large coding intron for inserting T2AGAL4 cassette. (B) Schematics of the KozakGAL4 targeting. Gray boxes, UTRs; orange box, gene coding region.

Figure 2. KozakGAL4 alleles document intricate gene expression patterns in third instar larval brains. (A)Examples of third instar larval brain gene expression patterns obtained by crossing KozakGAL4 allele of indicated genes with UASCD8mCherry flies. (B) The imaging results of reporter expression generated with KozakGAL4 allele were compared to the expression pattern of genes that are expressed in similar cells by analysis of single cell sequencing data imaged using T2AGAL4 alleles. Images are taken by crossing the GAL4 alleles with UASCD8mCherry. Arrowheads point to the shared expression pattern.

Figure 3. int200 strategy results in similar transgenesis success rates as the long homology arms CRIMICs. (A) Schematics of the int200 strategy. (B) Transgenesis data using int200_T2AGAL4 or int200_KozakGAL4 strategies.

Figure 4. gRNA_int200 strategy increases the transgenesis success rates. (A) Schematics of the gRNA_int200 strategy. (B) Transgenesis data using gRNA_int200_T2AGAL4 or 2XgRNA_int200_KozakGAL4 strategies.

Figure 5. 2XgRNA_int200 strategy can be used to tag any gene at any coding region to generate protein trap alleles. $(A)$ Schematics of the targeting constructs 
to integrate sfGFP protein tag at an N-terminal, internal or C-terminal location in Wdr37 gene locus. (B) Western blot analysis from adult flies show full length protein in all protein trap alleles with the arrow indicating the $81 \mathrm{kDa}$ band that is the length predicted for the Wdr37 protein fused to sfGFP.

Supplementary Figure 1. Alternative strategies to generate gene trap alleles in genes without suitable introns. For genes that cannot be targeted by artificial exon strategies and where suitable sgRNAs could not be found in the 5'UTR an artificial exon with SA_KozakGAL4 can be inserted in an intron in the 5'UTR (A) or in a short intron by deleting the exons following the intron $(B)$.

\section{Supplementary Figure 2. Identification of genes expressed in similar cells to} the KozakGAL4 alleles expressed in restricted patterns. (A) The single cell sequencing data from Ravenscroft et al. 2020 with the cells expressing the gene targeted by KozakGAL4 marked by red circles and cells expressing the gene targeted with T2AGAL4 allele marked by green circles. The imaging results of reporter expression generated with KozakGAL4 alleles were compared to the expression patterns of genes that are expressed in similar cells by analysis of single cell sequencing data imaged using T2AGAL4 alleles. Images are taken by crossing the GAL4 alleles with UAS-CD8mCherry. Arrowheads show the regions with the most overlap. (B) Cluster of trachea markers in the scRNA data from Ravenscroft et al. 2020.

\section{Supplementary Table 1. Analysis of Drosophila genome for the presence of suitable introns.}

\section{Supplementary Table 2. List of the $\mathbf{4 2 8}$ alleles generated in this study}

The alleles are indicated in tabs corresponding to the strategy used to generate the allele.

\section{References}


Bateman, J. R., Lee, A. M. and Wu, C. T. (2006) 'Site-specific transformation of Drosophila via phiC31 integrase-mediated cassette exchange', Genetics, 173(2): pp. 769-77.

Bellen, H. J., O'Kane, C. J., Wilson, C., Grossniklaus, U., Pearson, R. K. and Gehring, W. J. (1989) 'P-element-mediated enhancer detection: a versatile method to study development in Drosophila', Genes Dev, 3(9): pp. 1288-300.

Bier, E., Harrison, M. M., O'Connor-Giles, K. M. and Wildonger, J. (2018) 'Advances in Engineering the Fly Genome with the CRISPR-Cas System', Genetics, 208(1): pp. $1-18$.

Brand, A. H., and Perrimon N. (1993) 'Targeted gene expression as a means of altering cell fates and generating dominant phenotypes', Development, 118: pp. 40115.

Cavener, D. R. (1987) 'Comparison of the consensus sequence flanking translational start sites in Drosophila and vertebrates', Nucleic Acids Res, 15(4): pp. 1353-61.

Chen, C. H., Lin, H. Y., Pan, C. L. and Chen, F. C. (2011) 'The plausible reason why the length of $5^{\prime}$ untranslated region is unrelated to organismal complexity', BMC Res Notes, 4: pp. 312.

Diao, F., Ironfield, H., Luan, H., Shropshire, W. C., Ewer, J., Marr, E., Potter, C. J., Landgraf, M. and White, B. H. (2015) 'Plug-and-play genetic access to drosophila cell types using exchangeable exon cassettes', Cell Rep, 10(8): pp. 1410-21.

Garcia-Marques, J., Yang, C. P., Espinosa-Medina, I., Mok, K., Koyama, M. and Lee, T. (2019) 'Unlimited Genetic Switches for Cell-Type-Specific Manipulation', Neuron, 104(2): pp. 227-238.e7.

Gloor, G. B., Preston, C. R., Johnson-Schlitz, D. M., Nassif, N. A., Phillis, R. W., Benz, W. K., Robertson, H. M. and Engels, W. R. (1993) 'Type I repressors of P element mobility', Genetics, 135(1): pp. 81-95.

Gnerer, J. P., Venken, K. J. and Dierick, H. A. (2015) 'Gene-specific cell labeling using MiMIC transposons', Nucleic Acids Res, 43(8): pp. e56.

Gohl, D. M., Silies, M. A., Gao, X. J., Bhalerao, S., Luongo, F. J., Lin. C-C., Potter, C. J.. Clandinin, T. R. (2011) 'A versatile in vivo system for directed dissection of gene expression patterns', Nat Methods, 8: 231-7.

Gratz, S. J., Ukken, F. P., Rubinstein, C. D., Thiede, G., Donohue, L. K., Cummings, A. M., O'Connor-Giles, K. M. (2014) 'Highly specific and efficient CRISPR/Cas9catalyzed homology-directed repair in Drosophila', Genetics, 196(4): pp. 961-71.

Hacker, U., Nystedt S., Barmchi, M. P., Horn, C., Wimmer, E. A. (2003) 'piggyBacbased insertional mutagenesis in the presence of stably integrated $P$ elements in Drosophila', Proc. Natl. Acad. Sci., 100(13): pp. 7720-5. 
Jan, C. H., Friedman, R. C., Ruby, J. G. and Bartel, D. P. (2011) 'Formation, regulation and evolution of Caenorhabditis elegans 3'UTRs', Nature, 469(7328): pp. 97-101.

Jenett, A., Rubin, G. M., Ngo, T. T., Shepherd, D., Murphy, C., Dionne, H., Pfeiffer, B. D., Cavallaro, A., Hall, D., Jeter, J., lyer, N., Fetter, D., Hausenfluck, J. H., Peng, H., Trautman, E. T., Svirskas, R. R., Myers, E. W., Iwinski, Z. R., Aso, Y., DePasquale, G. M., Enos, A., Hulamm, P., Lam, S. C., Li, H. H., Laverty, T. R., Long, F., Qu, L., Murphy, S. D., Rokicki, K., Safford, T., Shaw, K., Simpson, J. H., Sowell, A., Tae, S., Yu, Y. and Zugates, C. T. (2012) 'A GAL4-driver line resource for Drosophila neurobiology', Cell Rep, 2(4): pp. 991-1001.

Kanca, O., Zirin, J., Garcia-Marques, J., Knight, S. M., Yang-Zhou, D., Amador, G., Chung, H., Zuo, Z., Ma, L., He, Y., Lin, W. W., Fang, Y., Ge, M., Yamamoto, S., Schulze, K. L., Hu, Y., Spradling, A. C., Mohr, S. E., Perrimon, N. and Bellen, H. J. (2019a) 'An efficient CRISPR-based strategy to insert small and large fragments of DNA using short homology arms', Elife, 8: e51539.

Kanca, O., Andrews, J.C., Lee, P.T., Patel, C., Braddock, S.R., Slavotinek, A.M., Cohen, J.S., Gubbels, C.S., Aldinger, K.A., Williams, J., Indaram, M., Fatemi, A., Yu, T.W., Agrawal, P.B., Vezina, G., Simons, C., Crawford, J., Lau, C.C.; Undiagnosed Diseases Network, Chung, W.K., Markello, T.C., Dobyns, W.B., Adams, D.R., Gahl, W.A., Wangler, M.F., Yamamoto, S., Bellen, H.J., Malicdan, M.C.V. (2019b) 'De novo variants in WDR37 are associated with epilepsy, colobomas, dysmorphism, developmental delay, intellectual disability, and cerebellar hypoplasia', Am J of Hum Genet, 105: pp. 413-24.

Kondo, S. and Ueda, R. (2013) 'Highly improved gene targeting by germline-specific Cas9 expression in Drosophila', Genetics, 195(3): pp. 715-21.

Kozak, M. (1986) 'Point mutations define a sequence flanking the AUG initiator codon that modulates translation by eukaryotic ribosomes', Cell, 44(2): pp. 283-92.

Larkin, A., Marygold, S.J., Antonazzo, G., Attrill, H., dos Santos, G., Garapati, P.V., Goodman, J.L., Gramates, L.S., Millburn, G., Strelets, V.B., Tabone, C.J., Thurmond, J. and the FlyBase Consortium (2021) 'FlyBase: updates to the Drosophila melanogaster knowledge base', Nucleic Acids Res. 49(D1): D899-D907.

Lee, P. T., Zirin, J., Kanca, O., Lin, W. W., Schulze, K. L., Li-Kroeger, D., Tao, R., Devereaux, C., Hu, Y., Chung, V., Fang, Y., He, Y., Pan, H., Ge, M., Zuo, Z., Housden, B. E., Mohr, S. E., Yamamoto, S., Levis, R. W., Spradling, A. C., Perrimon, N. and Bellen, H. J. (2018) 'A gene-specific T2A-GAL4 library for Drosophila', Elife, 7: e35574.

Li-Kroeger D., Kanca O., Lee P-T, Cowan S., Lee M. T., Jaiswal M., Salazar, J. L., He Y., Zuo Z., Bellen H. J. (2018) 'An expanded toolkit for gene tagging based on MiMIC and scarless CRISPR tagging in Drosophila', Elife, 7: e38709. 
Lukacsovich, T., Asztalos, Z., Awano, W., Baba, K., Kondo, S., Niwa, S., Yamamoto, D. (2001) 'Dual-tagging gene trap of novel genes in Drosophila melanogaster', Genetics, 157: pp. 727-42.

Luschnig, S., Bätz, T., Armbruster, K. and Krasnow, M. A. (2006) 'serpentine and vermiform encode matrix proteins with chitin binding and deacetylation domains that limit tracheal tube length in Drosophila', Curr Biol, 16(2): pp. 186-94.

Manning, L., Heckscher, E. S., Purice, M. D., Roberts, J., Bennett, A. L., Kroll, J. R., Pollard, J. L., Strader, M. E., Lupton, J. R., Dyukareva, A. V., Doan, P. N., Bauer, D. M., Wilbur, A. N., Tanner, S., Kelly, J. J., Lai, S. L., Tran, K. D., Kohwi, M., Laverty, T. R., Pearson, J. C., Crews, S. T., Rubin, G. M. and Doe, C. Q. (2012) 'A resource for manipulating gene expression and analyzing cis-regulatory modules in the Drosophila CNS', Cell Rep, 2(4): pp. 1002-13.

Mayer, L. R., Diegelmann, S., Abassi, Y., Eichinger, F. and Pflugfelder, G. O. (2013) 'Enhancer trap infidelity in Drosophila optomotor-blind', Fly (Austin), 7(2): pp. 118-28.

Nagarkar-Jaiswal S., Lee P-T, Campbell M. E., Chen K., Anguiano-Zarate S.,Gutierrez M. C., Busby T., Lin W. W., He Y., Schulze K. L., Booth B. W., EvansHolm M., Venken K. J., Levis R. W., Spradling A. C., Hoskins R. A., Bellen H. J. (2015a) 'A library of MiMICs allows tagging of genes and reversible, spatial and temporal knockdown of proteins in Drosophila', Elife, 4: e05338.

Nagarkar-Jaiswal S., DeLuca S. Z., Lee P-T, Lin W. W., Pan H., Zuo Z., Lv J., Spradling A. C., Bellen H.J. (2015b) 'A genetic toolkit for tagging intronic MiMIC containing genes', Elife, 4: e08469.

Nagarkar-Jaiswal S., Manivannan S. N., Zuo Z., Bellen H. J. (2017) 'A cell cycleindependent, conditional gene inactivation strategy for differentially tagging wild-type and mutant cells', Elife, 31: e26420.

O'Kane, C. J. and Gehring, W. J. (1987) 'Detection in situ of genomic regulatory elements in Drosophila', Proc Natl Acad Sci U S A, 84(24): pp. 9123-7.

Pfeiffer, B. D., Jenett, A., Hammonds, A. S., Ngo, T. T., Misra, S., Murphy, C., Scully, A., Carlson, J. W., Wan, K. H., Laverty, T. R., Mungall, C., Svirskas, R., Kadonaga, J. T., Doe, C. Q., Eisen, M. B., Celniker, S. E. and Rubin, G. M. (2008) 'Tools for neuroanatomy and neurogenetics in Drosophila', Proc Natl Acad Sci U S A, 105(28): pp. 9715-20.

Port, F. and Bullock, S. L. (2016) 'Augmenting CRISPR applications in Drosophila with tRNA-flanked sgRNAs', Nat Methods, 13(10): pp. 852-4.

Port, F., Chen, H. M., Lee, T. and Bullock, S. L. (2014) 'Optimized CRISPR/Cas tools for efficient germline and somatic genome engineering in Drosophila', Proc Natl Acad Sci U S A, 111(29): pp. E2967-76.

Ravenscroft, T.A., Janssens, J., Lee, P.T., Tepe, B., Marcogliese, P.C., Makhzami, S., Holmes, T., Aerts, S., Bellen, H.J. (2020) 'Drosophila voltage-gated sodium 
channels are only expressed in active neurons and are localized to distal axonal initial segment-like domains', J Neurosci, 40: pp. 7999-8024.

Spradling, A. C., Stern, D. M., Kiss, I., Roote, J., Laverty, T. and Rubin, G. M. (1995) 'Gene disruptions using $P$ transposable elements: an integral component of the Drosophila genome project', Proc Natl Acad Sci U S A, 92(24): pp. 10824-30.

Spradling, A. C., Stern, D., Beaton, A., Rhem, E. J., Laverty, T., Mozden, N., Misra, S. and Rubin, G. M. (1999) 'The Berkeley Drosophila Genome Project gene disruption project: Single P-element insertions mutating $25 \%$ of vital Drosophila genes', Genetics, 153(1): pp. 135-77.

Venken, K. J., Schulze, K. L., Haelterman, N. A., Pan, H., He, Y., Evans-Holm, M., Carlson, J. W., Levis, R. W., Spradling, A. C., Hoskins, R. A. and Bellen, H. J. (2011) 'MiMIC: a highly versatile transposon insertion resource for engineering Drosophila melanogaster genes', Nat Methods, 8(9): pp. 737-43.

Wangler, M. F., Yamamoto, S., Chao, H. T., Posey, J. E., Westerfield, M., Postlethwait, J., Hieter, P., Boycott, K. M., Campeau, P. M., Bellen, H. J. and (UDN), M. o. t. U. D. N. (2017) 'Model Organisms Facilitate Rare Disease Diagnosis and Therapeutic Research', Genetics, 207(1): pp. 9-27.

Wilson, C., Pearson, R. K., Bellen, H. J., O'Kane, C. J., Grossniklaus, U. and Gehring, W. J. (1989) 'P-element-mediated enhancer detection: an efficient method for isolating and characterizing developmentally regulated genes in Drosophila', Genes Dev, 3(9): pp. 1301-13. 
Kanca et al. 2021 Figure 1. KozakGAL4 strategy

A B

CDS length

$<500$

$500-1000$

$1000-2000$

2000-3000

$3000-4000$

$4000-5000$

$>=5000$

avg

\# of genes

\section{with \\ without}

suitable intron suitable intron

412

1118

2262

965

479

241

310

2051

5787

759
1530
1887
554
151
54
62

1321

4997
Target locus

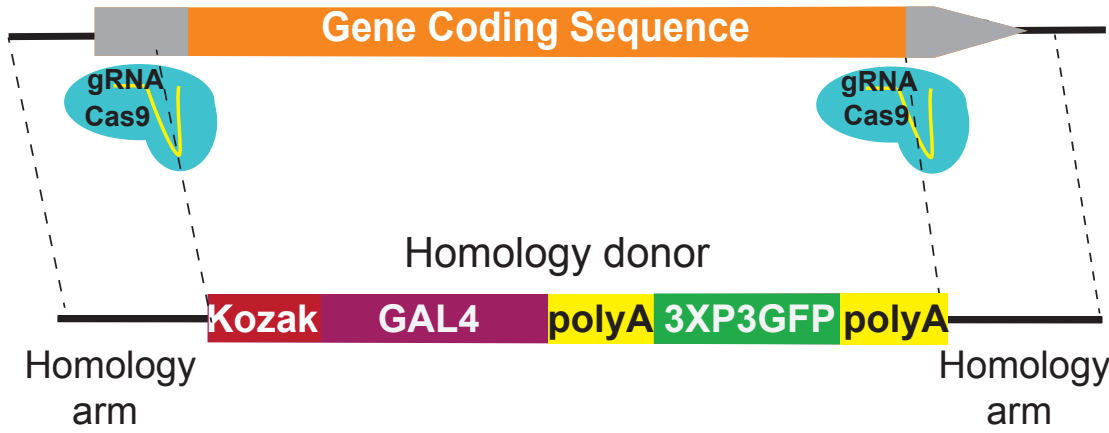

Resulting genomic locus 
Kanca et al. 2021 Figure 2. KozakGAL4 expression

A KozakGAL4 alleles document intricate gene expression patterns in 3rd instar larval brain

CG13737

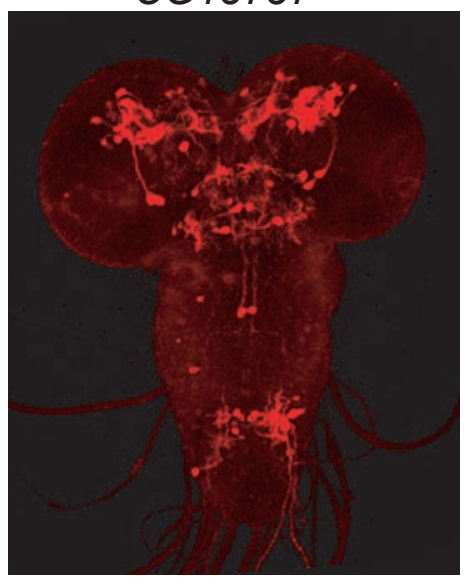

Sk1

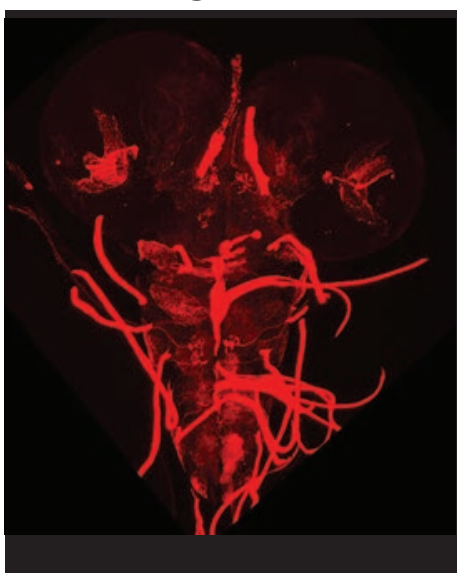

CG15744

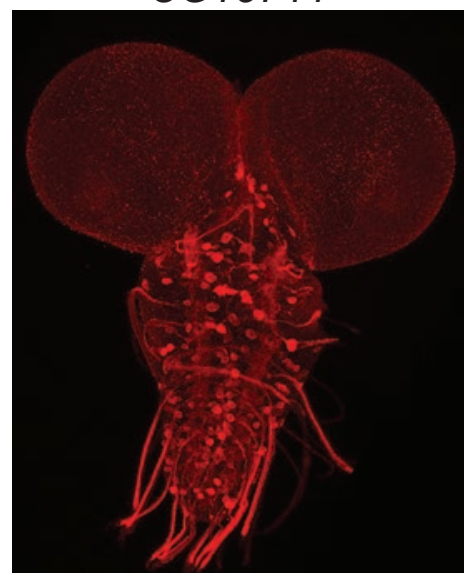

pex 2

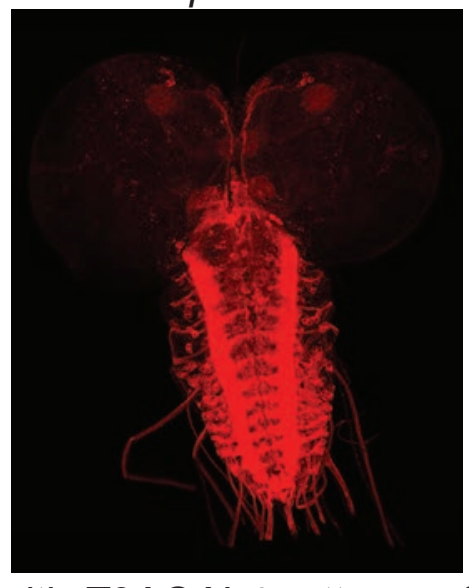

Usp7

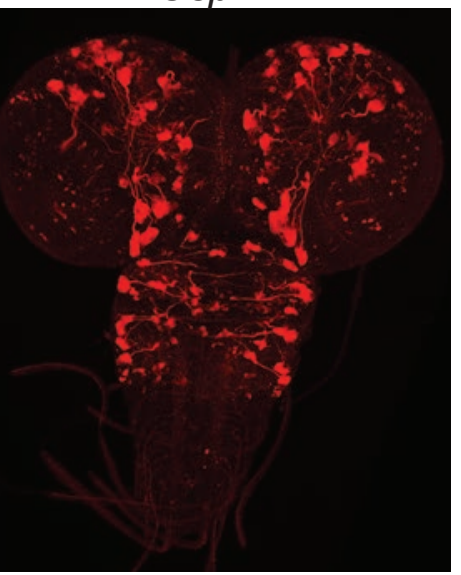

mtm

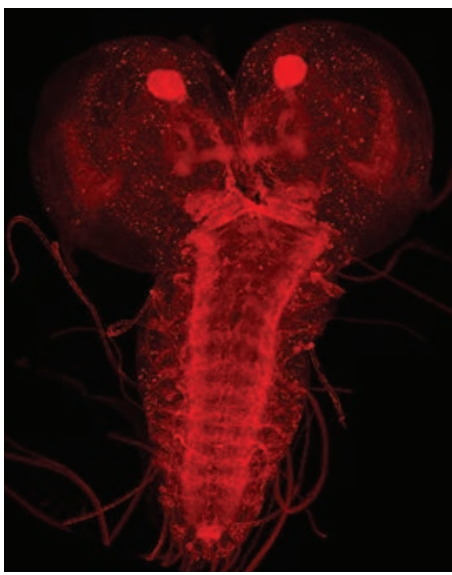

ufc 1

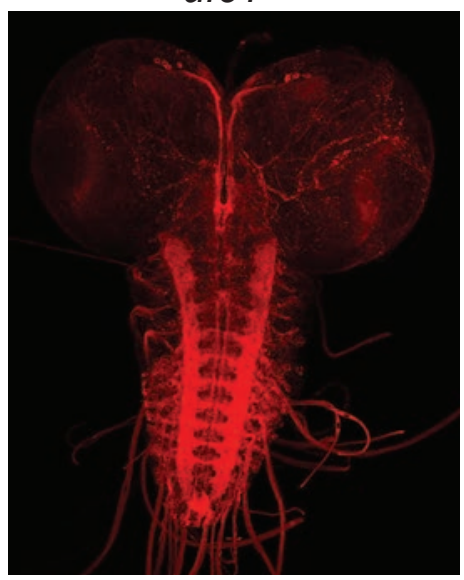

CG7504

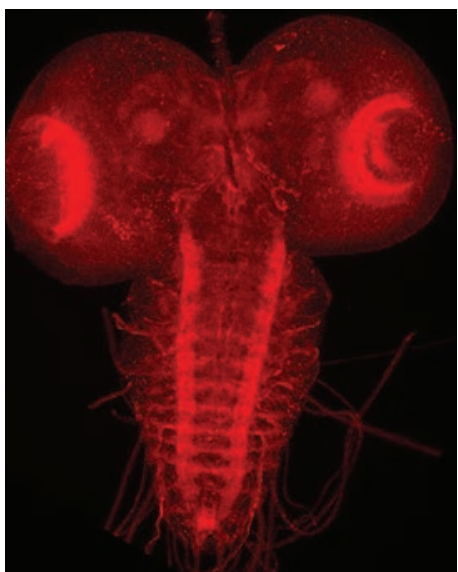

B KozakGAL4 patterns overlap with T2AGAL4 patterns of genes in the overlapping single cell sequencing clusters

CG10939-KozakGAL4

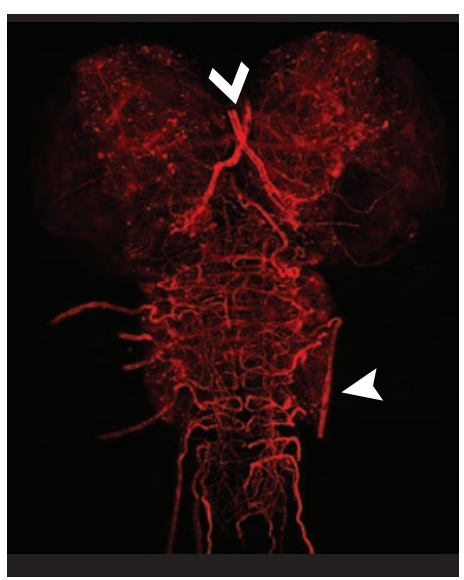

CG10939

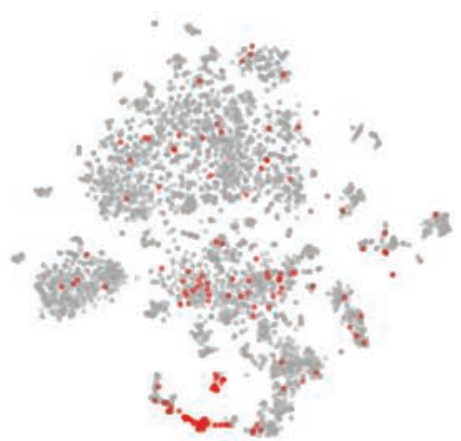

wbl-T2AGAL4

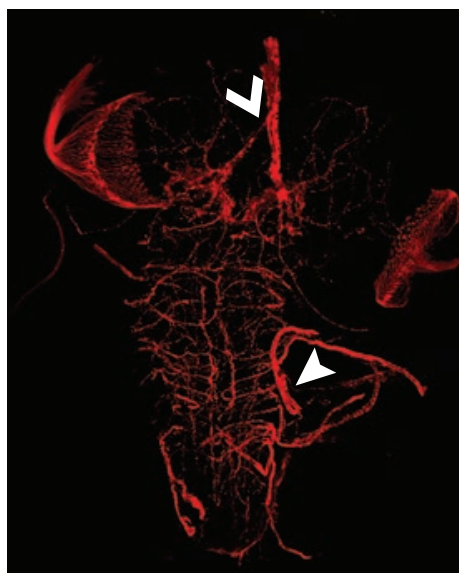

$w b l$

CG10939

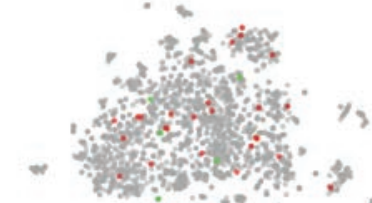

$-3$.
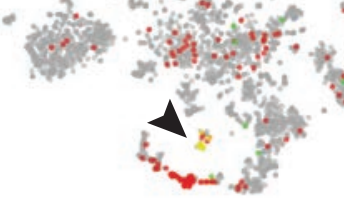

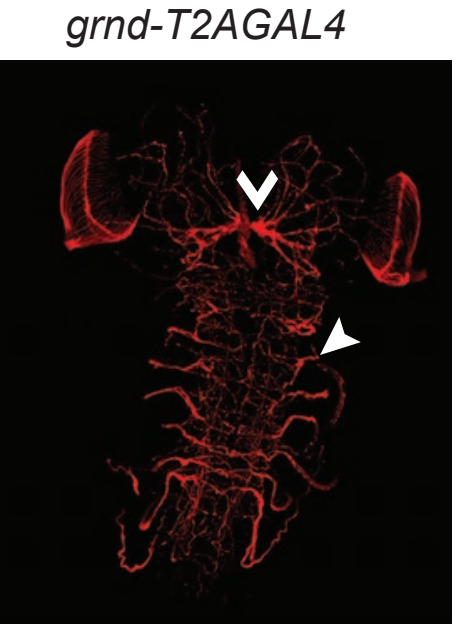

CG31121-T2AGAL4
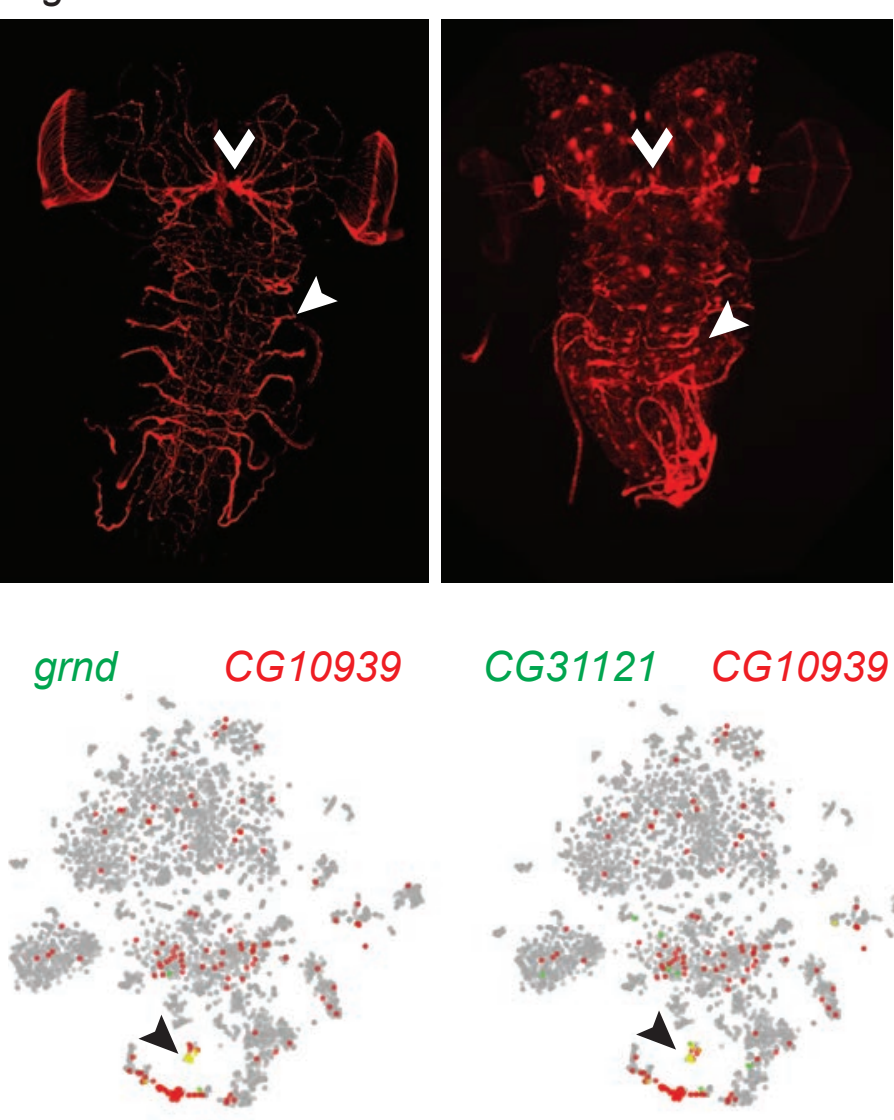
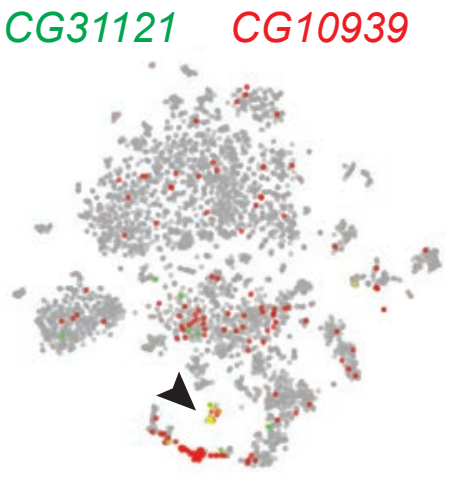
Kanca et al. 2021 Figure 3. int200 strategy

A Schematics of int200 strategy

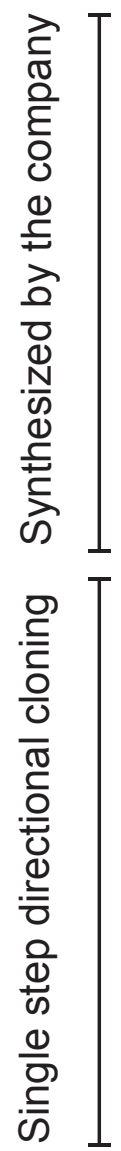

\section{U6:3-gRNA1}

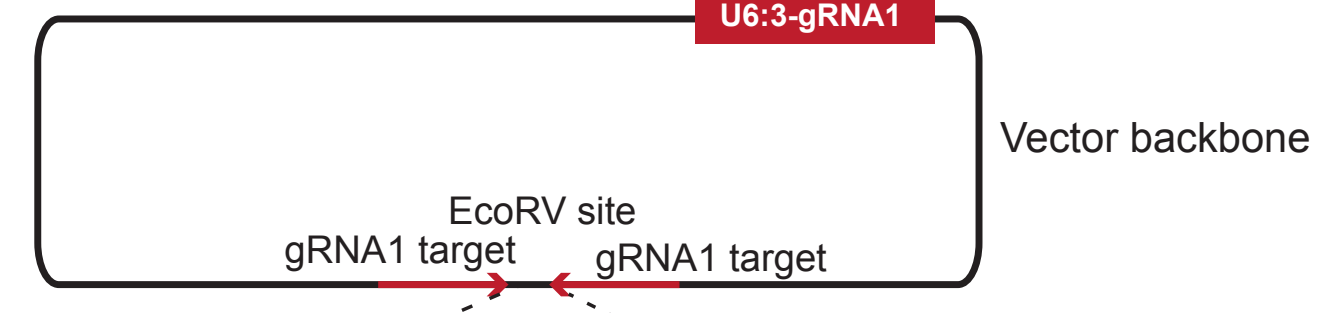

-2000bps Bbsl Bbsl 200bps -. Fragment to synthesize (460 bps)

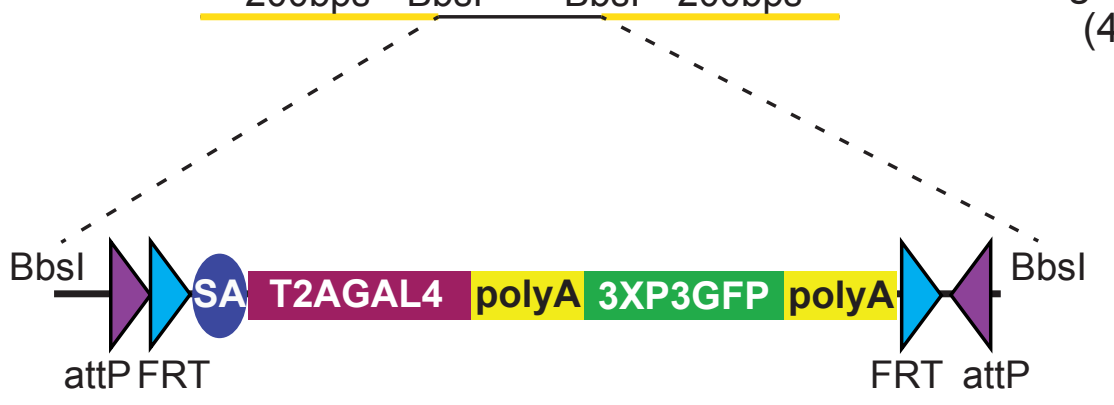

4847 bps

Bbsl

\section{Kozak GAL4}

polyA 3XP3GFP polyA

Bbsl

4273 bps

B Transgenesis rates of int200 strategy

\begin{tabular}{|l|r|r|r|}
\hline int200 strategy & Genes targeted & Successful integration & Transgenesis Rate \\
\hline T2AGAL4 & 397 & 252 & 63.48 \\
\hline KozakGAL4 & 36 & 22 & 61.11 \\
\hline
\end{tabular}


A Schematics of gRNA_int200 strategy
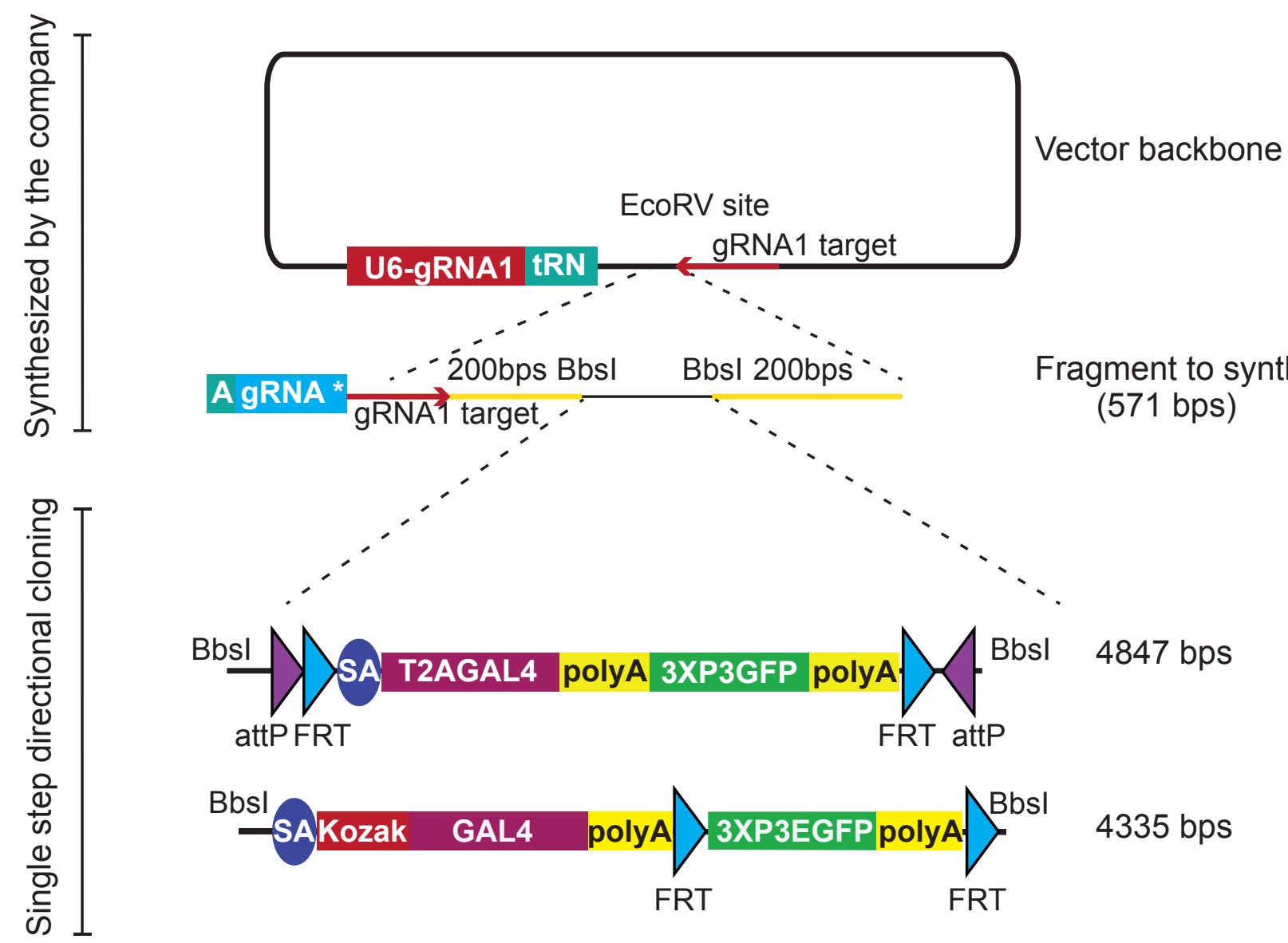

B Transgenesis rates of gRNA_int200 strategy

\begin{tabular}{|l|r|r|r|}
\hline gRNA_int200 strategy & Genes targeted & Successful integration & Transgenesis Rate \\
\hline T2AGAL4 & 127 & 95 & 74.80 \\
\hline KozakGAL4 & 72 & 59 & 81.94 \\
\hline
\end{tabular}




\section{A}

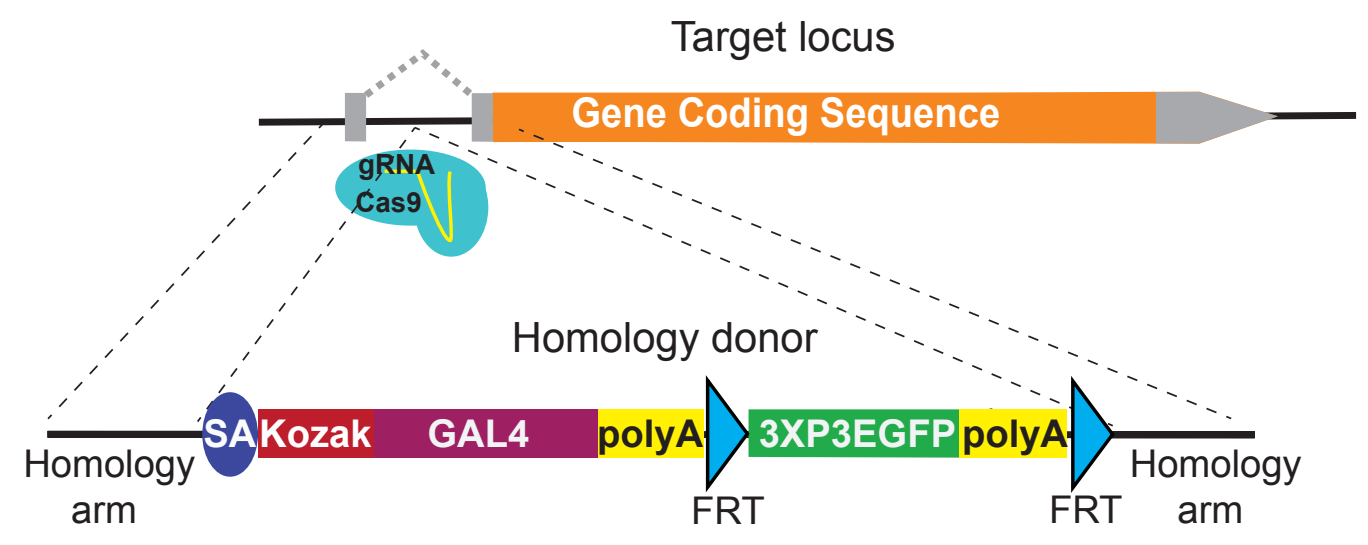

Resulting genomic locus

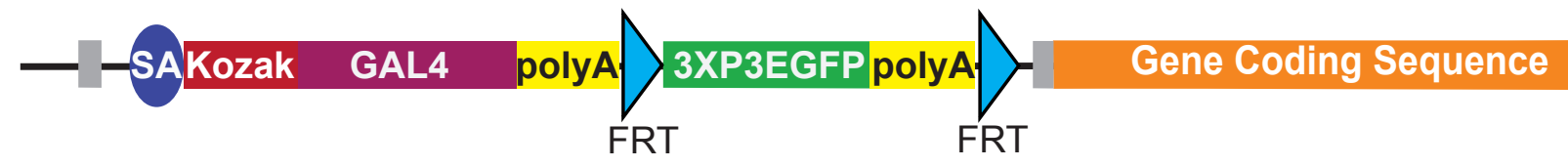

B

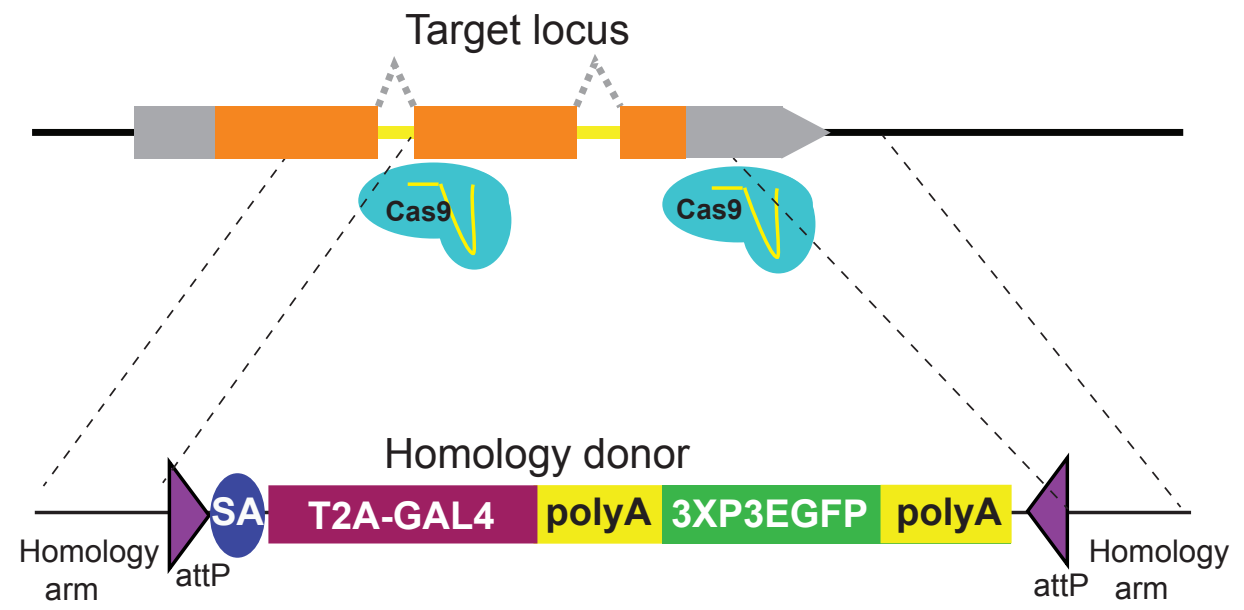

Resulting genomic locus

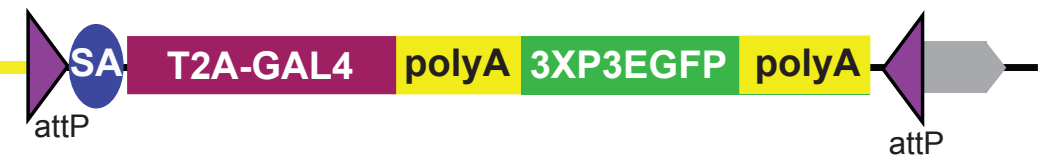


Kanca et al. 2021 Supplementary Figure 2. Single cell sequencing based overlaps in expression of indicated genes in third instar larval brain

A
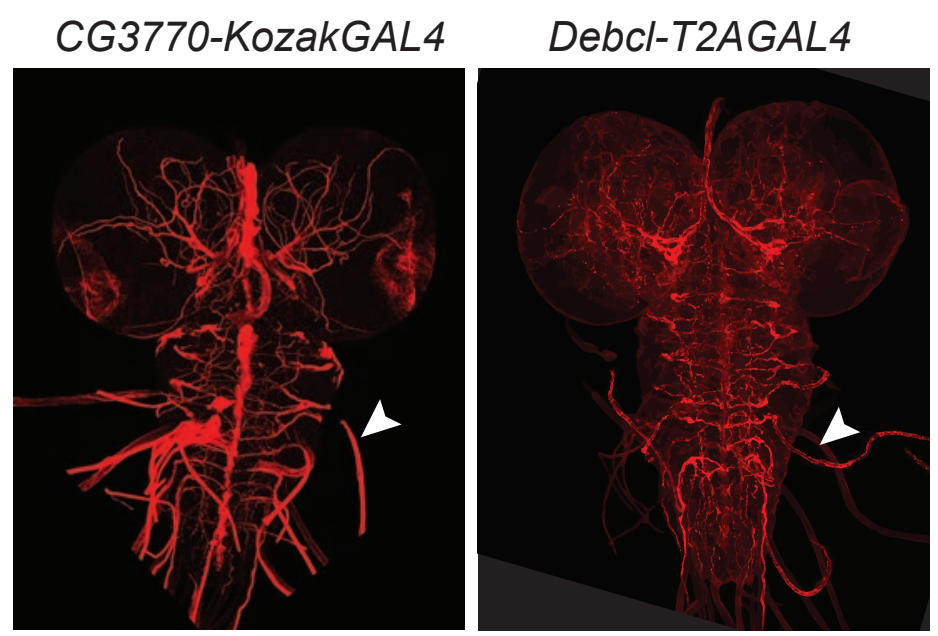

CG3770

Debcl
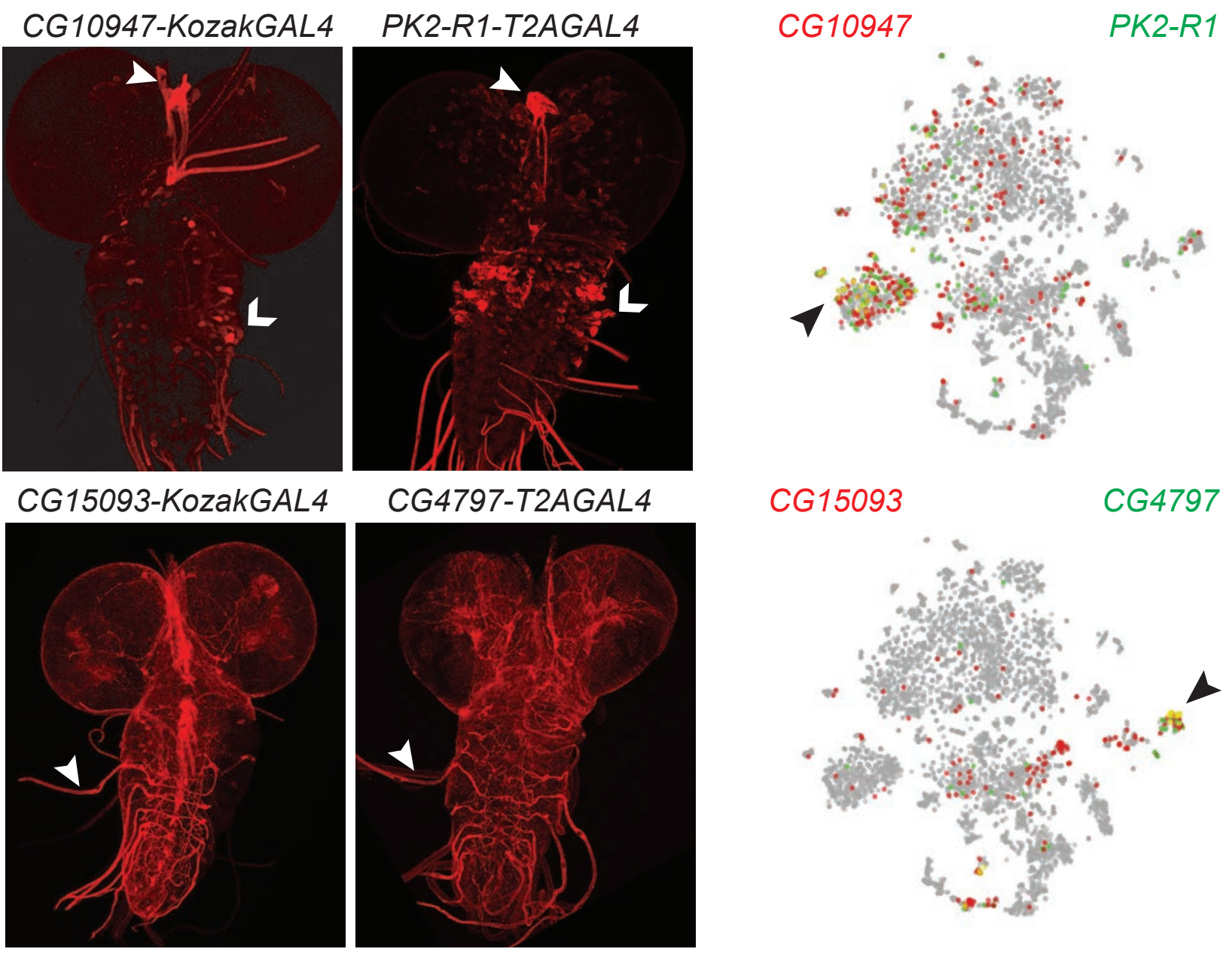

B
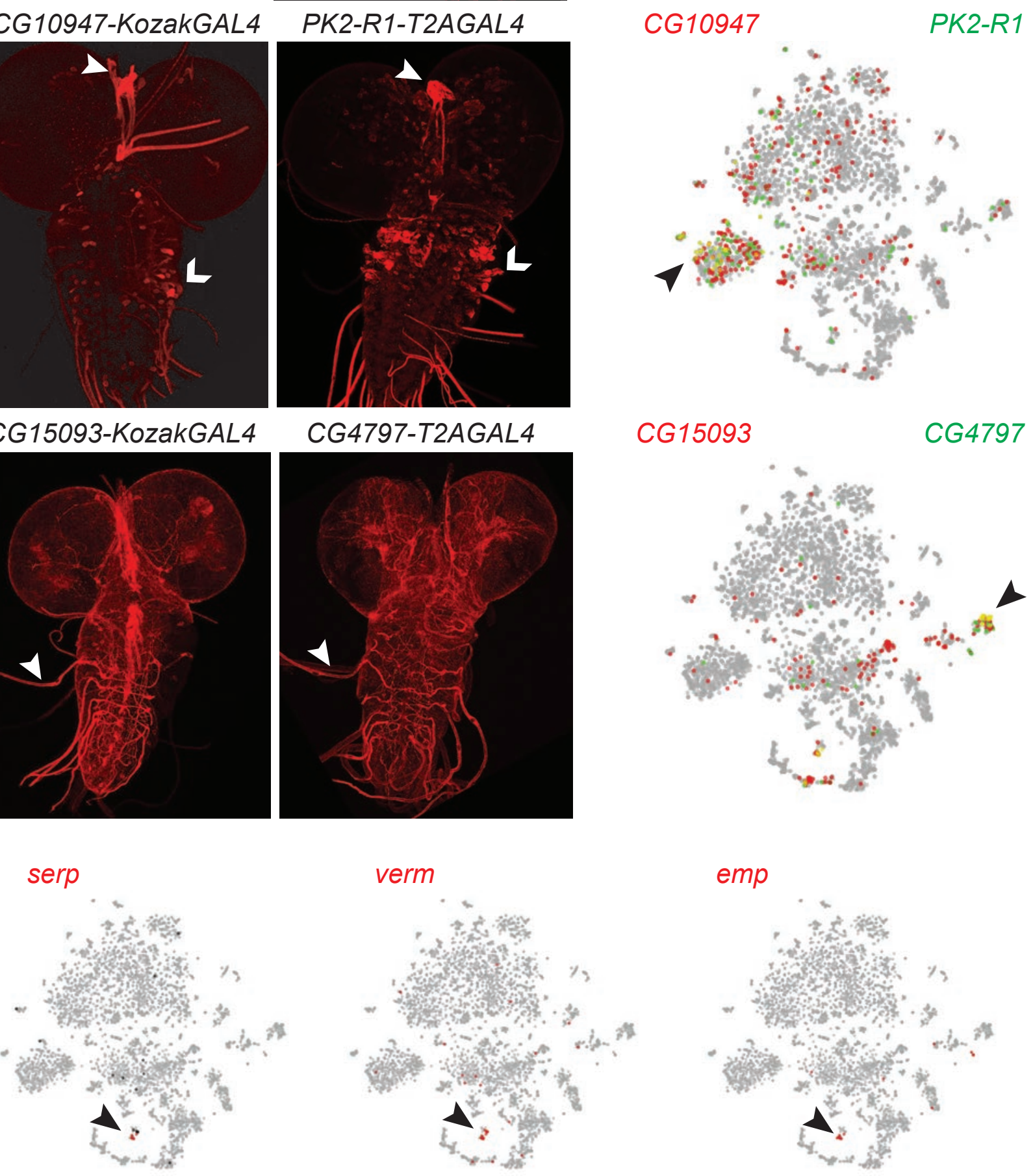


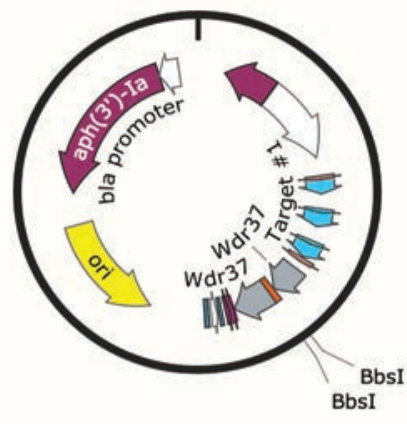
wdr37

GFP-NTerm

pUC57_Kan_gw_OK2_wdr37

Plasmid linearized by Bbsl cut 4022bps

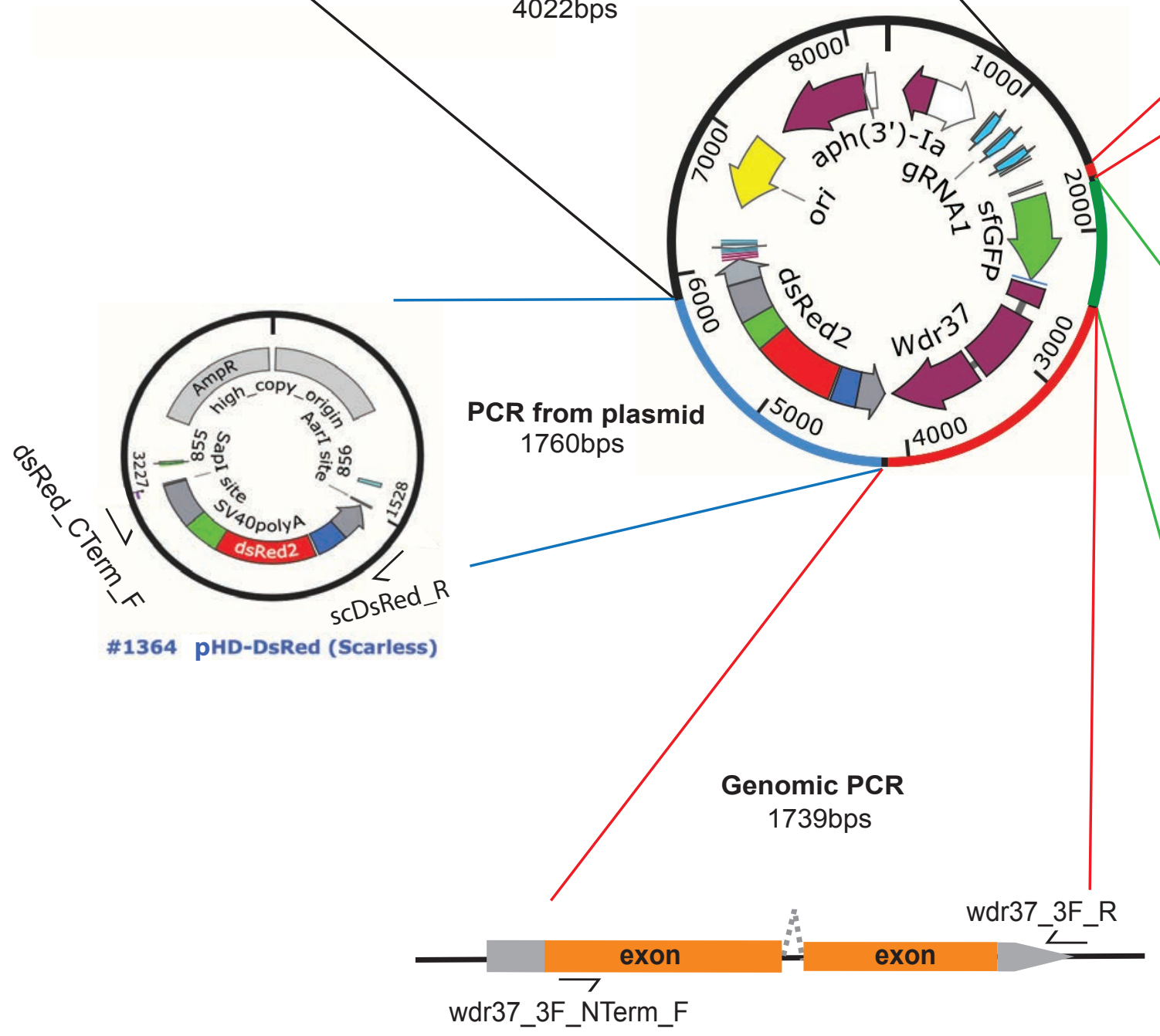

PCR from plasmid

799bps

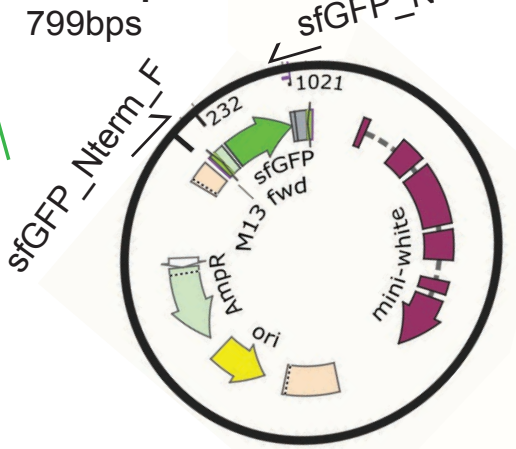

pScaffold_sfGFP_GT_pO 


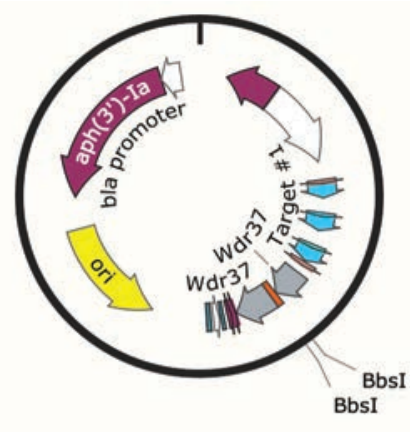

wdr37

GFP-int

pUC57_Kan_gw_OK2_wdr37

Plasmid linearized by Bbsl cut

4022bps
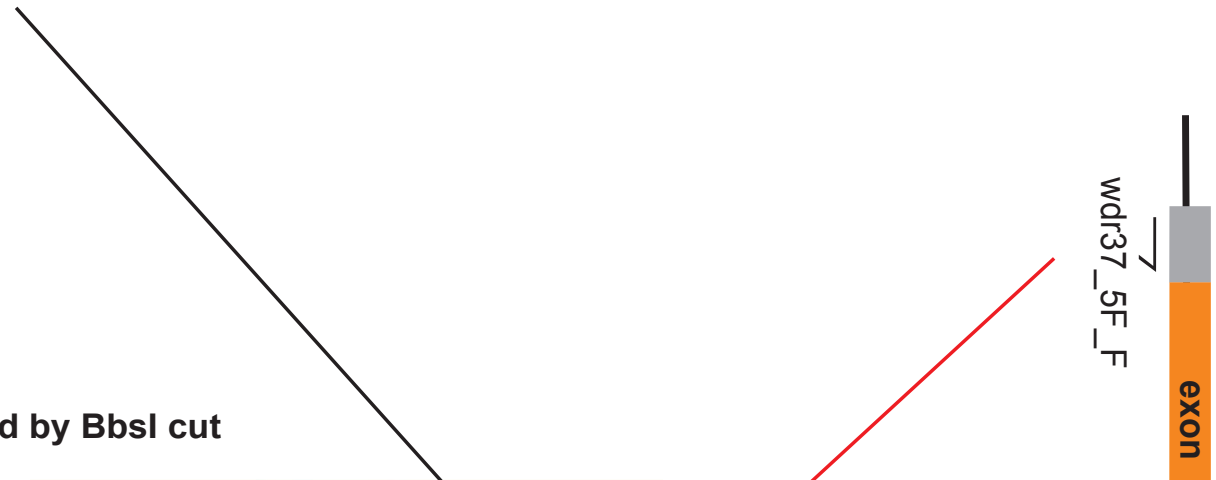

ำ

亭

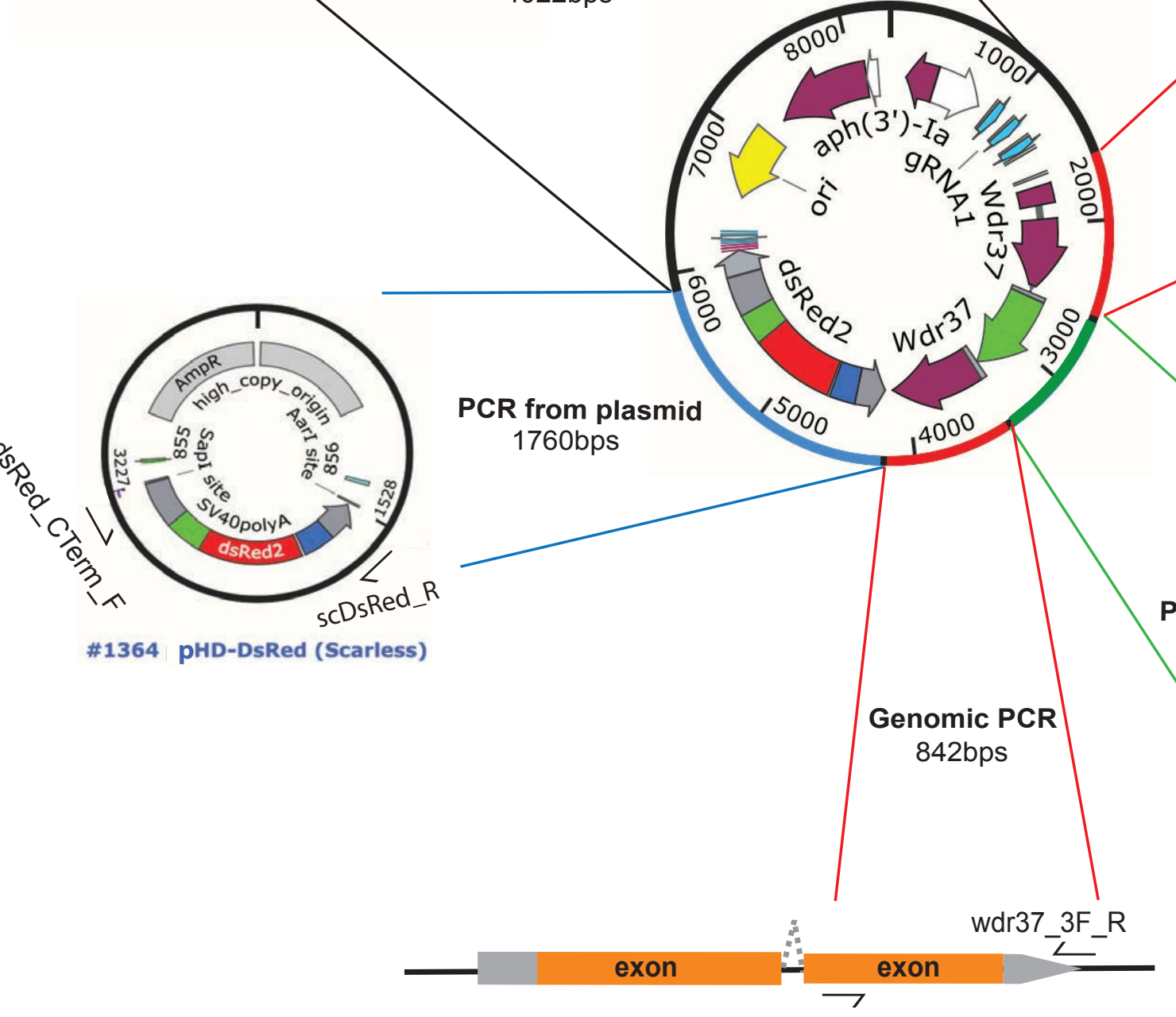

Genomic PCR 1049bps

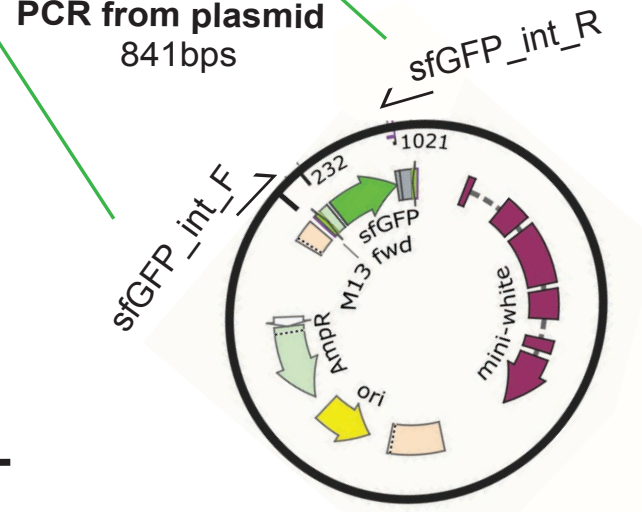

wdr37_3F_int_F 
bioRxiv preprint doi: https://doi.org/10.1101/2021.12.24.474112; this version posted December 24,2021 . The copyright holder for this

preprint (which was not certified by peer review) is the author/funder, who has granted bioRxiv a license to display the preprint in perpetuity. It is made available under aCC-BY 4.0 International license.

Supplementary Table 1. Analysis of Drosophila genome for the presence of suitable introns.

\begin{tabular}{l|r|r|}
\hline CDS length & crimicable & kozak target \\
\hline$<500$ & 412 & 759 \\
\hline $500-1000$ & 1118 & 1530 \\
\hline $1000-2000$ & 2262 & 1887 \\
\hline $2000-3000$ & 965 & 554 \\
\hline $3000-4000$ & 479 & 151 \\
\hline $4000-5000$ & 241 & 54 \\
\hline$>=5000$ & 310 & 62 \\
\hline & 2051 & 1321 \\
avg & 5787 & 4997 \\
all genes
\end{tabular}

\begin{tabular}{|l|r|r|}
\hline Annotated alleles & crimicable & kozak target \\
\hline 0 & 27 & 110 \\
\hline $1-10$ & 3069 & 3867 \\
\hline $11-20$ & 1380 & 648 \\
\hline $21-30$ & 476 & 185 \\
\hline $31-40$ & 265 & 67 \\
\hline $41-50$ & 156 & 42 \\
\hline$>50$ & 414 & 78 \\
\hline & & \\
avg & 18.5 & 9 \\
all genes & 5787 & 4997
\end{tabular}
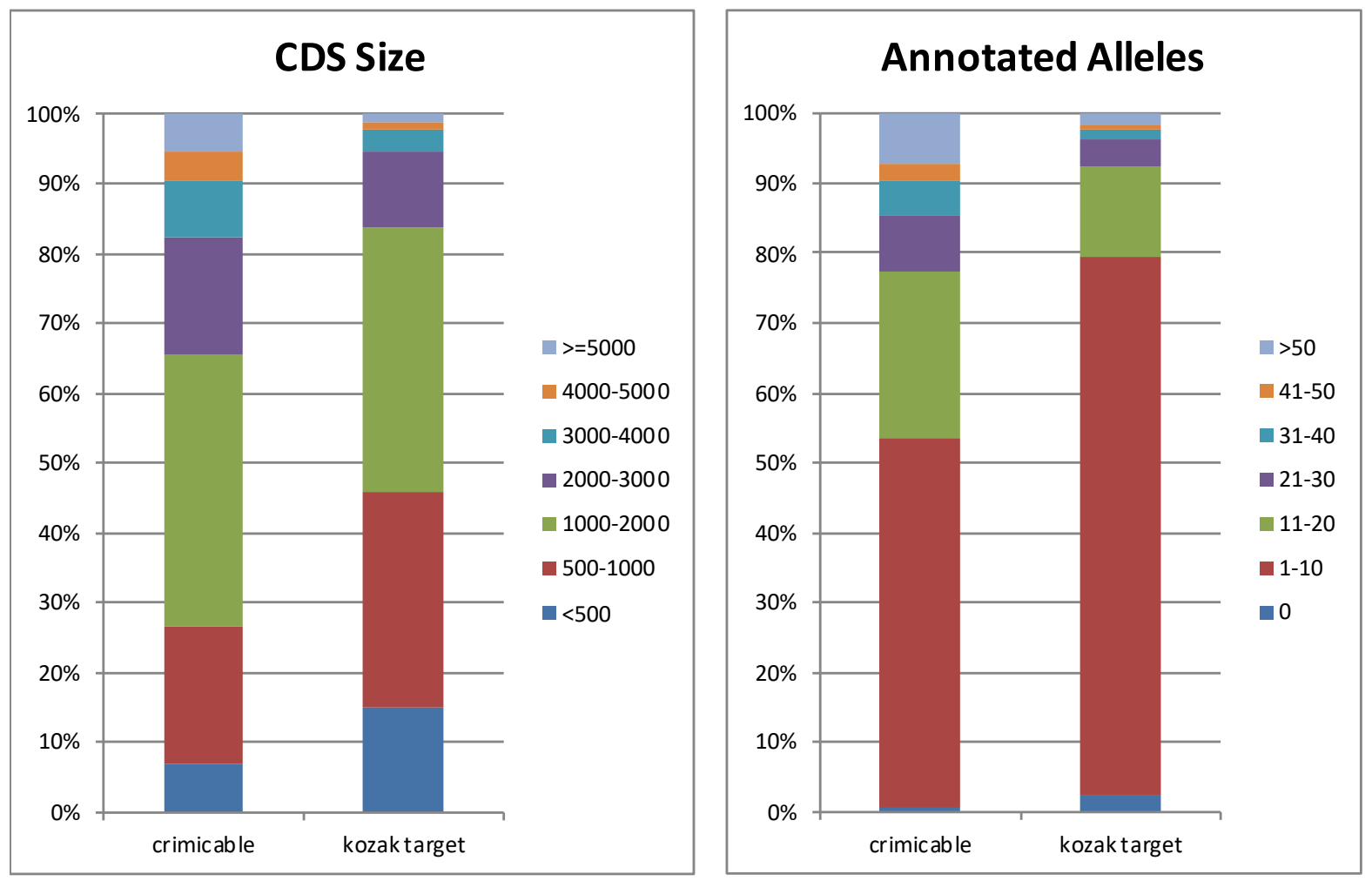
bioRxiv preprint doi: https://doi.org/10.1101/2021.12.24.474112; this version posted December $24,2021$. The copyright holder for this preprint (which was not certified by peer review) is the author/funder, who has granted bioRxiv a license to display the preprint in perpetuity. It is made available under aCC-BY 4.0 International license.

Supplementary Table 2. List of the 428 alleles generated in this study int200_T2AGAL4 strategy

\begin{tabular}{|c|c|c|c|c|}
\hline CR & Gene & Chr. & Donor DNA & FlyBase FBgn \\
\hline$\overline{\mathrm{CR} 02214}$ & $\overline{\mathrm{Ge}-1}$ & 2 & int200_pM37_p1 & FBgn0283682 \\
\hline CR02215 & $\operatorname{trp}$ & 3 & int200_pM37_p2 & FBgn0003861 \\
\hline CR02222 & REPTOR-BP & 2 & int200_pM37_p0 & FBgn0032202 \\
\hline CR02226 & hang & $\mathrm{X}$ & int200_pM37_p0 & FBgn0026575 \\
\hline CR02229 & Spt6 & $\bar{X}$ & int200_pM37_p1 & FBgn0028982 \\
\hline CR02234 & CG4115 & 3 & int200_pM37_p0 & FBgn0038017 \\
\hline CR02237 & $\mathrm{I}(2) \mathrm{k} 05911$ & 2 & int200_pM37_p1 & FBgn0284244 \\
\hline CR02239 & $\mathrm{mmd}$ & $\bar{X}$ & int200_pM37_p1 & FBgn0259110 \\
\hline CR02242 & CG1371 & 2 & int200_pM37_p1 & FBgn0033482 \\
\hline CR02244 & Cirl & 2 & int200_pM37_p2 & FBgn0033313 \\
\hline CR02253 & yellow-h & 4 & int200_pM37_p0 & FBgn0039896 \\
\hline CR02254 & Fatp3 & 2 & int200_pM37_p0 & FBgn0034999 \\
\hline CR02257 & ey & 4 & int200_pM37_p1 & FBgn0005558 \\
\hline CR02258 & nAChRalpha3 & $\bar{X}$ & int200_pM37_p1 & FBgn0015519 \\
\hline CR02259 & nAChRbeta2 & 3 & int200_pM37_p1 & FBgn0004118 \\
\hline CR02260 & side-II & 2 & int200_pM37_p1 & FBgn0259213 \\
\hline CR02261 & ATPsynbeta & 4 & int200_pM37_p1 & FBgn0010217 \\
\hline CR02262 & myo & 4 & int200_pM37_p1 & FBgn0026199 \\
\hline CR02267 & Tace & 3 & int200_pM37_p1 & FBgn0039734 \\
\hline CR02270 & poe & 2 & int200_pM37_p2 & FBgn0011230 \\
\hline CR02279 & lectin-46Ca & 2 & int200_pM37_p0 & FBgn0040093 \\
\hline CR02280 & $\mathrm{He}$ & 2 & int200_pM37_p0 & FBgn0028430 \\
\hline CR02283 & TbCMF46 & 2 & int200_pM37_p0 & FBgn0032163 \\
\hline CR02284 & CG30383 & 2 & int200_pM37_p0 & FBgn0050383 \\
\hline CR02285 & Adgf-E & 2 & int200_pM37_p0 & FBgn0033952 \\
\hline CR02287 & $\mathrm{Ns2}$ & 2 & int200_pM37_p0 & FBgn0034243 \\
\hline CR02289 & CG44434 & 2 & int200_pM37_p0 & FBgn0265626 \\
\hline CR02292 & Cyp9h1 & 2 & int200_pM37_p0 & FBgn0033775 \\
\hline CR02293 & Usp14 & 2 & int200_pM37_p0 & FBgn0032216 \\
\hline CR02296 & Gr39b & 2 & int200_pM37_p0 & FBgn0041245 \\
\hline CR02298 & Cul3 & 2 & int200_pM37_p0 & FBgn0261268 \\
\hline CR02303 & CG14339 & 2 & int200_pM37_p0 & FBgn0031301 \\
\hline CR02304 & Ugt36F1 & 2 & int200_pM37_p0 & FBgn0027074 \\
\hline CR02306 & CG2772 & 2 & int200_pM37_p0 & FBgn0031533 \\
\hline CR02308 & CG33509 & 2 & int200_pM37_p0 & FBgn0053509 \\
\hline CR02309 & CG31816 & 2 & int200_pM37_p0 & FBgn0051816 \\
\hline CR02310 & CG3907 & 2 & int200_pM37_p0 & FBgn0034958 \\
\hline CR02311 & Wdr59 & 2 & int200_pM37_p0 & FBgn0032339 \\
\hline CR02315 & rhi & 2 & int200_pM37_p0 & FBgn0004400 \\
\hline CR02316 & CG17974 & 2 & int200_pM37_p0 & FBgn0034624 \\
\hline CR02319 & CG34430 & 2 & int200_pM37_p0 & FBgn0085459 \\
\hline CR02327 & CG34205 & 2 & int200_pM37_p0 & FBgn0085234 \\
\hline CR02329 & CG17768 & 2 & int200_pM37_p0 & FBgn0032240 \\
\hline CR02336 & CG4294 & 2 & int200_pM37_p0 & FBgn0034742 \\
\hline CR02341 & lok & 2 & int200_pM37_p0 & FBgn0019686 \\
\hline
\end{tabular}


bioRxiv preprint doi: https://doi.org/10.1101/2021.12.24.474112; this version posted December $24,2021$. The copyright holder for this preprint (which was not certified by peer review) is the author/funder, who has granted bioRxiv a license to display the preprint in perpetuity. It is made available under aCC-BY 4.0 International license.

\begin{tabular}{|c|c|c|c|c|}
\hline CR02342 & CG6220 & 2 & int200_pM37_p0 & FBgn0033865 \\
\hline CR02343 & ACXE & 2 & int200_pM37_p0 & FBgn0040506 \\
\hline CR02344 & CG18480 & 2 & int200_pM37_p0 & FBgn0028518 \\
\hline CR02345 & $\mathrm{cbs}$ & 2 & int200_pM37_p0 & FBgn0086757 \\
\hline CR02346 & Obp58b & 2 & int200_pM37_p0 & FBgn0034768 \\
\hline CR02348 & Tsp42Eq & 2 & int200_pM37_p0 & FBgn0033138 \\
\hline CR02349 & CG15483 & 2 & int200_pM37_p0 & FBgn0032457 \\
\hline CR02351 & CG18301 & 2 & int200_pM37_p0 & FBgn0032265 \\
\hline CR02352 & Tsp39D & 2 & int200_pM37_p0 & FBgn0032943 \\
\hline CR02354 & CG5096 & 2 & int200_pM37_p0 & FBgn0032235 \\
\hline CR02355 & rho-5 & 2 & int200_pM37_p0 & FBgn0041723 \\
\hline CR02357 & CG8407 & 2 & int200_pM37_p0 & FBgn0033687 \\
\hline CR02358 & CG16974 & 2 & int200_pM37_p0 & FBgn0032479 \\
\hline CR02359 & Tsp42Ei & 2 & int200_pM37_p0 & FBgn0033130 \\
\hline CR02360 & Or49b & 2 & int200_pM37_p0 & FBgn0028963 \\
\hline CR02361 & lid & 2 & int200_pM37_p0 & FBgn0031759 \\
\hline CR02362 & CG30043 & 2 & int200_pM37_p0 & FBgn0050043 \\
\hline CR02363 & CG13160 & 2 & int200_pM37_p0 & FBgn0033720 \\
\hline CR02365 & stil & 2 & int200_pM37_p0 & FBgn0003527 \\
\hline CR02367 & $\mathrm{I}(2) 05714$ & 2 & int200_pM37_p0 & FBgn0010607 \\
\hline CR02368 & CG30499 & 2 & int200_pM37_p0 & FBgn0050499 \\
\hline CR02369 & CG1360 & 2 & int200_pM37_p0 & FBgn0033195 \\
\hline CR02370 & Incenp & 2 & int200_pM37_p0 & FBgn0260991 \\
\hline CR02371 & CG9426 & 2 & int200_pM37_p0 & FBgn0032485 \\
\hline CR02374 & Gr28a & 2 & int200_pM37_p0 & FBgn0041247 \\
\hline CR02375 & Odc2 & 2 & int200_pM37_p0 & FBgn0013308 \\
\hline CR02376 & dila & 2 & int200_pM37_p0 & FBgn0033447 \\
\hline CR02378 & CG8738 & 2 & int200_pM37_p0 & FBgn0033321 \\
\hline CR02380 & Alp2 & 2 & int200_pM37_p0 & FBgn0283480 \\
\hline CR02383 & CG4670 & 2 & int200_pM37_p0 & FBgn0033814 \\
\hline CR02385 & BicC & 2 & int200_pM37_p0 & FBgn0000182 \\
\hline CR02388 & CG4712 & 2 & int200_pM37_p0 & FBgn0033818 \\
\hline CR02393 & Rab3 & 2 & int200_pM37_p0 & FBgn0005586 \\
\hline CR02394 & mahj & 2 & int200_pM37_p0 & FBgn0034641 \\
\hline CR02397 & DnaJ-H & 2 & int200_pM37_p0 & FBgn0032474 \\
\hline CR02398 & Tsp42Er & 2 & int200_pM37_p0 & FBgn0033139 \\
\hline CR02399 & Tsp42Eo & 2 & int200_pM37_p1 & FBgn0033136 \\
\hline CR02400 & CG7222 & 2 & int200_pM37_p1 & FBgn0033551 \\
\hline CR02407 & Orcokinin & 2 & int200_pM37_p1 & FBgn0034935 \\
\hline CR02416 & CG30087 & 2 & int200_pM37_p1 & FBgn0050087 \\
\hline CR02419 & Syx6 & 2 & int200_pM37_p1 & FBgn0037084 \\
\hline CR02420 & $\mathrm{I}(2) 03659$ & 2 & int200_pM37_p1 & FBgn0010549 \\
\hline CR02424 & Idgf6 & 2 & int200_pM37_p1 & FBgn0013763 \\
\hline CR02425 & CG31706 & 2 & int200_pM37_p1 & FBgn0051706 \\
\hline CR02427 & emb & 2 & int200_pM37_p1 & FBgn0020497 \\
\hline CR02428 & MED15 & 2 & int200_pM37_p1 & FBgn0027592 \\
\hline CR02429 & CG14401 & 2 & int200_pM37_p1 & FBgn0032900 \\
\hline CR02431 & CG10764 & 2 & int200_pM37_p1 & FBgn0034221 \\
\hline
\end{tabular}


bioRxiv preprint doi: https://doi.org/10.1101/2021.12.24.474112; this version posted December $24,2021$. The copyright holder for this preprint (which was not certified by peer review) is the author/funder, who has granted bioRxiv a license to display the preprint in perpetuity. It is made available under aCC-BY 4.0 International license.

\begin{tabular}{|c|c|c|c|c|}
\hline CR02432 & cnk & 2 & int200_pM37_p1 & FBgn0286070 \\
\hline CR02433 & RhoGAP54D & 2 & int200_pM37_p1 & FBgn0034249 \\
\hline CR02437 & CG10621 & 2 & int200_pM37_p1 & FBgn0032726 \\
\hline CR02438 & CG6967 & 2 & int200_pM37_p1 & FBgn0034187 \\
\hline CR02442 & CG42697 & 2 & int200_pM37_p1 & FBgn0261587 \\
\hline CR02443 & CG33296 & 2 & int200_pM37_p1 & FBgn0053296 \\
\hline CR02444 & spict & 2 & int200_pM37_p1 & FBgn0032451 \\
\hline CR02445 & Idgf3 & 2 & int200_pM37_p1 & FBgn0020414 \\
\hline CR02446 & Sec71 & 2 & int200_pM37_p1 & FBgn0028538 \\
\hline CR02448 & hoe2 & 2 & int200_pM37_p1 & FBgn0031649 \\
\hline CR02450 & CG13793 & 2 & int200_pM37_p1 & FBgn0031935 \\
\hline CR02451 & CG12910 & 2 & int200_pM37_p1 & FBgn0033502 \\
\hline CR02453 & CG13437 & 2 & int200_pM37_p1 & FBgn0034541 \\
\hline CR02454 & CG42319 & 2 & int200_pM37_p1 & FBgn0259219 \\
\hline CR02458 & CG31784 & 2 & int200_pM37_p1 & FBgn0051784 \\
\hline CR02459 & CG30371 & 2 & int200_pM37_p1 & FBgn0050371 \\
\hline CR02462 & CG8353 & 2 & int200_pM37_p1 & FBgn0032002 \\
\hline CR02464 & CG43244 & 2 & int200_pM37_p1 & FBgn0262889 \\
\hline CR02467 & CG5421 & 2 & int200_pM37_p1 & FBgn0032434 \\
\hline CR02468 & CG17760 & 2 & int200_pM37_p1 & FBgn0033756 \\
\hline CR02470 & Ugt36A1 & 2 & int200_pM37_p1 & FBgn0015663 \\
\hline CR02475 & CG14352 & 2 & int200_pM37_p1 & FBgn0031351 \\
\hline CR02476 & CG30172 & 2 & int200_pM37_p2 & FBgn0050172 \\
\hline CR02477 & CG17047 & 2 & int200_pM37_p2 & FBgn0033827 \\
\hline CR02479 & Myd88 & 2 & int200_pM37_p2 & FBgn0033402 \\
\hline CR02480 & CG16799 & 2 & int200_pM37_p2 & FBgn0034538 \\
\hline CR02481 & pgant3 & 2 & int200_pM37_p2 & FBgn0027558 \\
\hline CR02482 & CG5867 & 2 & int200_pM37_p2 & FBgn0027586 \\
\hline CR02484 & sha & 2 & int200_pM37_p2 & FBgn0003382 \\
\hline CR02486 & CG3294 & 2 & int200_pM37_p2 & FBgn0031628 \\
\hline CR02488 & stc & 2 & int200_pM37_p2 & FBgn0001978 \\
\hline CR02490 & Zir & 2 & int200_pM37_p2 & FBgn0031216 \\
\hline CR02491 & CG9270 & 2 & int200_pM37_p2 & FBgn0032908 \\
\hline CR02492 & DUBAI & 2 & int200_pM37_p2 & FBgn0033738 \\
\hline CR02493 & GalNAc-T1 & 2 & int200_pM37_p2 & FBgn0034025 \\
\hline CR02494 & Or33b & 2 & int200_pM37_p2 & FBgn0026391 \\
\hline CR02496 & Or42a & 2 & int200_pM37_p2 & FBgn0033041 \\
\hline CR02497 & Tep1 & 2 & int200_pM37_p2 & FBgn0041183 \\
\hline CR02499 & CG15153 & 2 & int200_pM37_p2 & FBgn0032663 \\
\hline CR02500 & CG31741 & 2 & int200_pM37_p2 & FBgn0051741 \\
\hline CR02501 & Gr33a & 2 & int200_pM37_p2 & FBgn0032416 \\
\hline CR02502 & CG18063 & 2 & int200_pM37_p2 & FBgn0028856 \\
\hline CR02504 & CG15118 & 2 & int200_pM37_p0 & FBgn0034418 \\
\hline CR02505 & CG9515 & 2 & int200_pM37_p0 & FBgn0032077 \\
\hline CR02506 & List & 2 & int200_pM37_p0 & FBgn0034381 \\
\hline CR02509 & crq & 2 & int200_pM37_p0 & FBgn0015924 \\
\hline CR02510 & CG7115 & 2 & int200_pM37_p0 & FBgn0027515 \\
\hline CR02511 & scat & 2 & int200_pM37_p0 & FBgn0011232 \\
\hline
\end{tabular}




\begin{tabular}{|c|c|c|c|c|}
\hline CR02514 & NimB1 & 2 & int200_pM37_p0 & FBgn0027929 \\
\hline CR02517 & SmydA-3 & 2 & int200_pM37_p0 & FBgn0262599 \\
\hline CR02520 & CG30183 & 2 & int200_pM37_p0 & FBgn0050183 \\
\hline CR02521 & Vajk2 & 2 & int200_pM37_p0 & FBgn0032538 \\
\hline CR02522 & CG44836 & 3 & int200_pM37_p0 & FBgn0266099 \\
\hline CR02523 & Madm & 3 & int200_pM37_p0 & FBgn0027497 \\
\hline CR02529 & Dop1R2 & 3 & int200_pM37_p0 & FBgn0266137 \\
\hline CR02532 & CG12880 & 3 & int200_pM37_p0 & FBgn0046258 \\
\hline CR02533 & Ppcs & 3 & int200_pM37_p0 & FBgn0261285 \\
\hline CR02534 & fau & 3 & int200_pM37_p0 & FBgn0266451 \\
\hline CR02535 & Glg1 & 3 & int200_pM37_p0 & FBgn0264561 \\
\hline CR02536 & CG5768 & 3 & int200_pM37_p0 & FBgn0039198 \\
\hline CR02537 & CG34377 & 3 & int200_pM37_p0 & FBgn0263117 \\
\hline CR02542 & dmrt93B & 3 & int200_pM37_p0 & FBgn0038851 \\
\hline CR02543 & Arl8 & 3 & int200_pM37_p0 & FBgn0037551 \\
\hline CR02544 & Ttc26 & 3 & int200_pM37_p0 & FBgn0038358 \\
\hline CR02546 & CG42327 & 3 & int200_pM37_p0 & FBgn0259227 \\
\hline CR02550 & CG11791 & 3 & int200_pM37_p0 & FBgn0039266 \\
\hline CR02554 & CG5196 & 3 & int200_pM37_p0 & FBgn0038039 \\
\hline CR02555 & CG2182 & 3 & int200_pM37_p0 & FBgn0037360 \\
\hline CR02556 & Karybeta3 & 3 & int200_pM37_p0 & FBgn0087013 \\
\hline CR02559 & CG6695 & 3 & int200_pM37_p0 & FBgn0039215 \\
\hline CR02560 & Ada2b & 3 & int200_pM37_p0 & FBgn0037555 \\
\hline CR02562 & Nelf-A & 3 & int200_pM37_p0 & FBgn0038872 \\
\hline CR02565 & Spn85F & 3 & int200_pM37_p0 & FBgn0037772 \\
\hline CR02566 & Root & 3 & int200_pM37_p0 & FBgn0039152 \\
\hline CR02569 & CG15715 & 3 & int200_pM37_p0 & FBgn0036538 \\
\hline CR02573 & unc-13 & 4 & int200_pM37_p0 & FBgn0025726 \\
\hline CR02574 & Arl4 & 4 & int200_pM37_p0 & FBgn0039889 \\
\hline CR02575 & $\mathrm{su}(\mathrm{f})$ & $\mathrm{X}$ & int200_pM37_p0 & FBgn0003559 \\
\hline CR02576 & ogre & $\mathrm{X}$ & int200_pM37_p0 & FBgn0004646 \\
\hline CR02579 & CG2556 & $\mathrm{X}$ & int200_pM37_p0 & FBgn0030396 \\
\hline CR02580 & CG15765 & $\mathrm{X}$ & int200_pM37_p0 & FBgn0029814 \\
\hline CR02581 & CG32547 & $\mathrm{X}$ & int200_pM37_p0 & FBgn0052547 \\
\hline CR02582 & $b r$ & $\mathrm{X}$ & int200_pM37_p0 & FBgn0283451 \\
\hline CR02583 & CG15211 & $\mathrm{X}$ & int200_pM37_p0 & FBgn0030234 \\
\hline CR02584 & CG4928 & $\mathrm{X}$ & int200_pM37_p0 & FBgn0027556 \\
\hline CR02585 & IP3K2 & $\mathrm{X}$ & int200_pM37_p0 & FBgn0283680 \\
\hline CR02586 & rho-4 & $\mathrm{X}$ & int200_pM37_p0 & FBgn0030318 \\
\hline CR02587 & CG9411 & $\mathrm{X}$ & int200_pM37_p0 & FBgn0030569 \\
\hline CR02588 & CG1504 & $\mathrm{X}$ & int200_pM37_p0 & FBgn0031100 \\
\hline CR02589 & CG43901 & $\mathrm{X}$ & int200_pM37_p0 & FBgn0264502 \\
\hline CR02591 & CG32815 & $\mathrm{X}$ & int200_pM37_p0 & FBgn0052815 \\
\hline CR02597 & $t$ & $\mathrm{X}$ & int200_pM37_p0 & FBgn0086367 \\
\hline CR02598 & CG42323 & $\mathrm{X}$ & int200_pM37_p0 & FBgn0259223 \\
\hline CR02599 & CG14626 & $\mathrm{X}$ & int200_pM37_p1 & FBgn0040360 \\
\hline CR02602 & Evi5 & $\mathrm{X}$ & int200_pM37_p1 & FBgn0262740 \\
\hline CR02604 & CG14435 & $\mathrm{X}$ & int200_pM37_p1 & FBgn0029911 \\
\hline
\end{tabular}


bioRxiv preprint doi: https://doi.org/10.1101/2021.12.24.474112; this version posted December $24,2021$. The copyright holder for this preprint (which was not certified by peer review) is the author/funder, who has granted bioRxiv a license to display the preprint in perpetuity. It is made available under aCC-BY 4.0 International license.

\begin{tabular}{|c|c|c|c|c|}
\hline CR02605 & Nup153 & $\mathrm{X}$ & int200_pM37_p1 & FBgn0061200 \\
\hline CR02607 & OtopLa & $\mathrm{X}$ & int200_pM37_p1 & FBgn0259994 \\
\hline CR02608 & CG14625 & $\mathrm{X}$ & int200_pM37_p1 & FBgn0040358 \\
\hline CR02609 & CG32536 & $\mathrm{X}$ & int200_pM37_p1 & FBgn0052536 \\
\hline CR02612 & Ucp4A & $\mathrm{X}$ & int200_pM37_p1 & FBgn0030872 \\
\hline CR02615 & inc & $\mathrm{X}$ & int200_pM37_p1 & FBgn0025394 \\
\hline CR02616 & Kap3 & $\mathrm{X}$ & int200_pM37_p1 & FBgn0028421 \\
\hline CR02617 & Ir8a & $\mathrm{X}$ & int200_pM37_p1 & FBgn0052704 \\
\hline CR02621 & par-6 & $\mathrm{X}$ & int200_pM37_p1 & FBgn0026192 \\
\hline CR02624 & CG1636 & $\mathrm{X}$ & int200_pM37_p1 & FBgn0030030 \\
\hline CR02631 & $z f 30 C$ & 2 & int200_pM37_p1 & FBgn0270924 \\
\hline CR02633 & Ten-m & 3 & int200_pM37_p1 & FBgn0004449 \\
\hline CR02634 & nAChRbeta1 & 3 & int200_pM37_p1 & FBgn0000038 \\
\hline CR02670 & CaMKI & 4 & int200_pM37_p1 & FBgn0016126 \\
\hline CR02671 & rdgA & $\mathrm{X}$ & int200_pM37_p1 & FBgn0261549 \\
\hline CR02672 & CG44422 & $\mathrm{X}$ & int200_pM37_p1 & FBgn0265595 \\
\hline CR02673 & CG42340 & $\mathrm{X}$ & int200_pM37_p1 & FBgn0259242 \\
\hline CR02676 & CG43740 & $\mathrm{X}$ & int200_pM37_p1 & FBgn0263997 \\
\hline CR02677 & CG33181 & $\mathrm{X}$ & int200_pM37_p1 & FBgn0053181 \\
\hline CR02679 & CG32647 & $\mathrm{X}$ & int200_pM37_p1 & FBgn0052647 \\
\hline CR02680 & Hers & $\mathrm{X}$ & int200_pM37_p1 & FBgn0052529 \\
\hline CR02681 & CG1695 & $\mathrm{X}$ & int200_pM37_p2 & FBgn0031116 \\
\hline CR02684 & CG42541 & $\mathrm{X}$ & int200_pM37_p2 & FBgn0260658 \\
\hline CR02687 & CG2256 & $\mathrm{X}$ & int200_pM37_p2 & FBgn0029995 \\
\hline CR02693 & inaF-D & $\mathrm{X}$ & int200_pM37_p1 & FBgn0260812 \\
\hline CR02694 & CG17162 & $\mathrm{X}$ & int200_pM37_p2 & FBgn0039944 \\
\hline CR02697 & b6 & $\mathrm{X}$ & int200_pM37_p0 & FBgn0024897 \\
\hline CR02699 & $\mathrm{Hk}$ & $\mathrm{X}$ & int200_pM37_p0 & FBgn0263220 \\
\hline CR02701 & CG8568 & $\mathrm{X}$ & int200_pM37_p1 & FBgn0030841 \\
\hline CR02702 & CG12065 & $\mathrm{X}$ & int200_pM37_p1 & FBgn0030052 \\
\hline CR02705 & CG1545 & $\mathrm{X}$ & int200_pM37_p1 & FBgn0030259 \\
\hline CR02707 & dmrt11E & $\mathrm{X}$ & int200_pM37_p1 & FBgn0030477 \\
\hline CR02708 & CG1532 & $\mathrm{X}$ & int200_pM37_p1 & FBgn0031143 \\
\hline CR02709 & CG7332 & $\mathrm{X}$ & int200_pM37_p1 & FBgn0030973 \\
\hline CR02711 & CG13012 & $\mathrm{X}$ & int200_pM37_p2 & FBgn0030769 \\
\hline CR02713 & CG4404 & $\mathrm{X}$ & int200_pM37_p0 & FBgn0030432 \\
\hline CR02721 & CG8908 & 2 & int200_pM37_p0 & FBgn0034493 \\
\hline CR02722 & snama & 2 & int200_pM37_p0 & FBgn0086129 \\
\hline CR02723 & TwdIE & 2 & int200_pM37_p0 & FBgn0031957 \\
\hline CR02745 & hiw & $\mathrm{X}$ & int200_pM37_p1 & FBgn0030600 \\
\hline CR02779 & Slip1 & 4 & int200_pM37_p1 & FBgn0024728 \\
\hline CR02793 & CG11360 & 4 & int200_pM37_p1 & FBgn0039920 \\
\hline CR02798 & mAChR-A & 2 & int200_pM37_p1 & FBgn0000037 \\
\hline CR70009 & lip3 & 3 & int200_pM37_p0 & FBgn0023495 \\
\hline CR70012 & tau & 3 & int200_pM37_p0 & FBgn0266579 \\
\hline CR70028 & CtsB1 & $\mathrm{X}$ & int200_pM37_p0 & FBgn0030521 \\
\hline CR70032 & ppt1 & $x$ & int200_pM37_p2 & FBgn0030057 \\
\hline CR70034 & spt6 & $\mathrm{X}$ & int200_pM37_p1 & FBgn0028982 \\
\hline
\end{tabular}




\begin{tabular}{|l|l|c|l|l|}
\hline CR70035 & Tob & X & int200_pM37_p0 & FBgn0028397 \\
\hline CR70041 & CG16935 & 2 & int200_pM37_p1 & FBgn0033883 \\
\hline CR70044 & iPLA2-VIA & 3 & int200_pM37_p1 & FBgn0036053 \\
\hline CR70029 & dsb & 3 & int200_pM37_p2 & FBgn0035290 \\
\hline CR70033 & prt & 3 & int200_pM37_p1 & FBgn0043005 \\
\hline CR70037 & aux & 3 & int200_pM37_p1 & FBgn0037218 \\
\hline CR70036 & anne & 4 & int200_pM37_p1 & FBgn0052000 \\
\hline CR70070 & lgs & 4 & int200_pM37_p1 & FBgn0039907 \\
\hline CR70074 & mim & 2 & int200_pM37_p0 & FBgn0053558 \\
\hline CR70088 & csp & 3 & int200_pM37_p2 & FBgn0004179 \\
\hline CR70089 & Cdase & 3 & int200_pM37_p0 & FBgn0039774 \\
\hline CR70071 & CG3226 & X & int200_pM37_p2 & FBgn0029882 \\
\hline CR70077 & GLS & 2 & int200_pM37_p2 & FBgn0261625 \\
\hline CR70087 & CG7236 & 2 & int200_pM37_p2 & FBgn0031730 \\
\hline CR70048 & Trp-Gamma & 2 & int200_TGEM_p2 & FBgn0032593 \\
\hline
\end{tabular}




int200_KozakGAL4 strategy
\begin{tabular}{|l|l|c|c|l|}
\hline \multicolumn{1}{|c}{ CR } & Gene & Chr. & Donor DNA & FlyBase FBgn \\
\hline CR70003 & CG14044 & 2 & int200_pM37_kG4 & FBgn0031650 \\
\hline CR70002 & CG15728 & X & int200_pM37_kG4 & FBgn0030409 \\
\hline CR70005 & CG16787 & 2 & int200_pM37_kG4 & FBgn0034940 \\
\hline CR70008 & CG8027 & 2 & int200_pM37_kG4 & FBgn0033392 \\
\hline CR70013 & Ast-C & 2 & int200_pM37_kG4 & FBgn0032336 \\
\hline CR70011 & CG16979 & 3 & int200_pM37_kG4 & FBgn0036512 \\
\hline CR70014 & CG13737 & 3 & int200_pM37_kG4 & FBgn0036382 \\
\hline CR70038 & CG3376 & 2 & int200_pM37_kG4 & FBgn0034997 \\
\hline CR70045 & oli & 2 & int200_pM37_kG4 & FBgn0032651 \\
\hline CR70046 & SK1 & X & int200_pM37_kG4 & FBgn0030300 \\
\hline CR70043 & intS11 & 3 & int200_pM37_kG4 & FBgn0039691 \\
\hline CR70020 & B-Glu & 3 & int200_pM37_kG4 & FBgn0270927 \\
\hline CR70040 & CG8320 & 2 & int200_pM37_kG4 & FBgn0034059 \\
\hline CR70022 & CG14457 & 3 & int200_pM37_kG4 & FBgn0037174 \\
\hline CR70053 & pngl & 2 & int200_pM37_kG4 & FBgn0033050 \\
\hline CR70068 & Bap111 & X & int200_pM37_kG4 & FBgn0030093 \\
\hline CR70069 & Bap60 & X & int200_pM37_kG4 & FBgn0025463 \\
\hline CR70050 & Vkor & 2 & int200_pM37_kG4 & FBgn0053544 \\
\hline CR70079 & Hip1 & 3 & int200_pM37_kG4 & FBgn0036309 \\
\hline CR70080 & PIGA & 2 & int200_pM37_kG4 & FBgn0288203 \\
\hline CR70082 & elo68Beta & 3 & int200_pM37_kG4 & FBgn0036128 \\
\hline CR70083 & CG15744 & X & int200_pM37_kG4 & FBgn0030466 \\
\hline
\end{tabular}


gRNA_int200_T2AGAL4 strategy

\begin{tabular}{|c|c|c|c|c|}
\hline$\underline{\mathrm{CR}}$ & Gene & Chr. & Donor DNA & FlyBase FBgn \\
\hline CR70092 & ara & 3 & |gRNA_int200_pM37_p0 & FBgn0015904 \\
\hline CR70094 & lace & 2 & gRNA_int200_pM37_p0 & FBgn0002524 \\
\hline CR70095 & Pex23 & 3 & gRNA_int200_pM37_p2 & FBgn0052226 \\
\hline CR70096 & DIP- $\lambda$ & 2 & gRNA_int200_pM37_p0 & FBgn0267428 \\
\hline CR70097 & fwe & 3 & gRNA_int200_pM37_p1 & FBgn0261722 \\
\hline CR70098 & salr & 2 & gRNA_int200_pM37_p0 & FBgn0000287 \\
\hline CR70099 & CG15528 & 3 & gRNA_int200_pM37_p2 & FBgn0039742 \\
\hline CR70107 & dmrt99B & 3 & gRNA_int200_pM37_p0 & FBgn0039683 \\
\hline CR70108 & Lsd2 & $\bar{x}$ & gRNA_int200_pM37_p1 & FBgn0030608 \\
\hline CR70110 & SREBP & 3 & gRNA_int200_pM37_p0 & FBgn0261283 \\
\hline CR70114 & RhoGAP68F & 3 & gRNA_int200_pM37_p1 & FBgn0036257 \\
\hline CR70115 & ifc & 2 & gRNA_int200_pM37_p2 & FBgn0001941 \\
\hline CR70118 & KrT95D & 3 & gRNA_int200_pM37_p0 & FBgn0020647 \\
\hline CR70121 & Mfe2 & $\mathrm{x}$ & |gRNA_int200_pM37_p1 & FBgn0 \\
\hline CR70122 & Vps 13 & 2 & gRNA_int200_pM37_p1 & FBgn0033194 \\
\hline CR70125 & CG6967 & 2 & gRNA_int200_pM37_p1 & FBgn0034187 \\
\hline CR70127 & CG33090 & 2 & gRNA_int200_pM37_p2 & FBgn0028916 \\
\hline CR70133 & $\mathrm{ec}$ & $x$ & gRNA_int200_pM37_p0 & FBgnO \\
\hline CR70135 & $\mathrm{srl}$ & 3 & gRNA_int200_pM37_p2 & FBgnO \\
\hline CR70136 & Root & 3 & gRNA_int200_pM37_p0 & FBgn0039152 \\
\hline CR70137 & Su(z)12 & 3 & gRNA_int200_pM37_p0 & FBgn0020887 \\
\hline CR70140 & $\mathrm{cnn}$ & 2 & gRNA_int200_pM37_p1 & FBgn0013765 \\
\hline CR70144 & AdenoK & 3 & |gRNA_int200_pM37_p0 & FBgn0036337 \\
\hline CR70145 & CG8199 & 3 & gRNA_int200_pM37_p2 & FBgn0037709 \\
\hline CR70149 & CG5044 & 3 & gRNA_int200_pM37_p0 & FBgnOC \\
\hline CR70150 & sub & 2 & gRNA_int200_pM37_p0 & FBgn0 \\
\hline CR70151 & Tusp & 3 & gRNA_int200_pM37_p0 & FBgn0039530 \\
\hline CR70152 & FarO & $\mathrm{X}$ & gRNA_int200_pM37_p2 & FBgn0023550 \\
\hline CR70157 & CG14736 & 3 & gRNA_int200_pM37_p1 & FBgn0037986 \\
\hline CR70162 & CG4612 & 2 & |gRNA_int200_pM37_p0 & FBgn0035016 \\
\hline CR70165 & DNApolalpha & 3 & gRNA_int200_pM37_p1 & FBgn0259113 \\
\hline CR70166 & mav & 4 & gRNA_int200_pM37_p0 & FBgn0039914 \\
\hline CR70167 & rhea & 3 & gRNA_int200_pM37_p0 & FBgn0260442 \\
\hline CR70168 & PK2-R1 & 3 & gRNA_int200_pM37_p2 & FBgn0038140 \\
\hline CR70173 & LKRSDH & 2 & gRNA_int200_pM37_p0 & FBgn0286198 \\
\hline CR70174 & CG7414 & 3 & gRNA_int200_pM37_p1 & FBgn0037135 \\
\hline CR70177 & CG12268 & 3 & gRNA_int200_pM37_p0 & FBgn0039131 \\
\hline CR70178 & UQCR-c1 & 3 & gRNA_int200_pM37_p0 & FBgn0038271 \\
\hline CR70179 & wal & 2 & |gRNA_int200_pM37_p0 & FBgn0010516 \\
\hline CR70187 & CG18155 & $\bar{x}$ & gRNA_int200_pM37_p1 & FBgn0029945 \\
\hline CR70188 & CG33253 & $x$ & gRNA_int200_pM37_p1 & FBgn0030992 \\
\hline CR70189 & Grasp65 & 3 & gRNA_int200_pM37_p1 & FBgn0036919 \\
\hline CR70190 & $\operatorname{IntS11}$ & 3 & gRNA_int200_pM37_p1 & FBgn0039691 \\
\hline CR70191 & Mrtf & 3 & gRNA_int200_pM37_p1 & FBgn0052296 \\
\hline CR70192 & pAbp & 2 & gRNA_int200_pM37_p1 & FBgn0265297 \\
\hline CR70196 & rin & 3 & gRNA_int200_pM37_p1 & FBgn0015778 \\
\hline
\end{tabular}


bioRxiv preprint doi: https://doi.org/10.1101/2021.12.24.474112; this version posted December $24,2021$. The copyright holder for this preprint (which was not certified by peer review) is the author/funder, who has granted bioRxiv a license to display the preprint in perpetuity. It is made available under aCC-BY 4.0 International license.

\begin{tabular}{|c|c|c|c|c|}
\hline CR70197 & wat & 3 & gRNA_int200_pM37_p1 & FBgn0039620 \\
\hline CR70199 & CG5599 & $x$ & gRNA_int200_pM37_p0 & FBgn0030612 \\
\hline CR70200 & CG17168 & $\mathrm{X}$ & gRNA_int200_pM37_p2 & FBgn0039943 \\
\hline CR70201 & Edem2 & 2 & gRNA_int200_pM37_p2 & FBgn0032480 \\
\hline CR70202 & GM130 & 2 & gRNA_int200_pM37_p0 & FBgn0034697 \\
\hline CR70205 & nocte & $\mathrm{X}$ & gRNA_int200_pM37_p0 & FBgn0261710 \\
\hline CR70207 & pex1 & 3 & gRNA_int200_pM37_p0 & FBgn0013563 \\
\hline CR70208 & Prx6005 & 2 & gRNA_int200_pM37_p2 & FBgn0031479 \\
\hline CR70209 & Rlip & 3 & gRNA_int200_pM37_p2 & FBgn0026056 \\
\hline CR70210 & roe & 2 & gRNA_int200_pM37_p0 & FBgn0267337 \\
\hline CR70212 & vha68-2 & 2 & gRNA_int200_pM37_p0 & FBgn0263598 \\
\hline CR70213 & $\mathrm{sp1}$ & $x$ & gRNA_int200_pM37_p2 & FBgn0020378 \\
\hline CR70216 & $\mathrm{br}$ & $\mathrm{X}$ & gRNA_int200_pM37_p0 & FBgn0283451 \\
\hline CR70218 & Axn & 3 & gRNA_int200_pM37_p2 & FBgn0026597 \\
\hline CR70219 & Lpr1 & 3 & gRNA_int200_pM37_p0 & FBgn0066101 \\
\hline CR70220 & Lpr2 & 3 & gRNA_int200_pM37_p0 & FBgn0051092 \\
\hline CR70225 & Osi2 & 3 & gRNA_int200_pM37_p2 & FBgn0037410 \\
\hline CR70227 & wap & $\mathrm{X}$ & gRNA_int200_pM37_p2 & FBgn0266848 \\
\hline CR70229 & LARP4B & 3 & gRNA_int200_pM37_p0 & FBgn0283788 \\
\hline CR70231 & DIP- $\beta$ & $X$ & gRNA_int200_pM37_p1 & FBgn0259245 \\
\hline CR70233 & cac & $\mathrm{X}$ & gRNA_int200_pM37_p1 & FBgn0263111 \\
\hline CR70241 & CG7956 & 3 & gRNA_int200_pM37_p1 & FBgn0038890 \\
\hline CR70242 & Iola & 2 & gRNA_int200_pM37_p1 & FBgn0283521 \\
\hline CR70243 & sli & 2 & gRNA_int200_pM37_p1 & FBgn0264089 \\
\hline CR70244 & $\mathrm{DI}$ & 3 & gRNA_int200_pM37_p0 & FBgn0000463 \\
\hline CR70246 & Csp & 3 & gRNA_int200_pM37_p0 & FBgn0004179 \\
\hline CR70249 & MFS10 & $\mathrm{X}$ & gRNA_int200_pM37_p1 & FBgn0030452 \\
\hline CR70251 & elF4B & 3 & gRNA_int200_pM37_p1 & FBgn0020660 \\
\hline CR70256 & Pis & $x$ & gRNA_int200_pM37_p1 & FBgn0030670 \\
\hline CR70258 & Rpt3 & $x$ & gRNA_int200_pM37_p0 & FBgn0028686 \\
\hline CR70260 & CG10907 & 3 & gRNA_int200_pM37_p2 & FBgn0036207 \\
\hline CR70261 & tdc2 & 2 & gRNA_int200_pM37_p2 & FBgn0050446 \\
\hline CR70263 & Gdap1 & 3 & gRNA_int200_pM37_p0 & FBgn0035587 \\
\hline CR70265 & golgin245 & 2 & gRNA_int200_pM37_p0 & FBgn0034854 \\
\hline CR70266 & ThrRS & 2 & gRNA_int200_pM37_p1 & FBgn0027081 \\
\hline CR70268 & CG10137 & 2 & gRNA_int200_pM37_p2 & FBgn0032800 \\
\hline CR70269 & CG17739 & 2 & gRNA_int200_pM37_p1 & FBgn0033710 \\
\hline CR70281 & CrebA & 3 & gRNA_int200_pM37_p0 & FBgn0004396 \\
\hline CR70282 & $g \mathrm{gl}$ & 3 & gRNA_int200_pM37_p0 & FBgn0004618 \\
\hline CR70284 & ND-49 & 4 & gRNA_int200_pM37_p1 & FBgn0039909 \\
\hline CR70287 & $\mathrm{kis}$ & 2 & gRNA_int200_pM37_p0 & FBgn0266557 \\
\hline CR70289 & erm & 2 & gRNA_int200_pM37_p0 & FBgn0031375 \\
\hline CR70296 & Yif1 & 3 & gRNA_int200_pM37_p1 & FBgn0039450 \\
\hline CR70298 & RhoGAP100 & 3 & gRNA_int200_pM37_p1 & FBgn0039883 \\
\hline CR70299 & gsc & 2 & gRNA_int200_pM37_p0 & FBgn0010323 \\
\hline CR70312 & CG7744 & 2 & gRNA_int200_pM37_p2 & FBgn0034447 \\
\hline CR70314 & Itgbn & 2 & gRNA_int200_pM37_p1 & FBgn0010395 \\
\hline CR70324 & Tim10 & 2 & gRNA_int200_pM37_p1 & FBgn0027360 \\
\hline
\end{tabular}


bioRxiv preprint doi: https://doi.org/10.1101/2021.12.24.474112; this version posted December 24, 2021. The copyright holder for this

preprint (which was not certified by peer review) is the author/funder, who has granted bioRxiv a license to display the preprint in perpetuity. It is made available under aCC-BY 4.0 International license.

\begin{tabular}{|l|l|c|l|l}
\hline CR70329 & stai & 2 & gRNA_int200_pM37_p1 & FBgn0266521
\end{tabular}


2XgRNA_int200_KozakGAL4 strategy

\begin{tabular}{|c|c|c|c|c|}
\hline$\underline{\mathrm{CR}}$ & Gene & Chr. & Donor DNA & FlyBase FBgn \\
\hline CR70091 & CG7423 & $\mathrm{X}$ & 2XgRNA_int200_pM37_kG4 & FBgn0030982 \\
\hline CR70093 & CG7504 & 3 & 2XgRNA_int200_pM37_kG4 & FBgn0035842 \\
\hline CR70104 & CG14795 & $x$ & 2XgRNA_int200_pM37_kG4 & FBgn0025393 \\
\hline CR70106 & CG33310 & 2 & 2XgRNA_int200_pM37_kG4 & FBgn0053310 \\
\hline CR70109 & PHGPX & 3 & 2XgRNA_int200_pM37_kG4 & FBgn0035438 \\
\hline CR70111 & Wdr37 & 3 & 2XgRNA_int200_pM37_kG4 & FBgn0038617 \\
\hline CR70112 & ZnT49B & 2 & 2XgRNA_int200_pM37_kG4 & FBgn0033762 \\
\hline CR70116 & CG14644 & 3 & 2XgRNA_int200_pM37_kG4 & FBgn0250821 \\
\hline CR70119 & CG10947 & 2 & 2XgRNA_int200_pM37_kG4 & FBgn0032857 \\
\hline CR70120 & Usp7 & $x$ & 2XgRNA_int200_pM37_kG4 & FBgn0030366 \\
\hline CR70124 & Vps13B & 3 & 2XgRNA_int200_pM37_kG4 & FBgn0039727 \\
\hline CR70130 & mAChR-C & $x$ & 2XgRNA_int200_pM37_kG4 & FBgn0029909 \\
\hline CR70131 & CG34293 & 3 & 2XgRNA_int200_pM37_kG4 & FBgn0085322 \\
\hline CR70139 & wfs 1 & 3 & 2XgRNA_int200_pM37_kG4 & FBgn0039003 \\
\hline CR70142 & $\mathrm{Ct} 12$ & 3 & 2XgRNA_int200_pM37_kG4 & FBgn0039637 \\
\hline CR70143 & CG8778 & 2 & 2XgRNA_int200_pM37_kG4 & FBgn0033761 \\
\hline CR70146 & GlcT & 2 & 2XgRNA_int200_pM37_kG4 & FBgn0067102 \\
\hline CR70147 & CG11103 & $\mathrm{x}$ & 2XgRNA_int200_pM37_kG4 & FBgn0030522 \\
\hline CR70148 & CG30022 & 2 & 2XgRNA_int200_pM37_kG4 & FBgn0050022 \\
\hline CR70153 & Ufm1 & 2 & 2XgRNA_int200_pM37_kG4 & FBgn0085220 \\
\hline CR70154 & CG8646 & 2 & 2XgRNA_int200_pM37_kG4 & FBgn0033763 \\
\hline CR70155 & $\operatorname{cln} 3$ & 3 & 2XgRNA_int200_pM37_kG4 & FBgn0036756 \\
\hline CR70156 & CG10104 & 2 & 2XgRNA_int200_pM37_kG4 & FBgn0033933 \\
\hline CR70160 & CG3770 & 2 & 2XgRNA_int200_pM37_kG4 & FBgn0035085 \\
\hline CR70169 & rhea & 3 & 2XgRNA_int200_pM37_kG4 & FBgn0260442 \\
\hline CR70176 & Loxl2 & 2 & 2XgRNA_int200_pM37_kG4 & FBgn0034660 \\
\hline CR70181 & CG2124 & $x$ & 2XgRNA_int200_pM37_kG4 & FBgn0030217 \\
\hline CR70182 & CG4074 & 3 & 2XgRNA_int200_pM37_kG4 & FBgn0037017 \\
\hline CR70183 & CG6638 & 3 & 2XgRNA_int200_pM37_kG4 & FBgn0035911 \\
\hline CR70184 & CG8202 & 3 & 2XgRNA_int200_pM37_kG4 & FBgn0037622 \\
\hline CR70185 & CG9184 & 3 & 2XgRNA_int200_pM37_kG4 & FBgn0035208 \\
\hline CR70186 & CG15093 & 2 & 2XgRNA_int200_pM37_kG4 & FBgn0034390 \\
\hline CR70193 & pex2 & 3 & 2XgRNA_int200_pM37_kG4 & FBgn0035876 \\
\hline CR70194 & pex16 & 3 & 2XgRNA_int200_pM37_kG4 & FBgn0037019 \\
\hline CR70215 & ash1 & 3 & 2XgRNA_int200_pM37_kG4 & FBgn0005386 \\
\hline CR70221 & Snx17 & 2 & 2XgRNA_int200_pM37_kG4 & FBgn0032191 \\
\hline CR70222 & Vps29 & 2 & 2XgRNA_int200_pM37_kG4 & FBgn0031310 \\
\hline CR70223 & AP2-sigma & 3 & 2XgRNA_int200_pM37_kG4 & FBgn0043012 \\
\hline CR70226 & CG7943 & 3 & 2XgRNA_int200_pM37_kG4 & FBgn0039741 \\
\hline CR70237 & Fitm & 3 & 2XgRNA_int200_pM37_kG4 & FBgn0035586 \\
\hline CR70238 & armi & 3 & 2XgRNA_int200_pM37_kG4 & FBgn0041164 \\
\hline CR70245 & CG5254 & $x$ & 2XgRNA_int200_pM37_kG4 & FBgn0040383 \\
\hline CR70247 & Pka-C1 & 2 & 2XgRNA_int200_pM37_kG4 & FBgn0000273 \\
\hline CR70248 & Ufc1 & 2 & 2XgRNA_int200_pM37_kG4 & FBgn0034061 \\
\hline CR70252 & $\mathrm{mtm}$ & 2 & 2XgRNA_int200_pM37_kG4 & FBgn0025742 \\
\hline CR70262 & CG7716 & 3 & 2XgRNA_int200_pM37_kG4 & FBgn0035800 \\
\hline
\end{tabular}




\begin{tabular}{|l|l|c|l|l|}
\hline CR70264 & mib1 & 3 & 2XgRNA_int200_pM37_kG4 & FBgn0263601 \\
\hline CR70267 & shop & X & 2XgRNA_int200_pM37_kG4 & FBgn0030966 \\
\hline CR70270 & GLS & 2 & 2XgRNA_int200_pM37_kG4 & FBgn0261625 \\
\hline CR70271 & CG13051 & 3 & 2XgRNA_int200_pM37_kG4 & FBgn0040799 \\
\hline CR70272 & CG32191 & 3 & 2XgRNA_int200_pM37_kG4 & FBgn0052191 \\
\hline CR70273 & Arc2 & 2 & 2XgRNA_int200_pM37_kG4 & FBgn0033928 \\
\hline CR70274 & SoxN & 2 & 2XgRNA_int200_pM37_kG4 & FBgn0029123 \\
\hline CR70276 & CG10324 & 3 & 2XgRNA_int200_pM37_kG4 & FBgn0038454 \\
\hline CR70278 & CG34247 & 3 & 2XgRNA_int200_pM37_kG4 & FBgn0085276 \\
\hline CR70279 & whip & 2 & 2XgRNA_int200_pM37_kG4 & FBgn0250827 \\
\hline CR70280 & CG7402 & 3 & 2XgRNA_int200_pM37_kG4 & FBgn0036768 \\
\hline CR70283 & Qsox2 & 3 & 2XgRNA_int200_pM37_kG4 & FBgn0038919 \\
\hline CR70290 & CG10073 & 2 & 2XgRNA_int200_pM37_kG4 & FBgn0034440 \\
\hline
\end{tabular}


Supplementary material Primer sequences

Primers for PCR verification of CRIMIC and KozakGAL4 alleles

\begin{tabular}{|l|l|}
\hline KozakGAL4_L_R & ACTCCCAGTTGTTCTTCAGGCACTTGGC \\
\hline KozakGAL4_R_F & GAAAGTATAGGAACTTCTCGCGCTCG \\
\hline \multicolumn{2}{|l|}{} \\
\hline CRIMIC_ch_rev & GCGGAAGAGAGATAAATCGGTTG \\
\hline CRIMIC_ch_for & GTGGTATGGCTGATTATGATCAGAAG \\
\hline
\end{tabular}

Primers to clone WDR37 Knock-in alleles

\begin{tabular}{|c|c|}
\hline \multicolumn{2}{|r|}{ C-Term } \\
\hline dsRed_CTerm_F & AGGCGCAGCTCTGCGAAATCTTAACCCTAGAAAGATAATCATATTGTGACGTACGTTAAAGATAATCATG \\
\hline scDsRed_R & TGTGGGCATCGCTITGGGGATGCTTCTTGTTCGAGAGATTAACCCTAGAAAGATAGTCTGCGTAAAATTGAC \\
\hline sfGFP_Cterm_F & TCGTTCTTCCGAAGGACAATGTGGGAGGTTCCGGTGGAAG \\
\hline sfGFP_CTerm_R & CTCCGCTTCAGTTGCCTTCACTTGTACAGCTCATCCATGCCCAGGGT \\
\hline wdr37_3F_CTerm_F & GCATGGATGAGCTGTACAAGTGAAGGCAACTGAAGCGGAG \\
\hline wdr37_3F_R & GATTATCTTTCTAGGGTTAAGATTTCGCAGAGCTGCGC \\
\hline wdr37_5F_CTerm_R & CTTCCACCGGAACCTCCCACATTGTCCTTCGGAAGAACGATGG \\
\hline wdr37_5F_F & GCGAGTCTGCAACCCCTGAACACTAATTGGATTCCCCCGGGCTCTGCCCACC \\
\hline \multicolumn{2}{|r|}{ internal } \\
\hline sfGFP_int_F & GCATGATTTCAGGAAGGGCGTGGGAGGTTCCGGTGGAAG \\
\hline sfGFP_int_R & AGTTCCTCCTCTGAAGAATGCTCGCCGGAACCTCCG \\
\hline wdr37_3F_int_F & GAAGCGGAGGTTCCGGCGAGCATTCTTCAGAGGAGGAACTGGACG \\
\hline wdr37_5F_int_R & CTTCCACCGGAACCTCCCACGCCCTTCCTGAAAATCATGCAACG \\
\hline \multicolumn{2}{|r|}{ N-Term } \\
\hline sfGFP_Nterm_F & GAGGCGAAAAGAAAAGCATGGTGTCCAAGGGCGAG \\
\hline sfGFP_Nterm_R & GCCCGTGTCTTGCTAGCCTTCTCGCCGGAACCTCCG \\
\hline wdr37_3F_NTerm_F & GAAGCGGAGGTTCCGGCGAGAAGGCTAGCAAGACACGGGCT \\
\hline wdr37_5F_Nterm_R & AGCTCCTCGCCCTTGGACACCATGCTTTCTTTCGCCTCTTGT \\
\hline
\end{tabular}




\section{Supplementary methods Detailed cloning protocol and sequences}

\section{Design of new CRIMIC synthesis constructs:}

\section{Int200 strategy}

Design region:

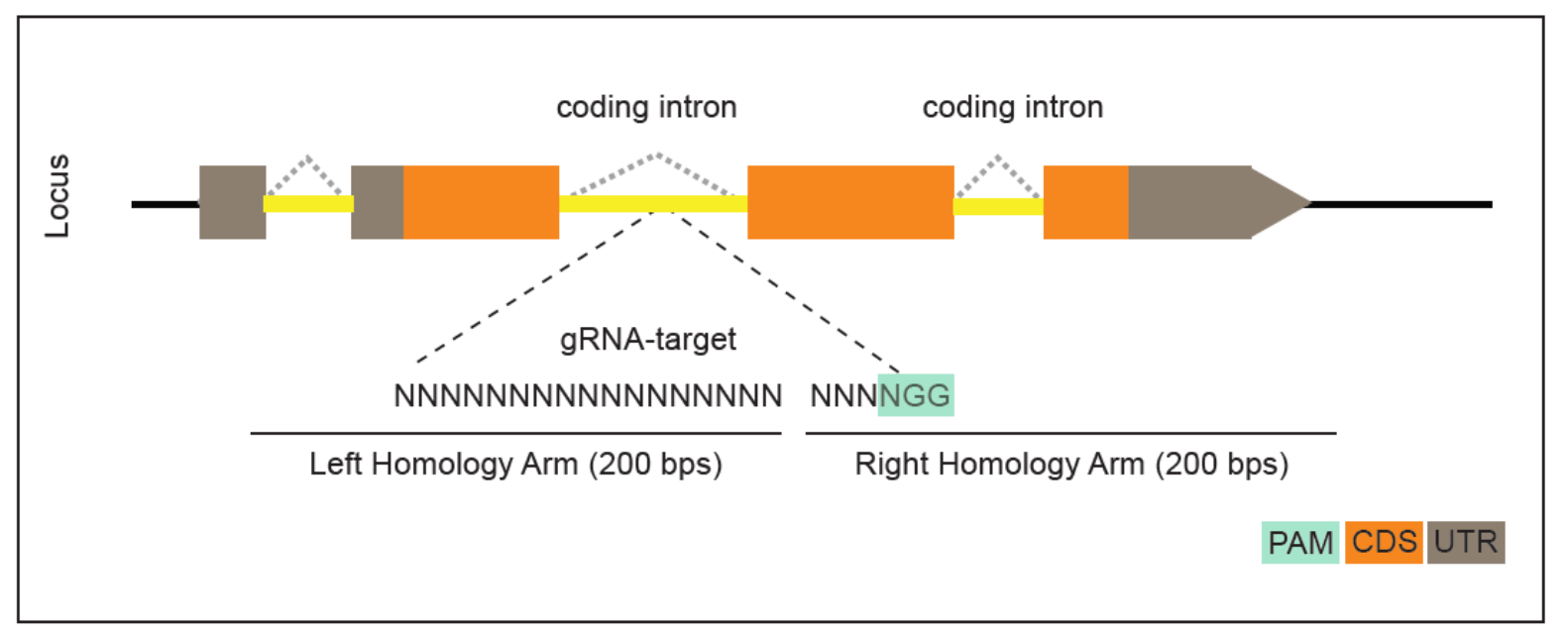

- 200 nucleotides on both sides of the cut site are used for synthesis using the template DNA file (int200_scaffold_Bbsl file, in case the homology arms contain Bbsl sites, Bsal-HF can be used for cloning. In that case int200_scaffold_Bsal scaffold file should be used for ordering the synthesis) Start (0)

- Synthesis is ordered through Genewiz as Value gene synthesis (at 4nM scale). In order notes indicate:

- The constructs should be cloned in pUC57_Kan_gw_OK vector in EcoRV site. This vector has the gRNA1 target sites on either side of EcoRV cut site and hence places the homology arms in between 
gRNA1 cut sites. In the backbone there is a U6:gRNA1 that linearizes the construct in vivo.

- No need to order the glycerol stocks. Lyophilised constructs facilitates the reaction set up.

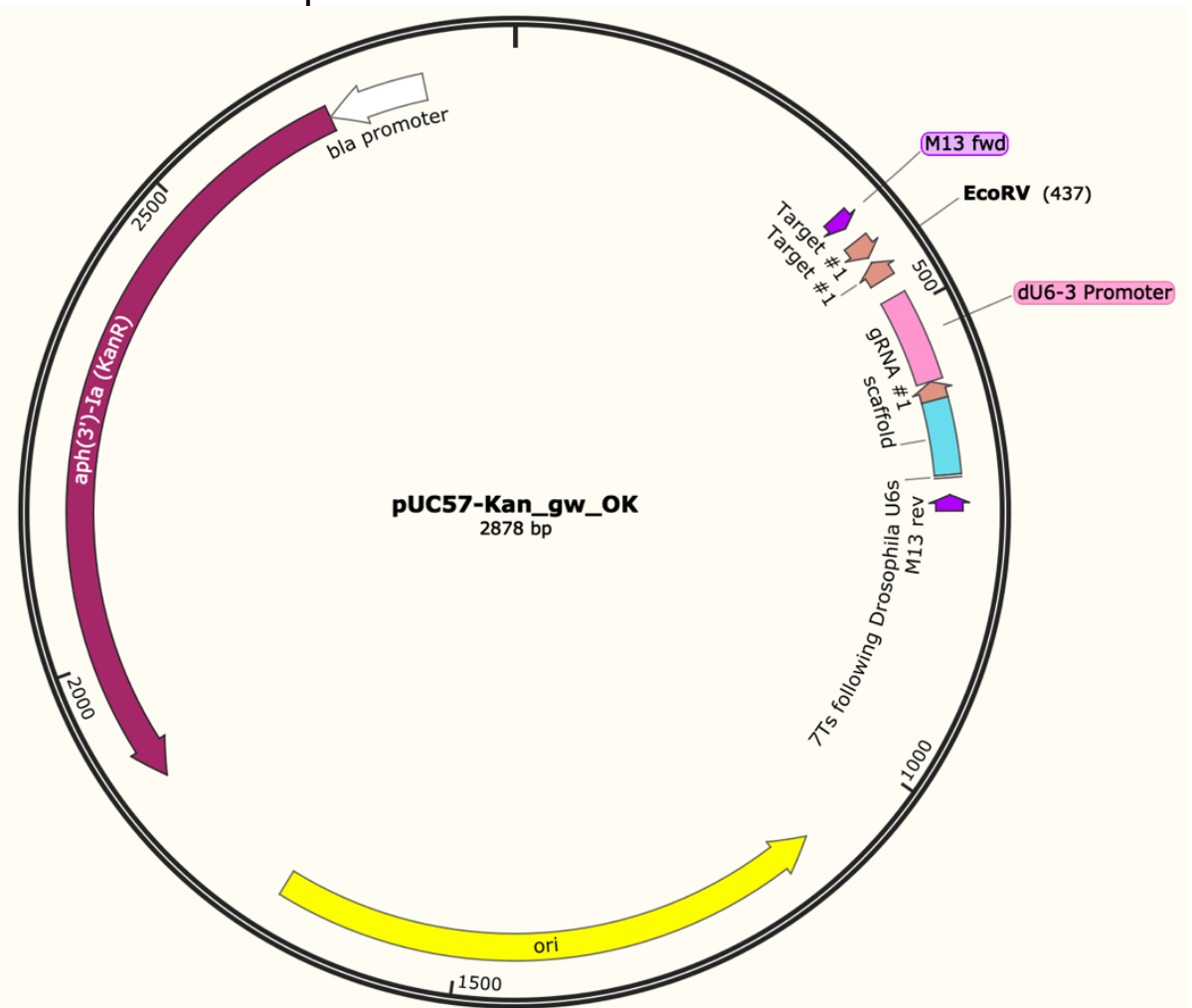

After synthesis the construct looks like below 
bioRxiv preprint doi: https://doi.org/10.1101/2021.12.24.474112; this version posted December 24, 2021. The copyright holder for this preprint (which was not certified by peer review) is the author/funder, who has granted bioRxiv a license to display the preprint in perpetuity. It is made available under aCC-BY 4.0 International license.

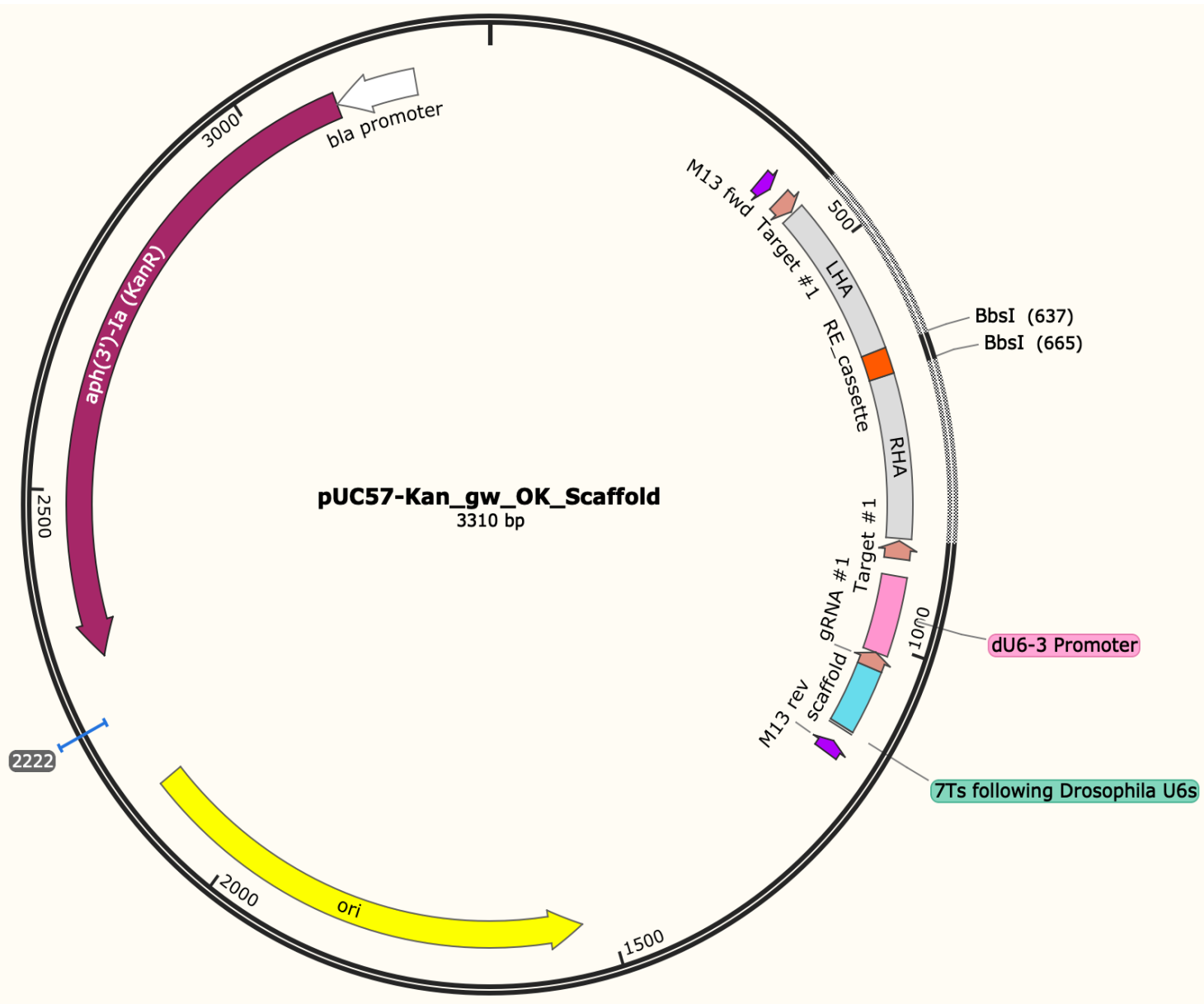

\section{gRNA_int200 strategy \\ Start (0)}

tcccggctggt gcanNNNNNNNNNNNNNNNNNgt t t tagagctagaaatagcaagt taaaataaggctagtccgt tatcaact tgaaaaagtggcaccgagtcggtgcTTTTTTGTAG

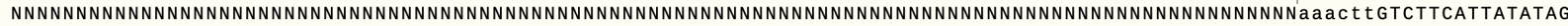
等,

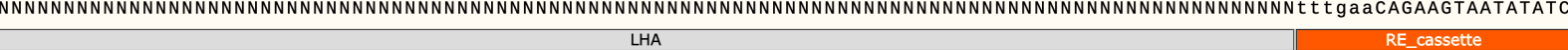

BbsI 
The ordering for gRNA_int200 strategy is similar to int200 strategy with the exception of including the target specific sgRNA sequences in the synthesis order and the synthesis should be conducted on pUC57_Kan_gw_OK2 backbone that contains the rest of the components of the homology donor construct.

For KozakGAL4 constructs 2 sgRNAs are added in the synthesis reaction.

\section{Cloning of constructs:}

-When the constructs arrive, resuspend them in $53 \mu \mathrm{ld} \mathrm{H}_{2} \mathrm{O}$ (Do not resuspend in TE buffer. Genewiz lyophylises the constructs in TE buffer. Resuspending in TE buffer decreases efficacy of downstream cloning applications).

-Select the proper vector of pM37 (correct phase for T2AGAL4 or pM37_KozakGAL4 or pM37_SA_KozakGAL4) with Bbsl-HF (or Bsal-HF if Bsal construct is being used). Set up the reaction (make a master mix for constructs of the same SIC if cloning multiple constructs):

- $1 \mu \mathrm{l} \mathrm{pM37-phase} \mathrm{X}^{*}(290 \mathrm{ng} / \mu \mathrm{l})$ or $1 \mu \mathrm{l}$ pM37_KozakGAL4 $(265 \mathrm{ng} / \mu \mathrm{l})$

- $2.5 \mu \mathrm{l}$ 10X T4 DNA ligase buffer (NEB B0202S)

- $0.5 \mu \mathrm{l}$ T4 DNA ligase (NEB M0202L)

- 1 ul Restriction enzyme (Bbsl_HF (NEB R3733L) or Bsal_HFv2 (NEB R3559L)

- $19 \mu \mathrm{l}$ of $\mathrm{dH}_{2} \mathrm{O}$

\footnotetext{
Start (0) gene_Specific_gRNA_1

tcccggctggtgcanNNNNNNNNNNNNNNNNNNgtt ttagagctagaaatagcaagt taaaataaggctagtccgttatcaacttgaaaaagtggcaccgagtcggt gctaacaaagca agggccgaccacgtnNNNNNNNNNNNNNNNNcaaatctcgatctttatcgttcaatttattccgatcaggcaatagttgaactttttcaccgtggctcagccacgattgtttcgt

ccagtggtctagtggtagaatagtaccctgccacggtacagacccgggt tcgat tcccggctggt gcanNNNNNNNNNNNNNNNNNgt t t tagagctagaaat agcaagt taaaa taa

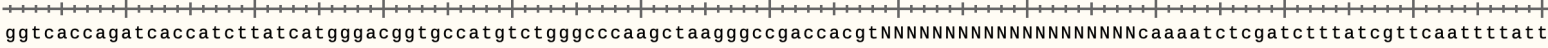

Distribute the master mix in PCR tubes and add $1 \mu$ of reconstituted intermediate plasmid. 
* Phase selection is done by following the reading frame of the gene at the end of the preceding exon. If the last codon in the preceeding exon is complete (NNNintron-NNN), the phase is $\mathrm{p} 0$. If one of the nucleotide of the codon is in the preceeding exon and two are in the following exon ( $N$-intron- $N N)$ the phase is $p 1$. If two nucleotides of the codon is in the preceeding exon and one in the following exon (NN-intron-N) the phase is $\mathrm{p} 2$. It is crucial to select the correct phase using incorrect phase cause a frameshift mutation.

-Incubate the reactions in a Thermocycler:

- $37^{\circ} \mathrm{C} 5$ minutes

- $16^{\circ} \mathrm{C} 5$ minutes

- Go to 130 times

- $65^{\circ} \mathrm{C} 20$ minutes

- $\quad 8^{\circ} \mathrm{C}$ hold

The reactions can be left in the thermocycler overnight.

- An additional digestion step is done to remove self ligating plasmid backbones by adding:

- $19.5 \mu \mathrm{d} \mathrm{dH} \mathrm{H}_{2} \mathrm{O}$

- $5 \mu \mathrm{l}$ 10X Cutsmart buffer

- $0.5 \mu \mathrm{l} \mathrm{Bbsl}$ or Bsal_HFv2 (the enzyme used for the cloning reaction)

The restriction mix can also be prepared as a mastermix and distributed to each sample $25 \mu \mathrm{l} /$ sample. Incukbate the reactions for 30 minutes in $37^{\circ} \mathrm{C}$ incubator or thermocycler.

-Transform to 50 ul chemocompetent DH5-alpha. Selection antibiotic is Kanamycin, hence 1-hour recovery is necessary after heatshock. Plate on LB plates with Kanamycin. Incubate at $37^{\circ} \mathrm{C}$ overnight.

-(Optional) Next day do colony PCR with primers M13F_Long_for CRIMIC_ch_rev

M13F_long_for gacgttgtaaaacgacggccag

CRIMIC_ch_rev gcggaagagagataaatcggttg

I use an autoclaved micropipette tip to pick a colony, touch it on a gridded plate to copy the colony and dip the same pipette tip to PCR mix.

PCR conditions

0.2 ul forward primer

$0.2 \mathrm{ul}$ reverse primer

$12.1 \mathrm{ul} \mathrm{d \textrm {H } _ { 2 } \mathrm { O }}$

12.5 ul OneTaq Quick-Load 2X Master Mix (M0486L)

Rxn: $94^{\circ} \mathrm{C} 30 \mathrm{sec}$

$94^{\circ} \mathrm{C} 30 \mathrm{sec}$

$58^{\circ} \mathrm{C} 30 \mathrm{sec}$

34 cycles

$68^{\circ} \mathrm{C} 30 \mathrm{sec}$

$68^{\circ} \mathrm{C} 5$ minutes

$8^{\circ} \mathrm{C}$ Hold 
-The positive colonies will show $\sim 630$ bps amplicon.

- The positive colonies are incubated in $5 \mathrm{ml}$ of LB+Kanamycin and miiniprepped using Qiaprep Spin Miniprep kit (Qiagen 27106).

-Resulting DNA is sequenced using M13Reverse and intseq_forward primers (GTTCGATTCCCGGCCGATG)

Vector sequences:

pUC57_Kan_gw_OK

TCGCGCGTTTCGGTGATGACGGTGAAAACCTCTGACACATGCAGCTCCCGGAGACGGTCA CAGCTTGTCTGTAAGCGGATGCCGGGAGCAGACAAGCCCGTCAGGGCGCGTCAGCGGGT GTTGGCGGGTGTCGGGGCTGGCTTAACTATGCGGCATCAGAGCAGATTGTACTGAGAGTG CACCATATGCGGTGTGAAATACCGCACAGATGCGTAAGGAGAAAATACCGCATCAGGCGCC ATTCGCCATTCAGGCTGCGCAACTGTTGGGAAGGGCGATCGGTGCGGGCCTCTTCGCTATT ACGCCAGCTGGCGAAAGGGGGATGTGCTGCAAGGCGATTAAGTTGGGTAACGCCAGGGTT TTCCCAGTCACGACGTTGTAAAACGACGGCCAGTGAATTGACGCGTATTGG AAGCAGAGAGGGCGCCAGTGCTCACTACTTTTTATAATTCTCAACTTCTTTTTCCAGACTCA GTTCGTATATATAGACCTATTTTCAATTTAACGTCGTAGTACGATCATAACAACGGTTTTAGA GCTAGAAATAGCAAGTTAAAATAAGGCTAGTCCGTTATCAACTTGAAAAAGTGGCACCGAGT CGGTGCTTTTTTTGCGGCCGCCTCGAGCATG GTCATAGCTGTTTCCTGTGTGAAATTGTTAT CCGCTCACAATTCCACACAACATACGAGCCGGAAGCATAAAGTGTAAAGCCTGGGGTGCCT AATGAGTGAGCTAACTCACATTAATTGCGTTGCGCTCACTGCCCGCTTTCCAGTCGGGAAAC CTGTCGTGCCAGCTGCATTAATGAATCGGCCAACGCGCGGGGAGAGGCGGTTTGCGTATT GGGCGCTCTTCCGCTTCCTCGCTCACTGACTCGCTGCGCTCGGTCGTTCGGCTGCGGCGA GCGGTATCAGCTCACTCAAAGGCGGTAATACGGTTATCCACAGAATCAGGGGATAACGCAG GAAAGAACATGTGAGCAAAAGGCCAGCAAAAGGCCAGGAACCGTAAAAAGGCCGCGTTGC TGGCGTTTTTCCATAGGCTCCGCCCCCCTGACGAGCATCACAAAAATCGACGCTCAAGTCA GAGGTGGCGAAACCCGACAGGACTATAAAGATACCAGGCGTTTCCCCCTGGAAGCTCCCTC GTGCGCTCTCCTGTTCCGACCCTGCCGCTTACCGGATACCTGTCCGCCTTTCTCCCTTCGG GAAGCGTGGCGCTTTCTCATAGCTCACGCTGTAGGTATCTCAGTTCGGTGTAGGTCGTTCG CTCCAAGCTGGGCTGTGTGCACGAACCCCCCGTTCAGCCCGACCGCTGCGCCTTATCCGG TAACTATCGTCTTGAGTCCAACCCGGTAAGACACGACTTATCGCCACTGGCAGCAGCCACT GGTAACAGGATTAGCAGAGCGAGGTATGTAGGCGGTGCTACAGAGTTCTTGAAGTGGTGG CCTAACTACGGCTACACTAGAAGAACAGTATTTGGTATCTGCGCTCTGCTGAAGCCAGTTAC CTTCGGAAAAAGAGTTGGTAGCTCTTGATCCGGCAAACAAACCACCGCTGGTAGCGGTGGT TTTTTTGTTTGCAAGCAGCAGATTACGCGCAGAAAAAAAGGATCTCAAGAAGATCCTTTGAT CTTTTCTACGGGGTCTGACGCTCAGTGGAACGAAAACTCACGTTAAGGGATTTTGGTCATGA GATTATCAAAAAGGATCTTCACCTAGATCCTTTTAAATTAAAAATGAAGTTTTAAATCAATCTA AAGTATATATGAGTAAACTTGGTCTGACAGTTAGAAAAACTCATCGAGCATCAAATGAAACTG CAATTTATTCATATCAGGATTATCAATACCATATTTTTGAAAAAGCCGTTTCTGTAATGAAGGA GAAAACTCACCGAGGCAGTTCCATAGGATGGCAAGATCCTGGTATCGGTCTGCGATTCCGA CTCGTCCAACATCAATACAACCTATTAATTTCCCCTCGTCAAAAATAAGGTTATCAAGTGAGA AATCACCATGAGTGACGACTGAATCCGGTGAGAATGGCAAAAGTTTATGCATTTCTTTCCAG ACTTGTTCAACAGGCCAGCCATTACGCTCGTCATCAAAATCACTCGCATCAACCAAACCGTT ATTCATTCGTGATTGCGCCTGAGCGAGACGAAATACGCGATCGCTGTTAAAAGGACAATTAC AAACAGGAATCGAATGCAACCGGCGCAGGAACACTGCCAGCGCATCAACAATATTTTCACC TGAATCAGGATATTCTTCTAATACCTGGAATGCTGTTTTCCCAGGGATCGCAGTGGTGAGTA ACCATGCATCATCAGGAGTACGGATAAAATGCTTGATGGTCGGAAGAGGCATAAATTCCGT CAGCCAGTTTAGTCTGACCATCTCATCTGTAACATCATTGGCAACGCTACCTTTGCCATGTTT 


\begin{abstract}
CAGAAACAACTCTGGCGCATCGGGCTTCCCATACAATCGATAGATTGTCGCACCTGATTGC CCGACATTATCGCGAGCCCATTTATACCCATATAAATCAGCATCCATGTTGGAATTTAATCGC GGCCTAGAGCAAGACGTTTCCCGTTGAATATGGCTCATACTCTTCCTTTTTCAATATTATTGA AGCATTTATCAGGGTTATTGTCTCATGAGCGGATACATATTTGAATGTATTTAGAAAAATAAA CAAATAGGGGTTCCGCGCACATTTCCCCGAAAAGTGCCACCTGACGTCTAAGAAACCATTAT TATCATGACATTAACCTATAAAAATAGGCGTATCACGAGGCCCTTTCGTC
\end{abstract}

$\begin{array}{lrrrr}\text { sgRNA1_target } & \text { sgRNA1 } & \text { M13Frw primer } & \text { U6-3 promoter } & \text { sgRNA scaffold } \\ \text { M13rev primer Ori } & \text { KanR } & \text { bla promoter } & \text { underlined sequences are in }- \text { strand }\end{array}$

pUC57_Kan_gw_OK2

TCGCGCGTTTCGGTGATGACGGTGAAAACCTCTGACACATGCAGCTCCCGGAGACGGTCA CAGCTTGTCTGTAAGCGGATGCCGGGAGCAGACAAGCCCGTCAGGGCGCGTCAGCGGGT GTTGGCGGGTGTCGGGGCTGGCTTAACTATGCGGCATCAGAGCAGATTGTACTGAGAGTG CACCATATGCGGTGTGAAATACCGCACAGATGCGTAAGGAGAAAATACCGCATCAGGCGCC ATTCGCCATTCAGGCTGCGCAACTGTTGGGAAGGGCGATCGGTGCGGGCCTCTTCGCTATT ACGCCAGCTGGCGAAAGGGGGATGTGCTGCAAGGCGATTAAGTTGGGTAACGCCAGGGTT TTCCCAGTCACGACGTTGTAAAACGACGGCCAGTGAATTGACGCGTTTTTTTGCTCACCTGT GATTGCTCCTACTCAAATACAAAAACATCAAATTTTCTGTCAATAAAGCATATTTATTTATATT TATTTTACAGGAAAGAATTCCTTTTAAAGTGTATTTTAACCTATAATGAAAAACGATTAAAAAA AATACATAAAATAATTCGAAAATTTTTGAATAGCCCAGGTTGATAAAAATTCATTTCATACGTT TTATAACTTATGCCCCTAAGTATTTTTTGACCATAGTGTTTCAATTCTACATTAATTTTACAGA GTAGAATGAAACGCCACCTACTCAGCCAAGAGGCGAAAAGGTTAGCTCGCCAAGCAGAGA GGGCGCCAGTGCTCACTACTTTTTATAATTCTCAACTTCTTTTTCCAGACTCAGTTCGTATAT ATAGACCTATTTTCAATTTAACGTCGGGGCTTTGAGTGTGTGTAGACATCAAGCATCGGTGG TTCAGTGGTAGAATGCTCGCCTGCCACGCGGGCGGCCCGGGTTCGATTCCCGGCCGATGC AGTAGTACGATCATAACAACGGTTTTAGAGCTAGAAATAGCAAGTTAAAATAAGGCTAGTCC GTTATCAACTTGAAAAAGTGGCACCGAGTCGGTGC

\title{
AGAATAGTACCCTGCCACGGTACAGACCCGGGTTCGATAT
}

GCGGCCGCCTCGAGCATGGTCATAGCTGTTTCCTGTGTGAAATTGTTATCCGCTCACAAT TCCACACAACATACGAGCCGGAAGCATAAAGTGTAAAGCCTGGGGTGCCTAATGAGTGAGC TAACTCACATTAATTGCGTTGCGCTCACTGCCCGCTTTCCAGTCGGGAAACCTGTCGTGCCA GCTGCATTAATGAATCGGCCAACGCGCGGGGAGAGGCGGTTTGCGTATTGGGCGCTCTTC CGCTTCCTCGCTCACTGACTCGCTGCGCTCGGTCGTTCGGCTGCGGCGAGCGGTATCAGC TCACTCAAAGGCGGTAATACGGTTATCCACAGAATCAGGGGATAACGCAGGAAAGAACATG TGAGCAAAAGGCCAGCAAAAGGCCAGGAACCGTAAAAAGGCCGCGTTGCTGGCGTTTTTTCC ATAGGCTCCGCCCCCCTGACGAGCATCACAAAAATCGACGCTCAAGTCAGAGGTGGCGAAA CCCGACAGGACTATAAAGATACCAGGCGTTTCCCCCTGGAAGCTCCCTCGTGCGCTCTCCT GTTCCGACCCTGCCGCTTACCGGATACCTGTCCGCCTTTCTCCCTTCGGGAAGCGTGGCGC TTTCTCATAGCTCACGCTGTAGGTATCTCAGTTCGGTGTAGGTCGTTCGCTCCAAGCTGGG CTGTGTGCACGAACCCCCCGTTCAGCCCGACCGCTGCGCCTTATCCGGTAACTATCGTCTT GAGTCCAACCCGGTAAGACACGACTTATCGCCACTGGCAGCAGCCACTGGTAACAGGATTA GCAGAGCGAGGTATGTAGGCGGTGCTACAGAGTTCTTGAAGTGGTGGCCTAACTACGGCTA CACTAGAAGAACAGTATTTGGTATCTGCGCTCTGCTGAAGCCAGTTACCTTCGGAAAAAGAG TTGGTAGCTCTTGATCCGGCAAACAAACCACCGCTGGTAGCGGTGGTTTTTTTGTTTGCAAG CAGCAGATTACGCGCAGAAAAAAAGGATCTCAAGAAGATCCTTTGATCTTTTCTACGGGGTC TGACGCTCAGTGGAACGAAAACTCACGTTAAGGGATTTTGGTCATGAGATTATCAAAAAGGA TCTTCACCTAGATCCTTTTAAATTAAAAATGAAGTTTTAAATCAATCTAAAGTATATATGAGTA AACTTGGTCTGACAGTTAGAAAAACTCATCGAGCATCAAATGAAACTGCAATTTATTCATATC AGGATTATCAATACCATATTTTTGAAAAAGCCGTTTCTGTAATGAAGGAGAAAACTCACCGAG GCAGTTCCATAGGATGGCAAGATCCTGGTATCGGTCTGCGATTCCGACTCGTCCAACATCA ATACAACCTATTAATTTCCCCTCGTCAAAAATAAGGTTATCAAGTGAGAAATCACCATGAGTG ACGACTGAATCCGGTGAGAATGGCAAAAGTTTATGCATTTCTTTCCAGACTTGTTCAACAGG CCAGCCATTACGCTCGTCATCAAAATCACTCGCATCAACCAAACCGTTATTCATTCGTGATT 
bioRxiv preprint doi: https://doi.org/10.1101/2021.12.24.474112; this version posted December 24,2021 . The copyright holder for this preprint (which was not certified by peer review) is the author/funder, who has granted bioRxiv a license to display the preprint in perpetuity. It is made available under aCC-BY 4.0 International license.

\begin{abstract}
GCGCCTGAGCGAGACGAAATACGCGATCGCTGTTAAAAGGACAATTACAAACAGGAATCGA ATGCAACCGGCGCAGGAACACTGCCAGCGCATCAACAATATTTTCACCTGAATCAGGATATT CTTCTAATACCTGGAATGCTGTTTTCCCAGGGATCGCAGTGGTGAGTAACCATGCATCATCA GGAGTACGGATAAAATGCTTGATGGTCGGAAGAGGCATAAATTCCGTCAGCCAGTTTAGTC TGACCATCTCATCTGTAACATCATTGGCAACGCTACCTTTGCCATGTTTCAGAAACAACTCTG GCGCATCGGGCTTCCCATACAATCGATAGATTGTCGCACCTGATTGCCCGACATTATCGCG AGCCCATTTATACCCATATAAATCAGCATCCATGTTGGAATTTAATCGCGGCCTAGAGCAAG ACGTTTCCCGTTGAATATGGCTCATACTCTTCCTTTTTCAATATTATTGAAGCATTTATCAGG GTTATTGTCTCATGAGCGGATACATATTTGAATGTATTTAGAAAAATAAACAAATAGGGGTTC CGCGCACATTTCCCCGAAAAGTGCCACCTGACGTCTAAGAAACCATTATTATCATGACATTA ACCTATAAAAATAGGCGTATCACGAGGCCCTTTCGTC
\end{abstract}

Intseq_forward sgRNA1_target sgRNA1 U6-3promoter sgR̄NA scaffold underlined sequences are in -strand
M13Frw primer

KanR bla promoter

\title{
pM37_KozakGAL4
}

CTAAATTGTAAGCGTTAATATTTTGTTAAAATTCGCGTTAAATTTTTGTTAAATCAGCTCATTTT TTAACCAATAGGCCGAAATCGGCAAAATCCCTTATAAATCAAAAGAATAGACCGAGATAGGG TTGAGTGTTGTTCCAGTTTGGAACAAGAGTCCACTATTAAAGAACGTGGACTCCAACGTCAA AGGGCGAAAAACCGTCTATCAGGGCGATGGCCCACTACGTGAACCATCACCCTAATCAAGT TTTTTGGGGTCGAGGTGCCGTAAAGCACTAAATCGGAACCCTAAAGGGAGCCCCCGATTTA GAGCTTGACGGGGAAAGCCGGCGAACGTGGCGAGAAAGGAAGGGAAGAAAGCGAAAGGA GCGGGCGCTAGGGCGCTGGCAAGTGTAGCGGTCACGCTGCGCGTAACCACCACACCCGC CGCGCTTAATGCGCCGCTACAGGGCGCGTCCCATTCGCCATTCAGGCTGCGCAACTGTTG GGAAGGGCGATCGGTGCGGGCCTCTTCGCTATTACGCCAGCTGGCGAAAGGGGGATGTGC TGCAAGGCGATTAAGTTGGGTAACGCCAGGGTTTTCCCAGTCACGACGTTGTAAAACGACG GCCAGTGAGCGCGCGTAATACGACTCACTATAGGGCGAATTGGAGCTCCACCGCGGTGGC GTCTCTAAACGGTCTCTAAACGAAGACTTAAACCAAAATGAAGCTGCTGAGCAGCATCGAGC AGGCCTGCGATATCTGCCGCCTGAAGAAGCTGAAGTGCAGCAAGGAGAAGCCCAAGTGCG CCAAGTGCCTGAAGAACAACTGGGAGTGCCGCTACAGCCCCAAGACCAAGCGCAGCCCCC TGACCCGCGCCCACCTGACCGAGGTGGAGAGCCGCCTGGAGCGCCTGGAGCAGCTGTTC CTGCTGATCTTCCCCCGCGAGGATCTGGATATGATCCTGAAGATGGATAGCCTGCAGGATA TCAAGGCCCTGCTGACCGGCCTGTTCGTGCAGGATAACGTGAACAAGGATGCCGTGACCG ATCGCCTGGCCAGCGTGGAAACCGATATGCCCCTGACCCTGCGCCAGCACCGCATCAGCG CCACCAGCAGCAGCGAGGAGAGCAGCAACAAGGGCCAGCGCCAGCTGACCGTGAGCATC GATAGCGCCGCCCACCACGATAACAGCACCATCCCCCTGGATTTCATGCCCCGCGATGCC CTGCACGGCTTCGATTGGAGCGAGGAGGATGATATGAGCGATGGCCTGCCCTTCCTGAAAA CCGATCCCAACAACAACGGCTTCTTCGGCGATGGCAGCCTGCTGTGCATCCTGCGCAGCAT CGGCTTCAAGCCCGAGAACTACACCAACAGCAACGTGAACCGCCTGCCCACCATGATCACC GATCGCTACACCCTGGCCAGCCGCAGCACCACCAGCCGCCTGCTGCAGAGCTACCTGAAC AACTTCCACCCCTACTGCCCCATCGTGCACAGCCCCACCCTGATGATGCTGTACAACAACC AGATCGAGATCGCCAGCAAGGATCAGTGGCAGATCCTGTTCAACTGCATCCTGGCCATCGG CGCCTGGTGCATCGAGGGCGAGAGCACCGATATCGATGTGTTCTACTACCAGAACGCCAA GAGCCACCTGACCAGCAAGGTGTTCGAGAGCGGCAGCATCATCCTGGTGACCGCCCTGCA CCTGCTGAGCCGCTACACCCAGTGGCGCCAAAAGACCAACACCAGCTACAACTTCCACAGC TTCAGCATCCGCATGGCCATCAGCCTGGGCCTGAACCGCGATCTGCCCAGCAGCTTCAGC GATAGCAGCATCCTGGAGCAGCGCCGCCGCATCTGGTGGAGCGTGTACAGCTGGGAGATC CAGCTGAGCCTGCTGTACGGCCGCAGCATCCAGCTGAGCCAGAACACCATCAGCTTCCCC AGCAGCGTGGATGATGTGCAGCGCACCACCACCGGCCCCACCATCTACCACGGCATCATC GAGACTGCCCGCCTGCTGCAGGTGTTCACCAAGATCTACGAGCTGGATAAGACCGTGACC GCCGAGAAGAGCCCCATCTGCGCCAAGAAGTGCCTGATGATCTGCAACGAGATCGAGGAG GTGAGCCGCCAGGCCCCCAAGTTCCTGCAGATGGATATCAGCACCACCGCCCTGACCAAC CTGCTGAAGGAGCACCCCTGGCTGAGCTTCACCCGCTTCGAGCTGAAGTGGAAGCAGCTG 


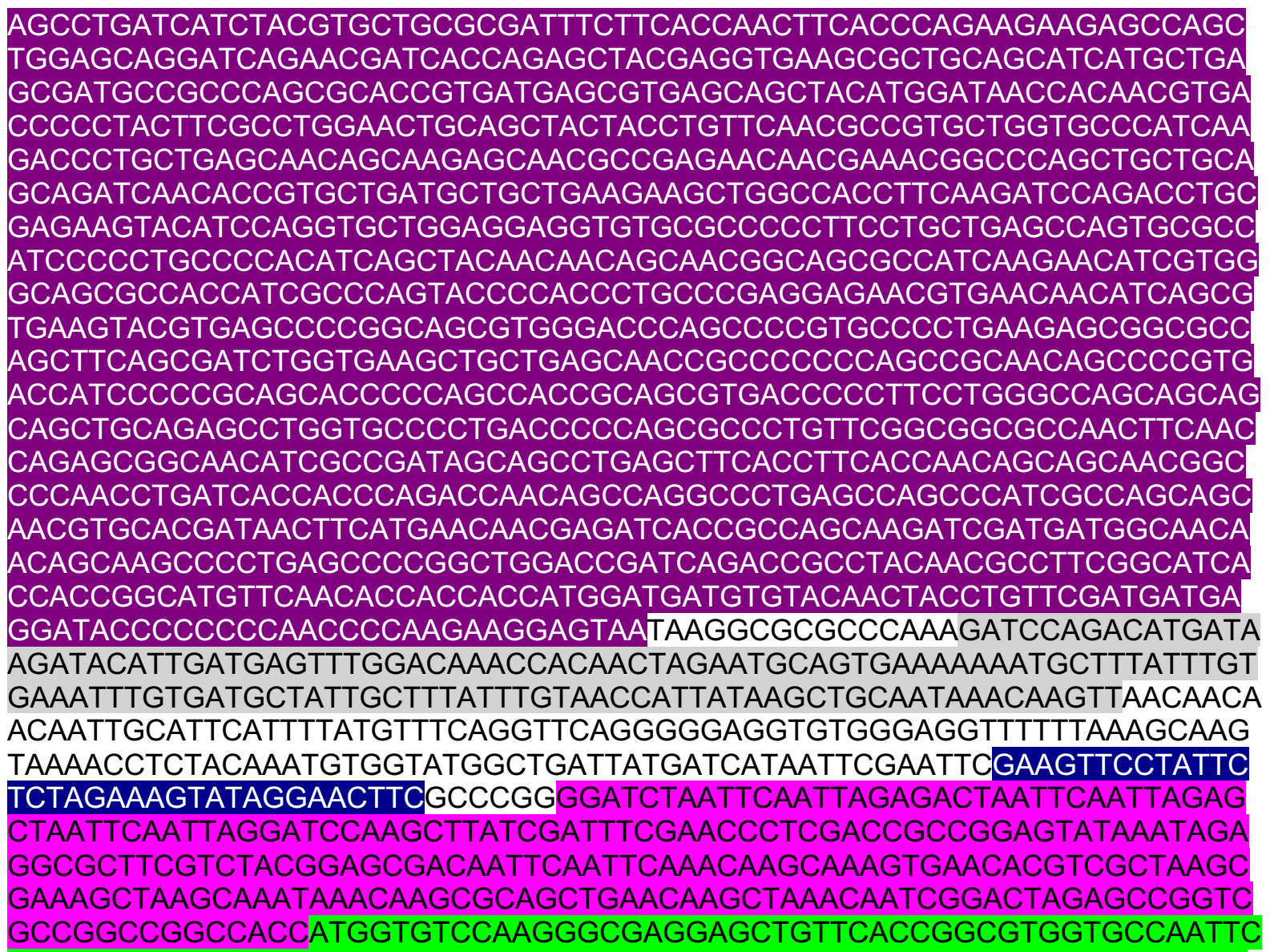
TGGTGGAGCTGGATGGCGACGTGAACGGCCACAAGTTCAGCGTGTCCGGCGAGGGCGAG GGCGACGCCACCTATGGAAAGCTGACCCTGAAGTTCATCTGCACCACCGGCAAGCTGCCC GTGCCATGGCCAACCCTCGTGACCACCCTGACCTATGGCGTGCAGTGCTTCAGCCGCTAC CCCGATCACATGAAGCAGCACGATTTCTTCAAGAGCGCCATGCCCGAGGGCTACGTGCAG GAGCGCACCATCTTTTTCAAGGATGACGGCAACTACAAGACCCGCGCCGAAGTGAAGTTCG AGGGCGATACCCTCGTGAACCGCATCGAGCTGAAGGGCATCGATTTCAAGGAGGATGGAA ACATCCTGGGCCACAAGCTGGAGTACAACTACAACAGCCACAACGTGTACATCATGGCCGA CAAGCAGAAGAACGGCATCAAGGCCAACTTCAAGATCCGCCACAACATCGAGGATGGCGG CGTGCAGCTGGCCGATCACTACCAGCAGAACACCCCAATCGGCGACGGCCCAGTGCTGCT GCCCGATAACCATTACCTGAGCACCCAGAGCGCCCTGAGCAAGGATCCCAACGAGAAGCG CGACCACATGGTGCTGCTGGAGTTTGTGACCGCCGCCGGCATTACCCTGGGCATGGATGA GCTGTACAAGTAGGATCCAGACATGATAAGATACATTGATGAGTTTGGACAAACCACAACTA GAATGCAGTGAAAAAAATGCTTTATTTGTGAAATTTGTGATGCTATTGCTTTATTTGTAACCAT TATAAGCTGCAATAAACAAGTTAACAACAACAATTGCATTCATTTTATGTTTCAGGTTCAGGG GGAGGTGTGGGAGGTTTTTTAAAGCAAGTAAAACCTCTACAAATGTGGTATGGCTGATTATG ATCAGAAGTTCCTATTCTCTAGAAAGTATAGGAACTTCTCGCGCTCGCGCGACTGACGGTC GTAAGCACCCGCGTACGTGCGCGTTGTCTTCCGCGTGAGACCCGCGTGAGACGGATATCA AGCTTATCGATACCGTCGACCTCGAGGGGGGGCCCGGTACCCAGCTTTTGTTCCCTTTAGT GAGGGTTAATTGCGCGCTTGGCGTAATCATGGTCATAGCTGTTTCCTGTGTGAAATTGTTAT CCGCTCACAATTCCACACAACATACGAGCCGGAAGCATAAAGTGTAAAGCCTGGGGTGCCT AATGAGTGAGCTAACTCACATTAATTGCGTTGCGCTCACTGCCCGCTTTCCAGTCGGGAAAC CTGTCGTGCCAGCTGCATTAATGAATCGGCCAACGCGCGGGGAGAGGCGGTTTGCGTATT GGGCGCTCTTCCGCTTCCTCGCTCACTGACTCGCTGCGCTCGGTCGTTCGGCTGCGGCGA GCGGTATCAGCTCACTCAAAGGCGGTAATACGGTTATCCACAGAATCAGGGGATAACGCAG GAAAGAACATGTGAGCAAAAGGCCAGCAAAAGGCCAGGAACCGTAAAAAGGCCGCGTTGC TGGCGTTTTTCCATAGGCTCCGCCCCCCTGACGAGCATCACAAAAATCGACGCTCAAGTCA GAGGTGGCGAAACCCGACAGGACTATAAAGATACCAGGCGTTTCCCCCTGGAAGCTCCCTC GTGCGCTCTCCTGTTCCGACCCTGCCGCTTACCGGATACCTGTCCGCCTTTCTCCCTTCGG GAAGCGTGGCGCTTTCTCATAGCTCACGCTGTAGGTATCTCAGTTCGGTGTAGGTCGTTCG 
bioRxiv preprint doi: https://doi.org/10.1101/2021.12.24.474112; this version posted December 24,2021 . The copyright holder for this preprint (which was not certified by peer review) is the author/funder, who has granted bioRxiv a license to display the preprint in perpetuity. It is made available under aCC-BY 4.0 International license.

CTCCAAGCTGGGCTGTGTGCACGAACCCCCCGTTCAGCCCGACCGCTGCGCCTTATCCGG TAACTATCGTCTTGAGTCCAACCCGGTAAGACACGACTTATCGCCACTGGCAGCAGCCACT GGTAACAGGATTAGCAGAGCGAGGTATGTAGGCGGTGCTACAGAGTTCTTGAAGTGGTGG CCTAACTACGGCTACACTAGAAGGACAGTATTTGGTATCTGCGCTCTGCTGAAGCCAGTTAC CTTCGGAAAAAGAGTTGGTAGCTCTTGATCCGGCAAACAAACCACCGCTGGTAGCGGTGGT TTTTTTGTTTGCAAGCAGCAGATTACGCGCAGAAAAAAAGGATCTCAAGAAGATCCTTTGAT CTTTTCTACGGGGTCTGACGCTCAGTGGAACGAAAACTCACGTTAAGGGATTTTGGTCATGA GATTATCAAAAAGGATCTTCACCTAGATCCTTTTAAATTAAAAATGAAGTTTTAAATCAATCTA AAGTATATATGAGTAAACTTGGTCTGACAGTTACCAATGCTTAATCAGTGAGGCACCTATCTC AGCGATCTGTCTATTTCGTTCATCCATAGTTGCCTGACTCCCCGTCGTGTAGATAACTACGA TACGGGAGGGCTTACCATCTGGCCCCAGTGCTGCAATGATACCGCGTGACCCACGCTCAC CGGCTCCAGATTTATCAGCAATAAACCAGCCAGCCGGAAGGGCCGAGCGCAGAAGTGGTC CTGCAACTTTATCCGCCTCCATCCAGTCTATTAATTGTTGCCGGGAAGCTAGAGTAAGTAGT TCGCCAGTTAATAGTTTGCGCAACGTTGTTGCCATTGCTACAGGCATCGTGGTGTCACGCTC GTCGTTTGGTATGGCTTCATTCAGCTCCGGTTCCCAACGATCAAGGCGAGTTACATGATCCC CCATGTTGTGCAAAAAAGCGGTTAGCTCCTTCGGTCCTCCGATCGTTGTCAGAAGTAAGTTG GCCGCAGTGTTATCACTCATGGTTATGGCAGCACTGCATAATTCTCTTACTGTCATGCCATC CGTAAGATGCTTTTCTGTGACTGGTGAGTACTCAACCAAGTCATTCTGAGAATAGTGTATGC GGCGACCGAGTTGCTCTTGCCCGGCGTCAATACGGGATAATACCGCGCCACATAGCAGAA CTTTAAAAGTGCTCATCATTGGAAAACGTTCTTCGGGGCGAAAACTCTCAAGGATCTTACCG CTGTTGAGATCCAGTTCGATGTAACCCACTCGTGCACCCAACTGATCTTCAGCATCTTTTAC ITTCACCAGCGTTTCTGGGTGAGCAAAAACAGGAAGGCAAAATGCCGCAAAAAAGGGAATA AGGGCGACACGGAAATGTTGAATACTCATACTCTTCCTTTTTCAATATTATTGAAGCATTTAT CAGGGTTATTGTCTCATGAGCGGATACATATTTGAATGTATTTAGAAAAATAAACAAATAGGG GTTCCGCGCACATTTCCCCGAAAAGTGCCAC

\section{FRT SV40polyA GFP 3XP3-Hs70 promoter M13Frw primer KozakGAL4 AmpR}

pM37_SA_KozakGAL4

CTAAATTGTAAGCGTTAATATTTTGTTAAAATTCGCGTTAAATTTTTGTTAAATCAGCTCATTTT TTAACCAATAGGCCGAAATCGGCAAAATCCCTTATAAATCAAAAGAATAGACCGAGATAGGG TTGAGTGTTGTTCCAGTTTGGAACAAGAGTCCACTATTAAAGAACGTGGACTCCAACGTCAA AGGGCGAAAAACCGTCTATCAGGGCGATGGCCCACTACGTGAACCATCACCCTAATCAAGT TTTTTGGGGTCGAGGTGCCGTAAAGCACTAAATCGGAACCCTAAAGGGAGCCCCCGATTTA GAGCTTGACGGGGAAAGCCGGCGAACGTGGCGAGAAAGGAAGGGAAGAAAGCGAAAGGA GCGGGCGCTAGGGCGCTGGCAAGTGTAGCGGTCACGCTGCGCGTAACCACCACACCCGC CGCGCTTAATGCGCCGCTACAGGGCGCGTCCCATTCGCCATTCAGGCTGCGCAACTGTTG GGAAGGGCGATCGGTGCGGGCCTCTTCGCTATTACGCCAGCTGGCGAAAGGGGGATGTGC TGCAAGGCGATTAAGTTGGGTAACGCCAGGGTTTTCCCAGTCACGACGTTGTAAAACGACG GCCAGTGAGCGCGCGTAATACGACTCACTATAGGGCGAATTGGAGCTCCACCGCGGTGGC GTCTCTAAACGGTCTCTAAACGAAGACTTAAACAGTCGATCCAACATGGCGACTTGTCCCAT CCCCGGCATGTTTAAATATACTAATTATTCTTGAACTAATTTTAATCAACCGATTTATCTCTCT TCCGCAGCCAAAATGAAGCTGCTGAGCAGCATCGAGCAGGCCTGCGATATCTGCCGCCTG AAGAAGCTGAAGTGCAGCAAGGAGAAGCCCAAGTGCGCCAAGTGCCTGAAGAACAACTGG GAGTGCCGCTACAGCCCCAAGACCAAGCGCAGCCCCCTGACCCGCGCCCACCTGACCGA GGTGGAGAGCCGCCTGGAGCGCCTGGAGCAGCTGTTCCTGCTGATCTTCCCCCGCGAGGA TCTGGATATGATCCTGAAGATGGATAGCCTGCAGGATATCAAGGCCCTGCTGACCGGCCTG TTCGTGCAGGATAACGTGAACAAGGATGCCGTGACCGATCGCCTGGCCAGCGTGGAAACC GATATGCCCCTGACCCTGCGCCAGCACCGCATCAGCGCCACCAGCAGCAGCGAGGAGAGC AGCAACAAGGGCCAGCGCCAGCTGACCGTGAGCATCGATAGCGCCGCCCACCACGATAAC AGCACCATCCCCCTGGATTTCATGCCCCGCGATGCCCTGCACGGCTTCGATTGGAGCGAG 
bioRxiv preprint doi: https://doi.org/10.1101/2021.12.24.474112; this version posted December 24, 2021. The copyright holder for this preprint (which was not certified by peer review) is the author/funder, who has granted bioRxiv a license to display the preprint in perpetuity. It is made available under aCC-BY 4.0 International license.

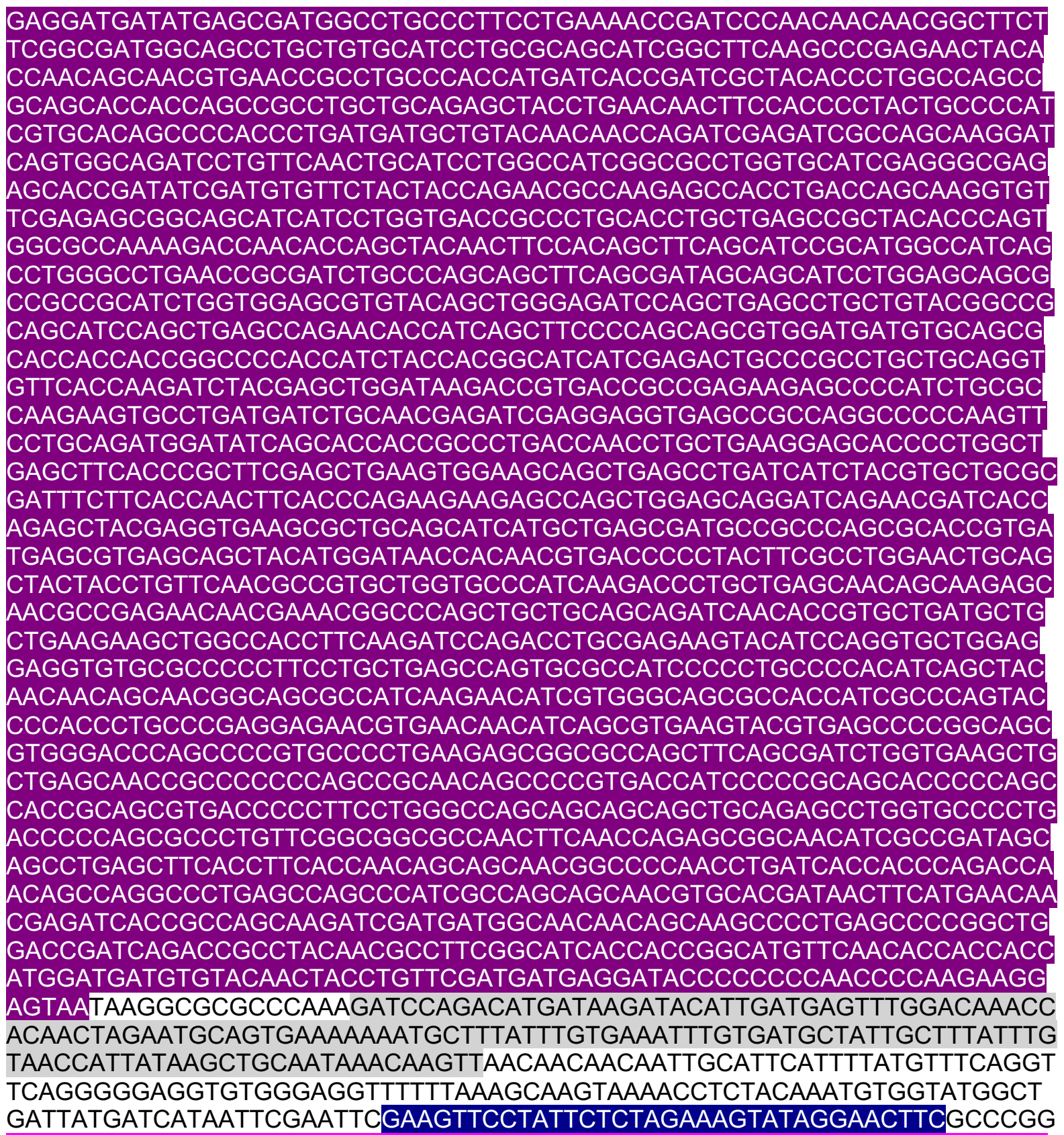
GGATCTAATTCAATTAGAGACTAATTCAATTAGAGCTAATTCAATTAGGATCCAAGCTTATCG ATTTCGAACCCTCGACCGCCGGAGTATAAATAGAGGCGCTTCGTCTACGGAGCGACAATTC AATTCAAACAAGCAAAGTGAACACGTCGCTAAGCGAAAGCTAAGCAAATAAACAAGCGCAG CTGAACAAGCTAAACAATCGGACTAGAGCCGGTCGCCGGCCGGCCACCATGGTGTCCAAG GGCGAGGAGCTGTTCACCGGCGTGGTGCCAATTCTGGTGGAGCTGGATGGCGACGTGAAC GGCCACAAGTTCAGCGTGTCCGGCGAGGGCGAGGGCGACGCCACCTATGGAAAGCTGAC CCTGAAGTTCATCTGCACCACCGGCAAGCTGCCCGTGCCATGGCCAACCCTCGTGACCAC CCTGACCTATGGCGTGCAGTGCTTCAGCCGCTACCCCGATCACATGAAGCAGCACGATTTC TTCAAGAGCGCCATGCCCGAGGGCTACGTGCAGGAGCGCACCATCTTTTTCAAGGATGACG GCAACTACAAGACCCGCGCCGAAGTGAAGTTCGAGGGCGATACCCTCGTGAACCGCATCG AGCTGAAGGGCATCGATTTCAAGGAGGATGGAAACATCCTGGGCCACAAGCTGGAGTACAA CTACAACAGCCACAACGTGTACATCATGGCCGACAAGCAGAAGAACGGCATCAAGGCCAAC TTCAAGATCCGCCACAACATCGAGGATGGCGGCGTGCAGCTGGCCGATCACTACCAGCAG AACACCCCAATCGGCGACGGCCCAGTGCTGCTGCCCGATAACCATTACCTGAGCACCCAG AGCGCCCTGAGCAAGGATCCCAACGAGAAGCGCGACCACATGGTGCTGCTGGAGTTTGTG ACCGCCGCCGGCATTACCCTGGGCATGGATGAGCTGTACAAGTAGGATCCAGACATGATAA GATACATTGATGAGTTTGGACAAACCACAACTAGAATGCAGTGAAAAAAATGCTTTATTTGTG 


\begin{abstract}
AAATTTGTGATGCTATTGCTTTATTTGTAACCATTATAAGCTGCAATAAACAAGTTAACAACAA CAATTGCATTCATTTTATGTTTCAGGTTCAGGGGGAGGTGTGGGAGGTTTTTTAAAGCAAGT AAAACCTCTACAAATGTGGTATGGCTGATTATGATCAGAAGTTCCTATTCTCTAGAAAGTATA GGAACTTCTCGCGCTCGCGCGACTGACGGTCGTAAGCACCCGCGTACGTGCGCGTTGTCT TCCGCGTGAGACCCGCGTGAGACGGATATCAAGCTTATCGATACCGTCGACCTCGAGGGG GGGCCCGGTACCCAGCTTTTGTTCCCTTTAGTGAGGGTTAATTGCGCGCTTGGCGTAATCA TGGTCATAGCTGTTTCCTGTGTGAAATTGTTATCCGCTCACAATTCCACACAACATACGAGC CGGAAGCATAAAGTGTAAAGCCTGGGGTGCCTAATGAGTGAGCTAACTCACATTAATTGCG TTGCGCTCACTGCCCGCTTTCCAGTCGGGAAACCTGTCGTGCCAGCTGCATTAATGAATCG GCCAACGCGCGGGGAGAGGCGGTTTGCGTATTGGGCGCTCTTCCGCTTCCTCGCTCACTG ACTCGCTGCGCTCGGTCGTTCGGCTGCGGCGAGCGGTATCAGCTCACTCAAAGGCGGTAA TACGGTTATCCACAGAATCAGGGGATAACGCAGGAAAGAACATGTGAGCAAAAGGCCAGCA AAAGGCCAGGAACCGTAAAAAGGCCGCGTTGCTGGCGTTTTTCCATAGGCTCCGCCCCCCT GACGAGCATCACAAAAATCGACGCTCAAGTCAGAGGTGGCGAAACCCGACAGGACTATAAA GATACCAGGCGTTTCCCCCTGGAAGCTCCCTCGTGCGCTCTCCTGTTCCGACCCTGCCGCT TACCGGATACCTGTCCGCCTTTCTCCCTTCGGGAAGCGTGGCGCTTTCTCATAGCTCACGC TGTAGGTATCTCAGTTCGGTGTAGGTCGTTCGCTCCAAGCTGGGCTGTGTGCACGAACCCC CCGTTCAGCCCGACCGCTGCGCCTTATCCGGTAACTATCGTCTTGAGTCCAACCCGGTAAG ACACGACTTATCGCCACTGGCAGCAGCCACTGGTAACAGGATTAGCAGAGCGAGGTATGTA GGCGGTGCTACAGAGTTCTTGAAGTGGTGGCCTAACTACGGCTACACTAGAAGGACAGTAT TTGGTATCTGCGCTCTGCTGAAGCCAGTTACCTTCGGAAAAAGAGTTGGTAGCTCTTGATCC GGCAAACAAACCACCGCTGGTAGCGGTGGTTTTTTTGTTTGCAAGCAGCAGATTACGCGCA GAAAAAAAGGATCTCAAGAAGATCCTTTGATCTTTTCTACGGGGTCTGACGCTCAGTGGAAC GAAAACTCACGTTAAGGGATTTTGGTCATGAGATTATCAAAAAGGATCTTCACCTAGATCCTT TTAAATTAAAAATGAAGTTTTAAATCAATCTAAAGTATATATGAGTAAACTTGGTCTGACAGTT ACCAATGCTTAATCAGTGAGGCACCTATCTCAGCGATCTGTCTATTTCGTTCATCCATAGTTG CCTGACTCCCCGTCGTGTAGATAACTACGATACGGGAGGGCTTACCATCTGGCCCCAGTGC TGCAATGATACCGCGTGACCCACGCTCACCGGCTCCAGATTTATCAGCAATAAACCAGCCA GCCGGAAGGGCCGAGCGCAGAAGTGGTCCTGCAACTTTATCCGCCTCCATCCAGTCTATTA ATTGTTGCCGGGAAGCTAGAGTAAGTAGTTCGCCAGTTAATAGTTTGCGCAACGTTGTTGCC ATTGCTACAGGCATCGTGGTGTCACGCTCGTCGTTTGGTATGGCTTCATTCAGCTCCGGTTC CCAACGATCAAGGCGAGTTACATGATCCCCCATGTTGTGCAAAAAAGCGGTTAGCTCCTTC GGTCCTCCGATCGTTGTCAGAAGTAAGTTGGCCGCAGTGTTATCACTCATGGTTATGGCAG CACTGCATAATTCTCTTACTGTCATGCCATCCGTAAGATGCTTTTCTGTGACTGGTGAGTACT CAACCAAGTCATTCTGAGAATAGTGTATGCGGCGACCGAGTTGCTCTTGCCCGGCGTCAAT ACGGGATAATACCGCGCCACATAGCAGAACTTTAAAAGTGCTCATCATTGGAAAACGTTCTT CGGGGCGAAAACTCTCAAGGATCTTACCGCTGTTGAGATCCAGTTCGATGTAACCCACTCG TGCACCCAACTGATCTTCAGCATCTTTTACTTTCACCAGCGTTTCTGGGTGAGCAAAAACAG GAAGGCAAAATGCCGCAAAAAAGGGAATAAGGGCGACACGGAAATGTTGAATACTCATACT CTTCCTTTTTCAATATTATTGAAGCATTTATCAGGGTTATTGTCTCATGAGCGGATACATATTT GAATGTATTTAGAAAAATAAACAAATAGGGGTTCCGCGCACATTTCCCCGAAAAGTGCCAC
\end{abstract}

